\title{
Evolution of interstellar dust and stardust in the solar neighbourhood ${ }^{\star}$
}

\author{
S. Zhukovska ${ }^{1}$, H.-P. Gail ${ }^{1}$, and M. Trieloff ${ }^{2}$ \\ ${ }^{1}$ Center for Astronomy, Institute for Theoretical Astrophysics, University of Heidelberg, Albert-Überle-Str. 2, 69120 Heidelberg, \\ Germany \\ e-mail: gail@ita.uni-heidelberg.de \\ ${ }^{2}$ Mineralogical Institute, University of Heidelberg, Im Neuenheimer Feld 236, 69121 Heidelberg, Germany
}

Received 4 May 2007 / Accepted 25 September 2007

\begin{abstract}
Aims. We studied the evolution of the abundance in interstellar dust species that originate in stellar sources and from condensation in molecular clouds in the local interstellar medium of the Milky Way. We determined from this the input of dust material to the Solar System.

Methods. A one-zone chemical evolution model of the Milky Way for the elemental composition of the disk combined with an evolution model for its interstellar dust component similar to that of Dwek (1998) is developed. The dust model considers dust-mass return from AGB stars as calculated from synthetic AGB models combined with models for dust condensation in stellar outflows. Supernova dust formation is included in a simple parametrised form that is gauged by observed abundances of presolar dust grains with a supernova origin. For dust growth in the ISM, a simple method is developed for coupling this with disk and dust evolution models.

Results. A chemical evolution model of the solar neighbourhood in the Milky Way is calculated, which forms the basis for calculating a model of the evolution of the interstellar dust population at the galactocentric radius of the Milky Way. The model successfully passes all standard tests for the reliability of such models. In particular the abundance evolution of the important dust-forming elements is compared with observational results for the metallicity-dependent evolution of the abundances for G-type stars from the solar neighbourhood. It is found that the new tables of Nomoto et al. (2006) for the heavy element production give much better results for the abundance evolution of these important elements than the widely used tables of Woosley \& Weaver (1995). The time evolution for the abundance of the following dust species is followed in the model: silicate, carbon, silicon carbide, and iron dust from AGB stars and from supernovae, as well as silicate, carbon, and iron dust grown in molecular clouds. It is shown that the interstellar dust population is dominated by dust accreted in molecular clouds; stardust only forms a minor fraction. Most of the dust material entering the Solar System at its formation does not show isotopic abundance anomalies of the refractory elements, i.e., inconspicuous isotopic abundances do not point to a Solar System origin for dust grains. The observed abundance ratios of presolar dust grains formed in supernova ejecta and in AGB star outflows requires that, for the ejecta from supernovae, the fraction of refractory elements condensed into dust is 0.15 for carbon dust and is quite small $\left(\sim 10^{-4}\right)$ for other dust species.
\end{abstract}

Key words. ISM: abundances - ISM: dust, extinction - galaxies: ISM - Galaxy: evolution

\section{Introduction}

In this paper we intend to study the population of dust grains in the interstellar medium of the Milky Way, its composition and evolution, and the input of these grains into the Solar System. Part of these grains are formed in stellar ejecta or stellar winds of highly evolved stars from the refractory elements therein and the resulting gas-dust mixture is ultimately mixed with the general interstellar matter. But generally only some fraction of the refractory elements in the ejecta is really condensed into solid phases; a big mass fraction of the refractory elements returned to the ISM - or sometimes even most of it - stays in the gas phase. Turbulent mixing in the ISM rapidly intermingles the material that is ejected by the many different stellar sources. While all the dust particles from stellar sources, called stardust particles, retain their peculiar isotopic compositions of a number of elements indicative of their formation sites, in the gas phase the material from all sources is mixed together and the resulting

\footnotetext{
* Appendix A is only available in electronic form at http: //www . aanda.org
}

isotopic composition of the mix is different from that of the stardust particles. The refractory elements in the ISM gas therefore have different isotopic compositions than the same elements found in stardust grains.

Stardust grains are found in the Solar System as a rare fraction of the fine grained matrix material of meteorites (e.g. Bernatowicz \& Zinner 1997; Hoppe 2004; Nguyen et al. 2007). They are identified as such by the unusual isotopic composition of at least one element that shows that the grains have condensed from material that contains freshly synthesised nuclei from stellar burning zones. Laboratory studies have found a wide assortment of such stardust grains, also called presolar dust particles, with a variety of chemical compositions that can be associated with a variety of stellar sources. The composition of the grain material indicates two basically different chemical environments of formation, (1) a carbon-rich environment, and (2) an oxygenrich environment, which yield two completely different groups of mineral compounds:

1. solid graphitic carbon, diamond, silicon carbide, silicon nitride, and 
Table 1. Comparison of some observed properties of the galactic disk in the solar neighbourhood with model results.

\begin{tabular}{llll}
\hline \hline Observable & Model & Observed & Reference \\
\hline Total surface density $\Sigma_{\text {tot }}\left[M_{\odot} \mathrm{pc}^{-2}\right]$ & 56 & $50-62$ & Holmberg \& Flynn (2004) \\
ISM surface density $\Sigma_{\text {ISM }}\left[M_{\odot} \mathrm{pc}^{-2}\right]$ & 9.7 & $7-13$ & Dickey (1993) \\
& & $13-14$ & Olling \& Merrifield (2001) \\
Gas fraction $\Sigma_{\text {ISM }} / \Sigma_{\text {tot }}$ & 0.17 & $0.05-0.2$ & \\
Surface density of visible stars $\Sigma_{*}\left[M_{\odot} \mathrm{pc}^{-2}\right]$ & 38.6 & $30-40$ & Gilmore et al. (1989) \\
Surface density of stellar remnants $\left[M_{\odot} \mathrm{pc}^{-2}\right]$ & 7.7 & $2-4$ & Mera et al. (1998) \\
Star formation rate $B\left[M_{\odot} \mathrm{pc}^{-2} \mathrm{Gyr}^{-1}\right]$ & 3.1 & $3.5-5$ & Rana 1991 \\
SN II rate $R_{\mathrm{SNII}}\left[\mathrm{pc}^{-2} \mathrm{Gyr}^{-1}\right]$ & 0.016 & $0.009-0.0326$ & Tammann et al. (1994) \\
SN Ia rate $R_{\mathrm{SNIa}}\left[\mathrm{pc}^{-2} \mathrm{Gyr}^{-1}\right]$ & 0.0024 & $0.0015-0.0109$ & Tammann et al. (1994) \\
Infall rate $\left[M_{\odot} \mathrm{pc}^{-2} \mathrm{Gyr}^{-1}\right]$ & 1.45 & $0.5-5$ & Braun \& Thilker (2004) \\
\hline
\end{tabular}

2. corundum, hibonite, spinel, magnesium-iron-silicates.

Besides of these main components, a number of minor components (e.g. titanium oxide, solid solutions of titanium carbide with zirconium and molybdenum carbide, kamacite, and cohenite) have been identified that are so far only known to exist as inclusions in grains of the major dust components. Additional components may exist and await identification, in particular, since some kinds of dust may not survive all the stages between the stellar source and the final laboratory investigation. The observed isotopic anomaliesessentially indicate two different kinds of stellar sources of the presolar dust grains, (1) AGB stars and (2) core-collapse supernovae.

From the instant of the formation in stellar ejecta on, stardust grains are subject to destructive processes in the ISM by sputtering and shattering processes induced by supernova (SN) shocks (cf. Jones et al. 1996, and references therein). They are finally incorporated into newly formed stars and their planetary systems after about 2.5 Gyr residence in the ISM, which is also the typical timescale for replenishment of the ISM with new stardust. Theoretical studies have shown that typical lifetimes against destruction by SN shocks are only about 0.5 Gyr (Jones et al. 1996). This rather short timescale compared to the timescale for replenishment would result in very low dust abundance in the ISM. This, however, is not observed. Instead one observes a high degree of depletion of the refractory elements in the gas phase of the ISM (e.g. Savage \& Sembach 1996; Jenkins 2004), and this clearly requires additional growth processes in the interstellar medium that tie up the atoms of the refractory elements in dust. The only possible sites where accretion of gas phase material onto grains may proceed with a reasonably short timescale are the dense molecular clouds of the ISM (Draine 1990). Any solid phase material grown in molecular clouds, the $M C$-grown $d u s t$, has isotopic compositions of the refractory elements that are different from what is found in the stardust particles and may be identified by this property. Such dust material may be found both as coating of stardust grains and as separate grains.

Unfortunately, the isotopic composition of the refractory elements in MC-grown dust grains incorporated into the Solar System equals the isotopic composition of the elements in the Solar System, which makes it impossible to distinguish by laboratory investigations of isotopic abundances of refractory elements alone between dust formed in the Solar System and MC-grown dust. There are other indications, however, that point to a presolar origin of some fraction of the interplanetary dust particles, the GEMS (Bradley 2003; Messenger et al. 2003), which shows isotopic abundances of refractory elements corresponding to normal Solar System isotopic abundances and which therefore are likely to be MC-grown dust grains. The category of presolar dust grains therefore also includes the MC-grown dust species that are isotopically inconspicuous, a property presently making them difficult to be identified as of extrasolar origin.

If we intend to calculate the abundances for the different components of the interstellar dust mixture in the Milky Way at the solar circle and in particular the composition of the dust mixture from which the Solar System formed, we have to construct a model of the Milky Way's chemical evolution that is coupled with a model for the evolution of the dust component of the interstellar matter. The evolution of the dust component is not independent of the evolution of the element abundances, since the refractory elements forming the dust are only gradually formed during the course of the chemical evolution of the galaxy. The model for the dust evolution needs to consider the injection of stardust into the ISM, the destruction processes of dust in the ISM, and the growth processes in molecular clouds. Very simple models for the evolution of the dust content of galaxies have already been constructed (e.g. Lisenfeld \& Ferrara 1998; Hirashita 2000; Edmunds 2001; Morgan \& Edmunds 2003; Inoue 2003), but these are too simplistic to allow for a detailed calculation of the composition of the interstellar dust mixture. Only the method developed by Dwek (1998) integrating the chemical evolution of the galactic disk and the dust evolution into a common model is sufficiently general to allow a modelling of the complex interplay between the processes determining the dust evolution and has the potential of being extended to even more complex systems. This model is a one-zone model, i.e., the galactic disk is approximated by a set of independent cylinders with all physical variables within a cylinder averaged over the vertical direction with respect to the disks midplane; and a one-phase model, i.e., one averages the properties of the ISM over its different phases (cold, warm, and hot; cf. Tielens 2005). This type of model allows a successful, and at the same time, rather easy calculation of some important properties of the Milky Way disk, in particular of its chemical evolution (cf. Matteuchi 2003). The price one has to pay for the simplifications is that some processes, in particular those depending critically on the phase structure of the ISM, cannot be treated with sufficient accuracy. Nevertheless, the results obtained by Dwek (1998) show that such a simple model can be used successfully to calculate the evolution of the interstellar dust. We take the model of Dwek as a basis for constructing a model that allows a more complex mixture of stardust and MC-grown dust to be treated.

For the input of stardust from AGB-stars, we use our recent results for the dust production by AGB-stars (Ferrarotti \& Gail 2006), which are somewhat extended. These tables present rather detailed information on the amount and composition of stardust formed by AGB-stars. For stardust from SN, we follow the procedure of Dwek (1998) and use a simple parametrisation 
for the dust production rate since no other suitable information on dust production is available. Observations are inconclusive and the theory is only in its infancy (Schneider et al. 2004; Nozawa et al. 2003, and references therein). We, in turn, try to gain some insight into the dust production efficiency of supernovae by comparing our model results with the meteoritic abundances of stardust from SNe. The dust growth in molecular clouds is treated in more detail as in the model of Dwek (1998), since we intend to distinguish in the model between stardust and $\mathrm{MC}$-grown dust. In principle it would also be necessary to consider that, according to observations of element depletion in the ISM, the MC-grown dust has a definite core mantle structure with a more resilient core and a more easily destructible mantle. A theoretical treatment of this grain structure would require considering at least a two-phase interstellar medium (cf. Tielens 1998; Inoue 2003) and not a simple one-phase model as in our present calculation.

The plan of our paper is as follows. In Sect. 2 our evolution model for the solar neighbourhood of the Milky Way is introduced and some results for the chemical evolution discussed. In Sect. 3 the model for the dust return by stars is explained. Section 4 discusses the dust destruction and growth processes in the ISM, and Sect. 5 presents the results for the evolution of the interstellar dust. Some concluding remarks are given in Sect. 6.

\section{Chemical evolution}

To study the evolution of the dust content of our Galaxy we developed a standard open model of galactic chemical evolution. In this model the Milky Way is formed by the slow infall of primordial gas from the halo or intergalactic space. Merging with other galaxies seems not to have played a mayor role during most of the lifetime of the Milky Way, except for the very first evolutionary phase for which stellar dynamics (cf. Helmi et al. 2006) and elemental abundances (Reddy et al. 2006; Ramírez et al. 2007) indicate that there were major merging events. Merging is not considered in the model. In the one-zone approximation we neglect radial motions in the galactic disk (but cf. Vorobyov \& Shchekinov 2006) and consider its evolution in a set of independent rings.

We numerically solve a classical set of non-linear integrodifferential equations for the chemical evolution of the Milky Way following a mathematical formulation similar to Dwek (1998), who first extended the standard system of equations for the chemical evolution of the galactic disk (cf. Matteucci 2003) to include the evolution of the dust component of the Galaxy. The basic set of equations for the surface densities of gas, stars, nuclei, etc., is not repeated here but can be found in the papers cited before. We specify in the following only our choices for some important input quantities for the model calculations.

\subsection{Basic model parameters}

\subsubsection{Infall}

First models of chemical evolution of the Galaxy were simple "closed-box" models, in which the Galaxy's mass is already fixed at the initial instant of evolution. However, these models failed to reproduce the metallicity distribution of metalpoor stars, one of the most important observational constraints on chemical evolution modelling, a problem known as G-dwarf problem. Open models assume formation of the Galaxy by accretion of primordial or metal-poor gas from extragalactic sources to solve the G-dwarf problem, as was first suggested by Chiosi (1980) and later discussed by Pagel (1997). In open models the total surface density of the disk changes by the accretion of gas, outflows, and radial motions within the disk. For the Milky Way, outflows can be neglected due to the strong gravitational potential, and radial motions are neglected in the one-zone approximation. In our model the infall rate entirely defines the evolution of the total surface density.

Several scenarios for gas accretion have been proposed by different authors, suggesting different rates and sequences for formation of the galactic components, see Matteucci (2003) for details. Models assuming an exponentially decreasing infall of the gas are most successful in reproducing the G-dwarf distribution. Following Chiappini et al. (1997) we adopt a two-infall, exponentially decreasing model that assumes two subsequent episodes of Galaxy formation. Initially, the halo and thick disk are formed during a short period of about $\tau_{\mathrm{H}} \approx 1 \mathrm{Gyr}$, then the thin disk is formed by accretion of material on a much longer timescale of $\tau_{\mathrm{D}} \approx 7 \mathrm{Gyr}$ at Solar galactocentric radius (here $\left.r_{\odot}=8.5 \mathrm{kpc}\right)$. The accretion rate is given in this model by the expression:

$$
\begin{aligned}
& \frac{\mathrm{d} \Sigma_{i}(r, t)_{\mathrm{inf}}}{\mathrm{d} t}= \\
& \begin{cases}\left(X_{i}\right)_{\mathrm{inf}} A(r) \mathrm{e}^{-t / \tau_{\mathrm{H}}} & \text { for } t<t_{\text {thin }} \\
\left(X_{i}\right)_{\text {inf }} A(r) \mathrm{e}^{-t / \tau_{\mathrm{H}}}+\left(X_{i}\right)_{\mathrm{inf}}^{\prime} B(r) \mathrm{e}^{-\left(t-t_{\text {thin }}\right) / \tau_{\mathrm{D}}} & \text { for } t>t_{\text {thin }}\end{cases}
\end{aligned}
$$

where $t_{\text {thin }}=1 \mathrm{Gyr}$ is the time of onset of accretion onto the thin disk. The formation of the disk is assumed to start $t_{\mathrm{G}}=$ 13 Gyr ago. Both $\left(X_{i}\right)_{\text {inf }}$ and $\left(X_{i}\right)_{\text {inf }}^{\prime}$ denote element abundances of infalling gas, which we assume to be primordial.

The coefficients $A(r)$ and $B(r)$ are derived so as to reproduce the present-day density distribution of the disk. At the solar circle one has

$$
\begin{aligned}
& A\left(r_{\odot}\right)=\frac{\Sigma_{\mathrm{H}}\left(r_{\odot}, t_{\mathrm{G}}\right)}{\tau_{\mathrm{H}}\left(1-\mathrm{e}^{-t_{\mathrm{G}} / \tau_{\mathrm{H}}}\right)} \\
& B\left(r_{\odot}\right)=\frac{\Sigma_{\mathrm{tot}}\left(r_{\odot}, t_{\mathrm{G}}\right)-\Sigma_{\mathrm{H}}\left(r_{\odot}, t_{\mathrm{G}}\right)}{\tau_{\mathrm{D}}\left(1-\mathrm{e}^{-\left(t_{\mathrm{G}}-t_{\mathrm{thin}}\right) / \tau_{\mathrm{D}}}\right)} .
\end{aligned}
$$

For details we refer to Chiappini et al. (1997) and Alibes et al. (2001). The value $\Sigma_{\text {tot }}\left(r_{\odot}, t_{\mathrm{G}}\right)$ for the current total density of the disk is taken to be $56 M_{\odot} \mathrm{pc}^{-2}$ according to Holmberg \& Flynn (2004). For the contribution of the thick disk to the total surface density, we choose $\Sigma_{\mathrm{H}}=10 \mathrm{M}_{\odot} \mathrm{pc}^{-2}$.

\subsubsection{Stellar birthrate}

Observations of the global star-formation rate in spiral galaxies suggest a Schmidt-law type of dependence of the stellar birthrate on some power of the total gas surface density $B \propto \Sigma_{\text {gas }}^{n}$ with $n \approx 1.5$ (Kennicutt 1998). An additional dependence of the star formation rate on the total surface density $\Sigma_{\text {tot }}(r, t)$ was suggested in self-regulating star formation theory (Talbot \& Arnett 1975). Later Dopita \& Ryder (1994) confirmed this by observations and suggested an empirical law of star formation $B(r, t) \propto \Sigma_{\mathrm{tot}}^{n} \Sigma_{\mathrm{g}}^{m}$ with $m=5 / 3$ and $n=1 / 3$ giving the best fit for the observed relationship between the stellar brightness and the surface brightness in $\mathrm{H}_{\alpha}$ in galactic disks. We adopt these values for the powers in the star formation law and like Alibes et al. (2001) choose the following form of the star formation rate:

$B(r, t)=v \frac{\sum_{\mathrm{tot}}(r, t)^{n} \Sigma_{\mathrm{g}}(r, t)^{m}}{\Sigma_{\mathrm{tot}}\left(r_{\odot}, t\right)^{n+m-1}}$ 
We take a star formation threshold into account, i.e. a minimum surface density $\Sigma_{\mathrm{g}}$ required for star formation, which is set to $7 M_{\odot} \mathrm{pc}^{-2}$ (Kennicutt 1998). In the numerical calculation, the transition from zero to the threshold value is smoothed to avoid the unphysical numerical oscillations in the solution for the surface density $\Sigma_{\mathrm{g}}$ close to the threshold that are otherwise produced by some integration methods. The constant $v$ is fitted such that the model fits the present-day star formation rate in the solar neighbourhood. We take $v=1.3 \mathrm{Gyr}^{-1}$.

\subsubsection{Stellar lifetimes}

We refuse the instantaneous recycling approximation, i.e. the assumption that massive stars die immediately after their birth and return metals to the ISM, and consider stellar lifetimes as a function of stellar mass and metallicity using an analytical approximation given by Reiteri et al. (1996). The formula of Reiteri et al. (1996) is a good fit for the stellar lifetimes computed by the Padova group (Alongi et al. 1993; Bressan et al. 1993; Bertelli et al. 1994) in the metallicity range $7 \times 10^{-5}<Z<3 \times 10^{-2}$ and for initial masses between 0.6 and $120 M_{\odot}$.

\subsubsection{Initial mass function}

The initial mass function (IMF) $\Phi(M)$ gives the distribution of stellar masses born in a star formation event. We adopt IMF consisting of four separate power-law type distributions in four separate intervals of initial masses proposed by Kroupa (2002):

$$
\Phi(M)=A\left\{\begin{array}{l}
C_{1} M^{-0.3}, 0.01 \leq M / M_{\odot}<0.08 \\
C_{2} M^{-1.3}, 0.08 \leq M / M_{\odot}<0.5 \\
C_{3} M^{-2.3}, 0.5 \leq M / M_{\odot}<1.0 \\
C_{4} M^{-2.7}, 1.0 \leq M / M_{\odot}<100,
\end{array}\right.
$$

where the coefficients $C_{1}=2.0158, C_{2}=0.1612$, and $C_{3}=C_{4}=$ 0.0806 are derived from the normalisation procedure. During the calculation we found that one obtains better model fits, if for high-mass stars, the exponent is changed to 2.55. This somewhat flatter power law $\left(\Phi \propto M^{-2.6}\right)$ is, for instance, observed for massive stars in the Orion nebula (Preibisch et al. 2002).

The average stellar mass is then given by the integration over the full range of stellar masses:

$$
M_{\mathrm{av}}=\int_{0.01}^{100} M \Phi(M) \mathrm{d} M .
$$

\subsubsection{Nucleosynthesis prescriptions}

The single low and intermediate mass stars from the mass range 0.8-8 $M_{\odot}$ contribute to the enrichment of the Milky Way with heavy elements due to excessive mass loss during the final stage of their AGB evolution. We adopt the yields for $\mathrm{H},{ }^{4} \mathrm{He},{ }^{12} \mathrm{C}$, ${ }^{13} \mathrm{C},{ }^{14} \mathrm{~N}$, and ${ }^{16} \mathrm{O}$ from van den Hoek \& Groenewegen (1997), tabulated for the range $0.8-8 M_{\odot}$ of initial masses and for metallicities from $10^{-3}$ to $4 \times 10^{-2}$. For ${ }^{23} \mathrm{Na},{ }^{24} \mathrm{Mg},{ }^{25} \mathrm{Mg},{ }^{26} \mathrm{Mg},{ }^{26} \mathrm{Al}$, and ${ }^{27} \mathrm{Al}$, the yields of Karakas et al. (2003) for the mass-range $1.0-6.5 M_{\odot}$ and range of metallicities $Z=0.004,0.008,0.02$ are used. The data are extrapolated outside the range of tables.

Rates of SN Ia explosions are calculated in the approximation of Matteucci \& Greggio (1986), based on the classical scenario of deflagration in C-O White Dwarfs in binary systems (Whelan \& Iben 1973), with a modification recently proposed by Hachisu et al. (1996, 1999) that accounts for the strong impact of metallicity on mass transfer in binaries to a compact object.

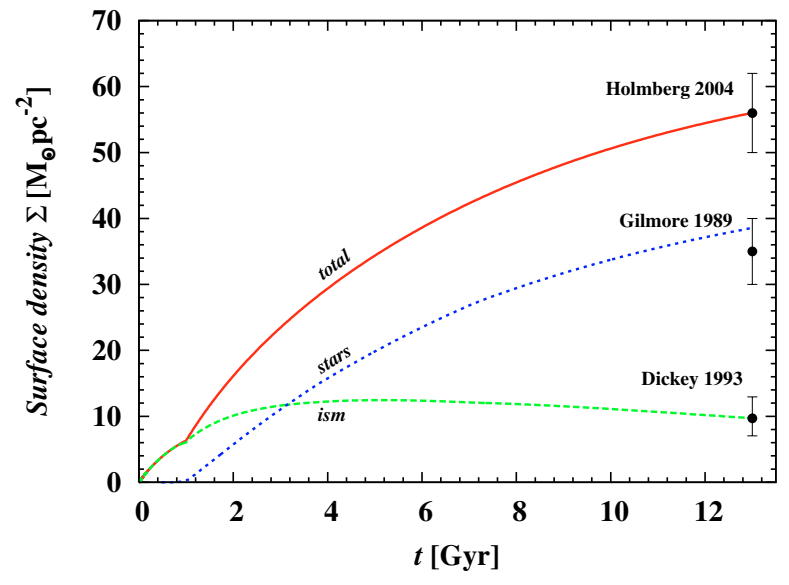

Fig. 1. Time evolution of the total surface density $\Sigma_{\text {tot }}$ (full line), of the surface density of visible stars (dotted line), and of the interstellar matter surface density $\Sigma_{\text {ISM }}$ (dashed line) of the galactic disk at the solar distance from the galactic centre, and observed values for the present day total surface density in the solar neighbourhood (Holmberg \& Flynn 2004), for the surface density of the stellar component (Gilmore et al. 1989), and for the surface density of the interstellar medium (Dickey 1993).

The SN Ia yields are taken from Iwamoto et al. (1999). The parameter determining the frequency of events is fitted such that the iron abundance of the Solar System is reproduced; a value of $\beta=2 \times 10^{-2}$ gives the best results.

The problem of yields from massive stars is complicated by the need to model supernova explosions, many details of which are still unknown. We adopt the recent nucleosynthesis prescriptions by Nomoto et al. (2006), which include two additional classes of SNe: very energetic Hypernovae and very faint and low-energy SNe. For comparison purposes, we also implemented the yields of Woosley \& Weaver (1995), which are most commonly used in chemical evolution calculations.

For massive stars with $M>40 M_{\odot}$, the mass returned by the stars up to the end of carbon burning is taken from the models of Schaller et al. (1993), Schaerer et al. (1993), and Charbonnel et al. (1993). It is assumed that the remaining mass collapses into a Black Hole. The mass return of nuclei is determined from the models for all those nuclei, whose surface abundances are given in the tables. For all others, we assume that their abundance in the returned mass equals their initial abundance.

It is assumed that the mass returned by stars is mixed with the general interstellar medium on much shorter timescales than the timescale for conversion into new stars; i.e., the composition of the interstellar medium is assumed to be homogeneous at each instant. This is justified by the observed low scatter of element abundances in the present ISM and of stellar element abundances in open stellar clusters (see Scalo \& Elmegreen 2004, and references therein).

\subsection{Evolution of some disk properties}

We now show some results of a numerical calculation of the galactic disk's chemical evolution at the solar circle that are important for our problem. For the model presented in the following the nucleosynthetic yields of massive stars are taken from the tables of Nomoto et al. (2006).

Figure 1 shows the evolution of the total surface mass density $\Sigma_{\text {tot }}\left(r_{\odot}, t\right)$ and that of the interstellar medium $\Sigma_{\text {ISM }}\left(r_{\odot}, t\right)$ for the galactic disk. In the model it is assumed that the formation 

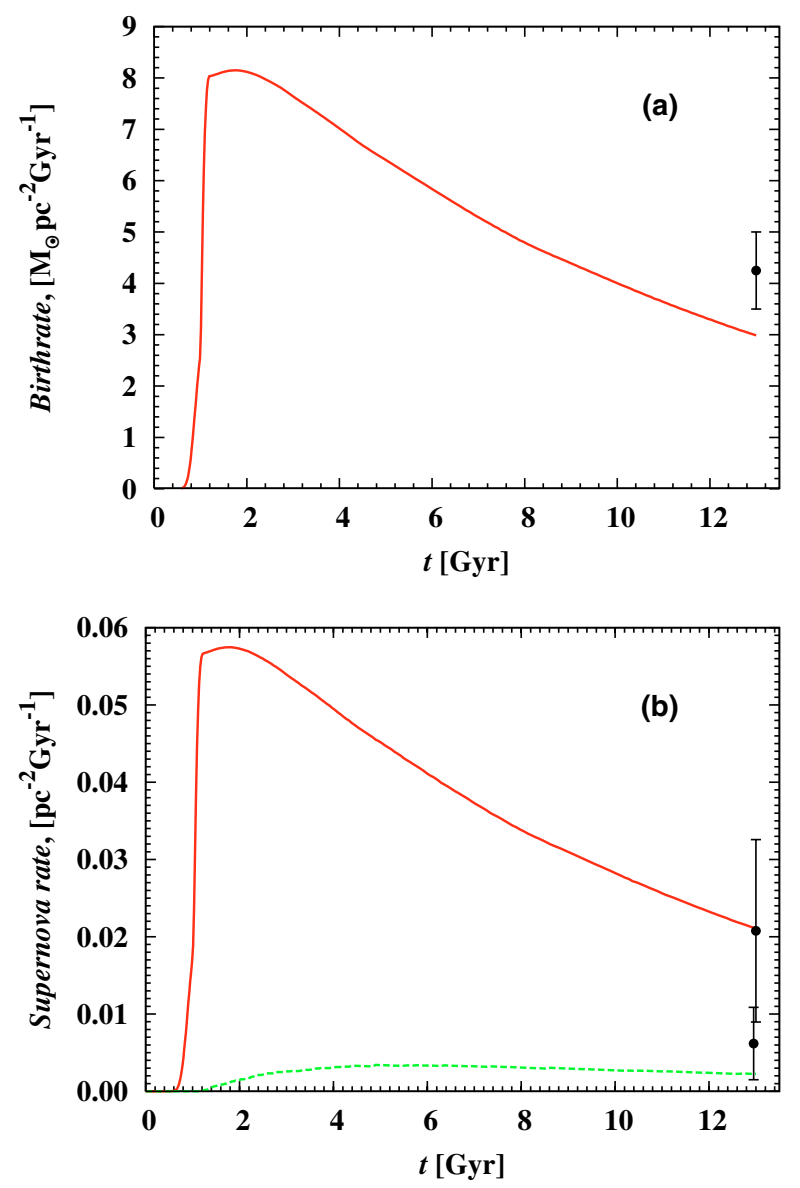

Fig. 2. a) Evolution of the astration rate $B$ at the solar galactocentric distance $r_{\odot}$. The error bar shows the presently observed stellar birthrate (Rana 1991). b) Evolution of the supernova type II (full line) and type Ia (dashed line) rates at the solar galactocentric distance $r_{\odot}$ and observed values at the present time from Tammann (1994).

of the disk started 13 Gyr ago. Initially most of the material in the disk was in gaseous interstellar matter, but today and at the time of Solar System formation most of the galactic matter is condensed into stars. A minor fraction is locked up in stellar remnants (White Dwarfs, Neutron Stars, Black Holes).

The evolution of the stellar birthrate is shown in Fig. 2a. Star formation commences about 1 Gyr after the onset of matter infall since about 1 Gyr time is required in the two-infall model until the gas density at the galactocentric distance of the sun increases to the threshold value for the star formation of $M_{\text {ISM }}=7 M_{\odot} \mathrm{pc}^{-2}$ (Kennicutt 1998). The stellar birthrate culminated about 2 Gyr after the onset of star formation, and since then it had gradually declined. Most of the stars born are low and intermediate mass stars. The massive stars mass fraction for the newly born stars is only $6.5 \%$ according to the initial mass function Eq. (5), but this small fraction is responsible for nearly all of the heavy nuclei synthesised in the Milky Way.

Figure $2 b$ shows the evolution of the supernova rates at the solar galactocentric distance $r_{\odot}$. Because of the short lifetime of massive stars, the supernova rate for type II supernovae closely resembles the birthrate of stars. Supernovae of type Ia appear with a delay of several Gyr because (i) their progenitors are longlived intermediate mass stars and (ii) supernova explosions in binaries are suppressed at low metallicities as proposed by Hachisu et al. $(1996,1999)$. Since supernovae of type Ia are the main

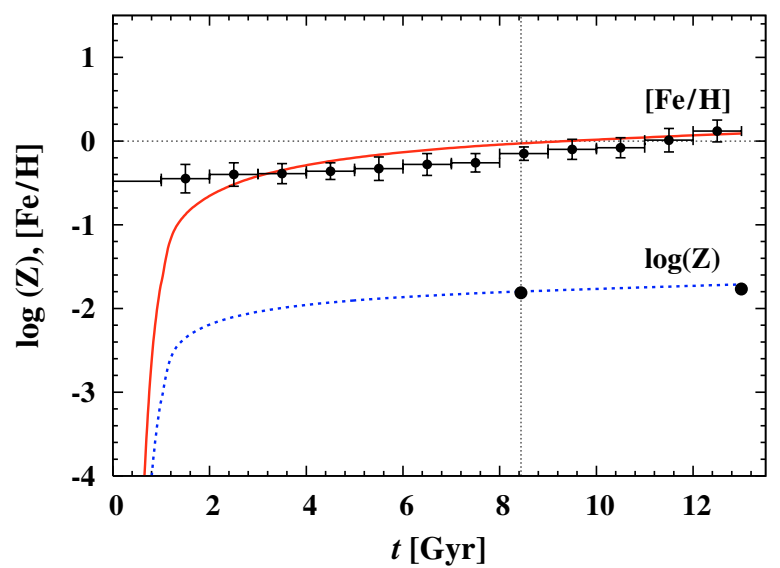

Fig. 3. Evolution of metallicity $Z$ of the ISM at the solar galactocentric distance $r_{\odot}$ and of the $[\mathrm{Fe} / \mathrm{H}]$ abundance ratio. The error bars show the observed age-metallicity relation from Rocha-Pinto (2000). The thin vertical line indicates the Solar System birth time which we assume to be $4.56 \mathrm{Gyr}$ ago, and the two filled circles indicate the observed metallicity of the sun and of the present-day ISM.

sources of Fe, the iron abundance increases in the Milky Way only on a rather long timescale.

\subsection{Chemical evolution of the disk}

The viability of galactic chemical-evolution models is usually tested by comparison with some standard observational constraints: G-dwarf metallicity distribution, age-metallicity relation, Solar System abundances at the instant of its formation $t_{\mathrm{SSF}}$, and evolution of element abundance ratios over time.

\subsubsection{Evolution of metallicity}

Figure 3 shows the time evolution of the metallicity $Z$ and the abundance ratio $[\mathrm{Fe} / \mathrm{H}]^{1}$ of the interstellar medium at the solar radius $r_{\odot}$. The predicted evolution of the $[\mathrm{Fe} / \mathrm{H}]$ ratio in the ISM is compared with the age-metallicity relation of the solar neighbourhood of late-type dwarfs from Rocha-Pinto (2000). The agemetallicity relation is reasonably well reproduced for the last about $10 \mathrm{Gyr}$, but there is an increasing discrepancy for earlier times. This is a general problem of all such evolution calculations and most likely stems from the unrealistically high stellar ages for many stars due to the rather crude methods of age determination.

All model calculations for the evolution of heavy element abundances with time predict a well-defined relation between metallicity and time-of-birth at a certain location in the galactic disk like the one shown in Fig. 3. Observationally determined ages obtained by comparing the position of a star in the Hertzsprung-Russel diagram with evolutionary isochrones, and relating spectroscopically determined metallicities of stars with such age determinations, show a tremendous scattering of metallicities for a given age. It has been concluded that this reflects (i) a true scattering of metallicities of the matter out of which stars

\footnotetext{
1 The abundance ratio $[\mathrm{X} / \mathrm{Y}]$ for two elements $\mathrm{X}$ and $\mathrm{Y}$ is defined as

$[\mathrm{X} / \mathrm{Y}]=\log \left(\frac{\epsilon_{\mathrm{X}}}{\epsilon_{\mathrm{Y}}}\right)-\log \left(\frac{\epsilon_{\mathrm{X}}}{\epsilon_{\mathrm{Y}}}\right)_{\odot}$

where $\epsilon_{\mathrm{X}}$ is the element abundance of element $\mathrm{X}$ by number relative to hydrogen.
} 


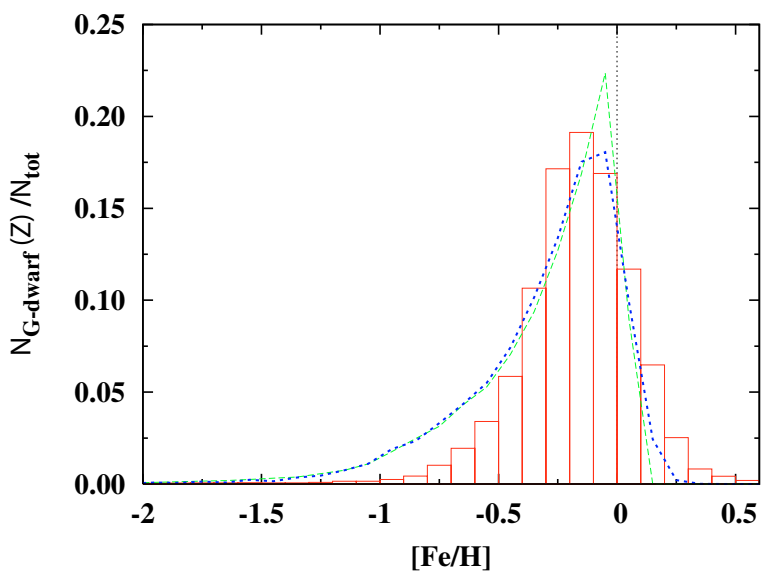

Fig. 4. G-dwarf metallicity distribution in the solar vicinity predicted by the model and the observed distribution as derived by Nordström (2004). The thin dashed line shows the G-dwarf distribution from direct calculations, while the thick dotted line is the result of a convolution with a Gaussian with half-width 0.2 dex to account for the observational scatter.

are formed at given galactocentric radius and birthtime, and (ii) possibly a mixing of stars from different galactic zones by radial diffusion (Edvardsson et al. 1993). Pont \& Eyer (2004) have shown, however, that the tremendous scattering most likely results from the difficulty of obtaining reliable stellar ages from evolutionary isochrones and that any true internal scattering of metallicities at given age is probably less than 0.15 dex. More careful analysis of age-metallicity relations based on such improved methods (da Silva et al. 2006) also seem to support a small intrinsic scattering of metallicities at any given age.

\subsubsection{Metallicity distribution of $\mathrm{G}$ dwarfs}

We compare the observed G-dwarf distribution from the most recent and most complete compilation of Nordström et al. (2004) with what is predicted by the model in Fig. 4. The dashed line shows the result of a convolution of the model results with a Gaussian with dispersion of 0.2 dex in order to simulate observational errors in the metallicity determination and intrinsic cosmic scatter in metal abundances. The errors of modern abundance determinations are usually claimed to be 0.1 dex or even less. The true scatter of stellar abundances for stars born at the same instant and location is difficult to determine, since neither the birthplace nor the birthtime of single stars is accurately known. The small scatter of abundances between stars in open stellar clusters indicate, however, that the intrinsic scatter seems to be very small (see Scalo \& Elmegreen 2004, and references therein); we arbitrarily assume a contribution of 0.1 dex to the total scatter.

The calculated metallicity distribution reproduces the general trends of the observed distribution, but it does not agree particular well. Significant deviations are seen for low and high metallicities. After convolution the discrepancies at the higher metallicity end disappear almost completely. For low metallicities the discrepancies persist and indicate that our model assumptions are not likely to be realistic for the earliest evolutionary phase. Since, for the main application of our model, this phase is not important, we did not try to improve the model in this respect.

\subsubsection{Evolution of abundances of individual elements}

Abundance ratios $[\mathrm{X} / \mathrm{Fe}]$ of elements $\mathrm{X}$ and their variation with time reflect the synthesis of heavy elements during galactic evolution. Reproducing these variations by the model is one of the most important tests of the model's reliability. For comparing the variation of $[\mathrm{X} / \mathrm{Fe}]$ with observed variations of stellar abundances, stellar ages would be required that are, however, unknown or of low accuracy. One prefers to compare instead the variation of the abundance ratios $[\mathrm{X} / \mathrm{Fe}]$ with the abundance ratio $[\mathrm{Fe} / \mathrm{H}]$, since $[\mathrm{Fe} / \mathrm{H}]$ is also determined from stellar atmosphere analysis and varies, at least for the Milky Way, monotonously with the age of the galactic disk (cf. Fig. 3), i.e., can be taken as a measure of stellar age. In our model we have calculated the evolution of 63 isotopes using nucleosynthesis prescription of Nomoto et al. (2006) and, for comparison, that of Woosley \& Weaver (1995). Results are presented in Fig. 5 for the elements related to dust formation. We concentrate here on these elements, since here we are mainly concerned with problems related to interstellar dust evolution.

The figure shows the model results if SN II yields from Nomoto et al. (2006) are used, and the corresponding results if yields from Woosley \& Weaver (1995) are used. The various dots, crosses etc. show results of stellar abundance analysis for $\mathrm{G}$ stars from the solar neighbourhood with the sources of data are given in the figure caption. These data show considerable scatter because of the errors in abundance determinations and possibly some small intrinsic scatter of element abundances of stars of comparable age. Nevertheless there are clear observable correlations between the abundance ratios $[\mathrm{X} / \mathrm{Fe}]$ and $[\mathrm{Fe} / \mathrm{H}]$. For the elements shown, the new results of Nomoto et al. give better agreement between the calculated abundance evolution and the observed correlations of $[\mathrm{X} / \mathrm{Fe}]$ with $[\mathrm{Fe} / \mathrm{H}]$ than the older Woosley \& Weaver results; for other elements, however, there are some discrepancies with observations.

There are some substantial problems with the yields of Woosley \& Weaver (1995). First, the iron yields of Woosley \& Weaver are too high, as already found in Timmes et al. (1995), and we follow their recommendation to reduce the Fe yield. Second, there is another severe problem with the Woosley \& Weaver results for magnesium. The calculated abundances based on the original yields are shown in Fig. 5 where these abundances are definitely too low, a familiar problem (e.g. Goswami \& Prantzos 2000; Francois et al. 2004). A comparison of the model results with the observed evolution of stellar magnesium abundances with metallicity shows that the shape of the $[\mathrm{Mg} / \mathrm{H}]-$ $[\mathrm{Fe} / \mathrm{H}]$-relation is reproduced reasonably well by the model, except that the absolute values of $[\mathrm{Mg} / \mathrm{H}]$ are systematically too low by a factor of 2.5. We therefore increased the $\mathrm{Mg}$ yields of Woosley \& Weaver (1995) by this factor in order to reproduce the $\mathrm{Mg}$ abundance of the Solar System. The resulting variation in $[\mathrm{Mg} / \mathrm{H}]$ with $[\mathrm{Fe} / \mathrm{H}]$ is shown in the figure and reproduces the observations much better. Such a correction would be necessary for calculating dust abundances, since reliable results for dust condensation require that the abundance ratios $\mathrm{Si} / \mathrm{Mg}$ and $\mathrm{Fe} / \mathrm{Si}$ of the main dust-forming elements agree with the observed abundance ratios in the Milky Way. Otherwise one would get a deviating dust mixture.

Since the yields of Nomoto et al. (2006) give results for the abundance evolution of the main dust-forming refractory elements much closer to observations than the Woosley \& Weaver (1995) yields, and since they do not require to introducing some ad hoc scaling, we prefer to use the Nomoto et al. (2006) yields for the model calculations. 


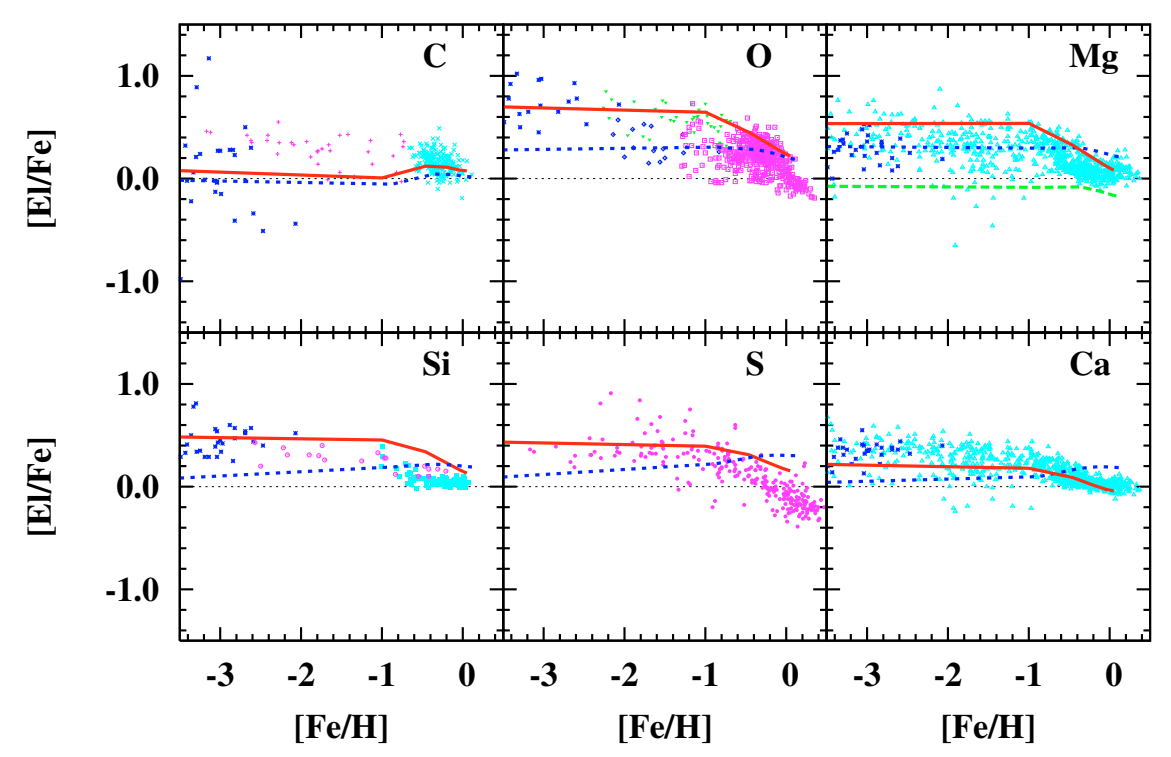

Fig. 5. Comparison of the predicted abundance ratios of the main dust-forming elements $[\mathrm{El} / \mathrm{Fe}]$ with observations of stellar abundances. The solid and dashed lines show model calculations with Nomoto (2006) and Woosley \& Weaver (1995) SNII yields, respectively. We corrected WW95 yields for Fe and $\mathrm{Mg}$ to achieve better fits to observations. For illustrative purposes, a model calculation with uncorrected Mg yields from WW95 is shown with a thin dashed line. The observed stellar element abundances for F and G stars from the solar neighbourhood are shown with different symbols for each of the sources (Akerman et al. 2004; Reddy et al. 2003; Soubiran et al. 2005; Melendez et al. 2002; Jonsell et al. 2005; Venn et al. 2004; Chen et al. 2000; Gratton et al. 1991; Caffau et al. 2005; Cayrel et al. 2004).

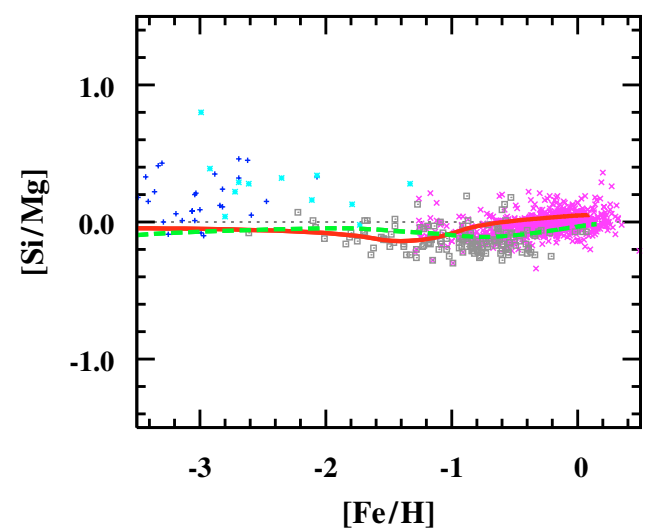

Fig. 6. Abundance ratio $\mathrm{Si} / \mathrm{Mg}$ of the major silicate dust forming elements. The full line corresponds to a model using SN yields of Nomoto et al. (2006), the dashed line to a model using SN yields from Woosley \& Weaver (1995). For the latter the $\mathrm{Mg}$ abundance is scaled such that it reproduces the solar $\mathrm{Mg}$ abundance at $[\mathrm{Fe} / \mathrm{H}]=0$.

Figure 6 compares the (adjusted) calculated abundance ratio $\mathrm{Si} / \mathrm{Mg}$ with observed abundance ratios in the atmospheres of nearby $\mathrm{F}$ and $\mathrm{G}$ stars and their correlation with metallicity $[\mathrm{Fe} / \mathrm{H}]$. The model results are close to the observed values. The $\mathrm{Si} / \mathrm{Mg}$ ratio determines the nature of the silicate dust that can be formed if $\mathrm{Mg}$ and $\mathrm{Si}$ are both completely condensed into dust. Oxygen is, in any case, abundant enough for to form of any kind of $\mathrm{Mg}-\mathrm{Si}$-compound. For a ratio of $\mathrm{Si} / \mathrm{Mg}=1$, one can form enstatite $\left(\mathrm{MgSiO}_{3}\right)$, for $\mathrm{Si} / \mathrm{Mg}=2$ one can form forsterite $\left(\mathrm{Mg}_{2} \mathrm{SiO}_{4}\right)$. For values in between, a mixture of both can be formed; here part of the $\mathrm{Mg}$ can be replaced by $\mathrm{Fe}$ and one instead forms a mixture of magnesium-iron-silicates. The stellar data show that the $\mathrm{Si} / \mathrm{Mg}$ ratio is close to unity for metallicities $[\mathrm{Fe} / \mathrm{H}]>-1$, and therefore one observes the formation of $\mathrm{Mg}-$ Fe-silicates in space.

\subsubsection{Solar System abundances}

The model should also reproduce the element abundances of the Solar System since they reflect the ISM composition at $r_{\odot}$ at the instant $t_{\mathrm{SSF}}=4.56 \mathrm{Gyr}$ ago, when the Sun was formed. Table 2 shows element abundances in the Solar System in the frequently used logarithmic scale ( $\epsilon$ is the abundance of an element relative to $\mathrm{H}$ by number)

$a=\log \epsilon+12$

for the elements from $\mathrm{H}$ to $\mathrm{Zn}$ that can be compared to the results of the model calculation if we use supernova yields from Woosley \& Weaver (1995) or Nomoto et al. (2006), since the tables only cover this range of elements. Element abundances for the Solar System are available from either spectroscopic abundance determinations from the solar photosphere or from laboratory analysis of primitive meteorites. Abundances for the solar photosphere given in the table are from the compilation of Asplund et al. (2005), except for He where the recommended value for the early sun from Grevesse \& Sauval (1998) is given. Abundances for meteorites are from the compilation of Palme \& Jones (2003). For C, N, and O abundances from Holweger (2001) are also given. For Ne and Ar see later. The last column indicates if solar photosphere abundances from Asplund et al. (S), or from Holweger (H), or meteoritic (M) abundances are preferred for comparison with model calculation results; a small letter indicates that the element is not used for a comparison in the present work.

The tabular values for the photosphere consider the recent significant downward revision of the abundances of $\mathrm{O}, \mathrm{C}$, and $\mathrm{N}$ by Allende Prieto et al. $(2001,2002)$ compared to the previous compilations of Grevesse \& Sauval (1998) and Anders \& Grevesse (1989). The table also gives the abundances for C, $\mathrm{N}$, and $\mathrm{O}$ derived by Holweger (2001), who also found a reduction in the solar abundances for these elements to be necessary, but not as reduced as in the papers by Allende Prieto et al. The abundances of Allende Prieto et al. pose serious problems 
Table 2. Solar system element abundances $a$ and the standard error of the abundance determination $\sigma$.

\begin{tabular}{|c|c|c|c|c|c|c|}
\hline \multirow[t]{2}{*}{$Z$} & \multirow[t]{2}{*}{ Elem. } & \multicolumn{2}{|c|}{ Sun } & \multicolumn{2}{|c|}{ Meteorites } & \multirow[t]{2}{*}{$\overline{\text { used }}$} \\
\hline & & $a$ & $\sigma$ & $a$ & $\sigma$ & \\
\hline 1 & $\mathrm{H}$ & 12.00 & - & & & $\mathrm{S}$ \\
\hline 2 & $\mathrm{He}$ & 10.99 & 0.02 & 1.92 & & $\mathrm{~S}$ \\
\hline 3 & $\mathrm{Li}$ & 1.05 & 0.10 & 3.30 & 0.04 & $\mathrm{~m}$ \\
\hline 4 & $\mathrm{Be}$ & 1.38 & 0.09 & 1.41 & 0.04 & $\mathrm{~m}$ \\
\hline 5 & B & 2.70 & 0.20 & 2.77 & 0.04 & $\mathrm{~m}$ \\
\hline \multirow[t]{2}{*}{6} & $\mathrm{C}$ & 8.39 & 0.05 & 7.39 & 0.04 & \\
\hline & & 8.59 & 0.11 & & & $\mathrm{H}$ \\
\hline \multirow[t]{2}{*}{7} & $\mathrm{~N}$ & 7.78 & 0.06 & 6.32 & 0.04 & \\
\hline & & 7.93 & 0.11 & & & $\mathrm{H}$ \\
\hline \multirow[t]{2}{*}{8} & $\mathrm{O}$ & 8.66 & 0.05 & 8.43 & 0.04 & \\
\hline & & 8.74 & & & & $\mathrm{H}$ \\
\hline 9 & $\mathrm{~F}$ & 4.56 & 0.30 & 4.45 & 0.06 & $\mathrm{~m}$ \\
\hline 10 & $\mathrm{Ne}$ & 8.08 & 0.07 & & & $\mathrm{~S}$ \\
\hline 11 & $\mathrm{Na}$ & 6.17 & 0.04 & 6.30 & 0.02 & M \\
\hline 12 & $\mathrm{Mg}$ & 7.53 & 0.09 & 7.56 & 0.01 & M \\
\hline 13 & $\mathrm{Al}$ & 6.37 & 0.06 & 6.46 & 0.01 & M \\
\hline 14 & $\mathrm{Si}$ & 7.51 & 0.04 & 7.55 & 0.01 & M \\
\hline 15 & $\mathrm{P}$ & 5.36 & 0.04 & 5.44 & 0.04 & $\mathrm{~S}$ \\
\hline 16 & $\mathrm{~S}$ & 7.14 & 0.05 & 7.19 & 0.04 & M \\
\hline 17 & $\mathrm{Cl}$ & 5.50 & 0.30 & 5.26 & 0.06 & $\mathrm{~s}$ \\
\hline 18 & $\mathrm{Ar}$ & 6.70 & 0.06 & & & $\mathrm{~s}$ \\
\hline 19 & $\mathrm{~K}$ & 5.08 & 0.07 & 5.11 & 0.02 & M \\
\hline 20 & $\mathrm{Ca}$ & 6.31 & 0.04 & 6.33 & 0.01 & M \\
\hline 22 & $\mathrm{Ti}$ & 4.90 & 0.06 & 4.95 & 0.04 & M \\
\hline 23 & V & 4.00 & 0.02 & 3.99 & 0.02 & M \\
\hline 24 & $\mathrm{Cr}$ & 5.64 & 0.10 & 5.67 & 0.01 & M \\
\hline 25 & $\mathrm{Mn}$ & 5.39 & 0.03 & 5.51 & 0.01 & M \\
\hline 26 & $\mathrm{Fe}$ & 7.45 & 0.05 & 7.49 & 0.01 & M \\
\hline 27 & Co & 4.92 & 0.08 & 4.90 & 0.01 & M \\
\hline 28 & $\mathrm{Ni}$ & 6.23 & 0.04 & 6.23 & 0.02 & M \\
\hline 29 & $\mathrm{Cu}$ & 4.21 & 0.04 & 4.28 & 0.04 & M \\
\hline 30 & $\mathrm{Zn}$ & 4.60 & 0.03 & 4.66 & 0.04 & M \\
\hline
\end{tabular}

for solar helioseismology (Delahaye \& Pinsonneault 2006; Basu et al. 2007) while the abundances of Grevesse \& Sauval (1998) give good fits to observations. The incompatibility of the new $\mathrm{C}, \mathrm{N}, \mathrm{O}$ abundances with helioseismological results should be taken seriously and the abundance reductions following from using numerically calculated models for the solar convective flows (Asplund et al. 2000) to determine spectral line profiles seem to result in unrealistically small abundances. Comparison with abundances in nearby $\mathrm{G}$ stars also seems to indicate this (see Sect. 2.3.5). The spatial resolution of their flow calculations of about $50 \mathrm{~km}$ (Asplund et al. 2000) compared to a pressure scale height of the solar photosphere of about $125 \mathrm{~km}$ probably is insufficient and does not account for velocity fluctuations on length scales that are small compared to the mean free path-lengths of photons and therefore produces incorrect equivalent widths.

No reliable abundances of the noble gases can be determined for the solar photosphere. For He a photospheric abundance is given in the table, which is the value recommended by Grevesse \& Sauval (1998) to be taken as the value of the He abundance of the early sun; the He abundance of the present sun is lower due to segregation effects and cannot be used for a comparison. The abundances for the other noble gases given in the table are determined from the $\mathrm{Ne} / \mathrm{Mg}$ and $\mathrm{Ar} / \mathrm{Mg}$ abundance ratios determined from coronal lines as given by Feldman \& Widing (2003). It is not sure that they really correspond to the initial solar abundances.

For meteorites the abundance of $\mathrm{H}$ and of the noble gases do not reflect their abundance in the material out of which the

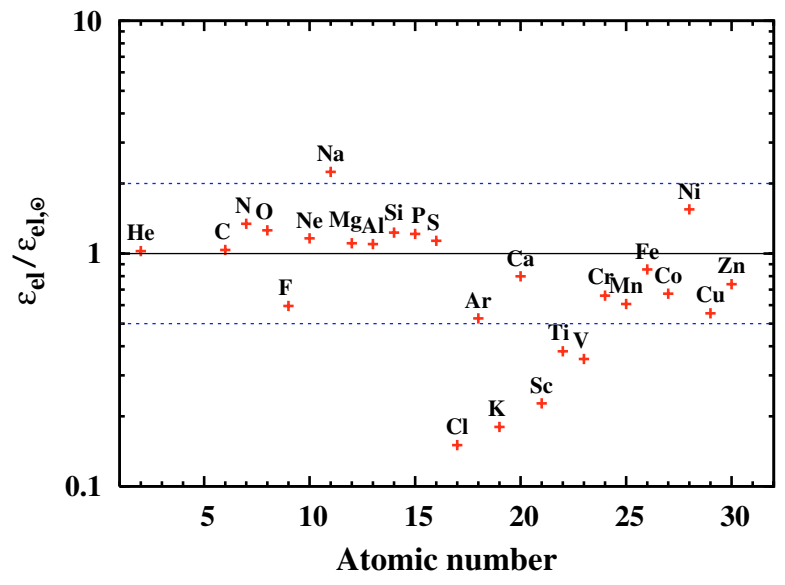

Fig. 7. Calculated element abundances relative to the solar abundances at the instant of Solar System formation (data according to Table 2). Thin dotted lines show the as much as twice a deviation from observed values.

parent bodies of the meteorites formed since these elements are not incorporated into the bodies of the early Solar System. Therefore no data for meteoritic abundances are given in the table for these elements.

For meteorites the abundances of the volatile elements $\mathrm{C}, \mathrm{N}$, and $\mathrm{O}$ are also not representative for the abundances in the early Solar System since these elements are not $(\mathrm{N})$ or only to a small fraction $(\mathrm{C}, \mathrm{O})$ condensed into solids and incorporated into the parent bodies of the meteorites. Correspondingly, the meteoritic abundances of $\mathrm{C}, \mathrm{N}$, and $\mathrm{O}$ given in the table are much lower than the photospheric abundances. For these elements the solar photospheric abundances have to be used for comparison purposes. A number of elements are highly volatile (cf., e.g., Palme \& Jones 2003) and it is doubtful that these elements are completely condensed in the parent bodies of the meteorites. Besides $\mathrm{H}$, the noble gases, and $\mathrm{C}, \mathrm{N}$, and $\mathrm{O}$, these are the elements $\mathrm{Cl}, \mathrm{Br}, \mathrm{I}$, $\mathrm{In}, \mathrm{Cs}, \mathrm{Hg}, \mathrm{Tl}, \mathrm{Pb}, \mathrm{Bi}$, from which $\mathrm{Cl}$ is one of the elements in the table. For comparison purposes one should therefore use the $\mathrm{Cl}$ abundance from the photosphere, but since the abundance determination of $\mathrm{Cl}$ for the solar photosphere is rather inaccurate, $\mathrm{Cl}$ is presently not suited for comparison.

For the remaining elements, the meteoritic and the solar photospheric abundances agree closely, except for a number of heavier elements not contained in our table. For comparison with the results of the chemical evolution calculation, we usually preferred the more accurate meteoritic abundance, whereas the solar photospheric was used when both methods were of only moderately accurate (as specified in last column of Table 2). Additionally, the elements $\mathrm{Li}, \mathrm{Be}, \mathrm{B}, \mathrm{F}$ are excluded from the comparison, since their production mechanisms are not implemented in the model program.

In Fig. 7 we present the predicted element abundances of the ISM relative to Solar System abundances at the instant of Solar System formation at $r=r_{\odot}$. Thin horizontal lines indicate a deviation by a factor of two upward or downward from Solar System abundances. As can be seen, the model fits the observed abundances with good accuracy. Most calculated element abundances reproduce the Solar System element abundances within a factor of about two, many elements even much better. The somewhat worse results for $\mathrm{Cl}, \mathrm{K}$, and $\mathrm{Sc}$ have also be found by Kobayashi et al. (2006); the rather bad results for these elements are of no importance for our model, since they are not one of the main dust-forming elements. 
Table 3. Average abundances $a$ of $\mathrm{F}$ and $\mathrm{G}$ stars with solar metallicity $(|\Delta[\mathrm{Fe} / \mathrm{H}]|<0.05)$ and of young stars (age $\leq 1 \mathrm{Gyr}$ ) from the solar neighbourhood. $\sigma_{\mathrm{abd}}$ is the accuracy of the abundance determinations from stellar spectra, $\sigma_{*}$ is the scattering of the stellar abundances around the mean. $Z$ is the metallicity calculated from these abundances.

\begin{tabular}{|c|c|c|c|c|c|c|c|c|c|c|}
\hline \multirow[b]{3}{*}{$\mathrm{Z}$} & \multirow[b]{3}{*}{ Element } & \multicolumn{6}{|c|}{ Nearby F \& G stars } & \multicolumn{3}{|c|}{ B dwarfs } \\
\hline & & \multicolumn{3}{|c|}{ solar met. } & \multicolumn{2}{|c|}{ age $<1 \mathrm{Gyr}$} & \multirow[b]{2}{*}{ Source } & & & \\
\hline & & $a$ & $\sigma_{\text {abd }}$ & $\sigma_{*}$ & $a$ & $\sigma_{*}$ & & $a$ & $\sigma_{*}$ & Source \\
\hline 2 & $\mathrm{He}$ & & & & & & & 11.02 & 0.05 & 3 \\
\hline 6 & $\mathrm{C}$ & 8.37 & 0.06 & 0.11 & 8.39 & 0.11 & 2 & 8.32 & 0.10 & 4 \\
\hline 7 & $\mathrm{~N}$ & & & & & & & 7.73 & 0.28 & 5 \\
\hline 8 & $\mathrm{O}$ & 8.75 & & 0.07 & 8.77 & 0.13 & 1 & 8.63 & 0.18 & 4 \\
\hline 10 & $\mathrm{Ne}$ & & & & & & & 8.11 & 0.04 & 6 \\
\hline 11 & $\mathrm{Na}$ & 6.30 & 0.03 & 0.16 & 6.27 & 0.10 & 1 & & & \\
\hline 12 & $\mathrm{Mg}$ & 7.63 & 0.06 & 0.32 & 7.64 & 0.21 & 1 & 7.59 & 0.15 & 7 \\
\hline 13 & $\mathrm{Al}$ & 6.52 & 0.05 & 0.24 & 6.54 & 0.22 & 1 & 6.24 & 0.14 & 5 \\
\hline 14 & $\mathrm{Si}$ & 7.60 & 0.05 & 0.28 & 7.61 & 0.23 & 1 & 7.50 & 0.21 & 5 \\
\hline 16 & $\mathrm{~S}$ & 7.17 & 0.16 & & 7.29 & 0.10 & & 7.22 & 0.10 & 4 \\
\hline 18 & $\mathrm{Ar}$ & & & & & & & 6.48 & 0.04 & 8 \\
\hline 20 & $\mathrm{Ca}$ & 6.42 & 0.03 & 0.37 & 6.48 & 0.39 & 1 & & & \\
\hline 22 & $\mathrm{Ti}$ & 4.92 & 0.03 & 0.11 & 4.94 & 0.14 & 1 & & & \\
\hline 24 & $\mathrm{Cr}$ & 5.66 & 0.02 & 0.13 & 5.73 & 0.28 & 1 & & & \\
\hline 26 & $\mathrm{Fe}$ & 7.55 & 0.06 & 0.12 & 7.61 & 0.25 & 1 & 7.46 & 0.08 & 9 \\
\hline 28 & $\mathrm{Ni}$ & 6.22 & 0.02 & 0.09 & 6.25 & 0.07 & 1 & & & \\
\hline 30 & $\mathrm{Zn}$ & 4.53 & 0.06 & 0.27 & 4.54 & 0.13 & 1 & & & \\
\hline & $Z$ & 0.0194 & & & 0.0203 & & & 0.168 & & \\
\hline
\end{tabular}

Sources: (1) Bensby et al. (2005), (2) Bensby \& Feltzing (2006), (3) Lyubimkov et al. (2004), (4) Daflon \& Cunha (2004), (5) Rolleston et al. (2000), (6) Cunha et al. (2006), (7) Lyubimkov et al. (2005), (8) Holmgren et al. (1990), (9) Cunha \& Lambert (1994).

\subsubsection{Abundances of $F \& G$ stars}

Abundances of the ISM cannot be measured directly because in the ISM the refractory elements are condensed into dust (cf. Savage \& Sembach 1996). One possibility to indirectly determine total element abundances in the ISM is to determine atmospheric abundances from some kind of "young" stars that have not changed their surface abundances since they formed from interstellar matter. Best-suited for this purpose are probably $\mathrm{F}$ and $\mathrm{G}$ main sequence stars from the galactic neighbourhood, that show the kinematics of thin disk stars and high metallicities, or, if stellar ages have been determined, have an age of no more than a few Gyr. For such stars one can assume that they formed from interstellar material with essentially the same properties as the present-day ISM of the galactic neighbourhood. Bensby et al. (2005) and Bensby \& Feltzing (2006) determined recently abundances for a number of elements for thin and thick disk stars from the solar neighbourhood. From the elements considered in that paper, the following are relevant for our purposes: $\mathrm{C}, \mathrm{O}, \mathrm{Na}$, $\mathrm{Mg}, \mathrm{Al}, \mathrm{Si}, \mathrm{Ca}, \mathrm{Ti}, \mathrm{Cr}, \mathrm{Fe}, \mathrm{Ni}$, and $\mathrm{Zn}$.

First, we consider from this sample the stars with an $[\mathrm{Fe} / \mathrm{H}]$ ratio within \pm 0.05 of the solar value. There are 6 stars that satisfy this condition, and Table 3 shows the average abundances $a$ of the above elements and the average scattering $\sigma_{*}$ of the abundances around the mean value. For comparison the table also shows the random errors of the abundance determinations from stellar spectra as given by the authors. These abundances are surprisingly close to the Solar System abundances, though the general metallicity is somewhat higher. If the range of metallicities is increased to \pm 0.1 of the solar $[\mathrm{Fe} / \mathrm{H}]$ ratio, the number of stars increases to 13 , but the average values for the abundances are practically unchanged; i.e., the average abundances given for solar like stars in the table do not depend substantially on the precise choice of the limit $\Delta[\mathrm{Fe} / \mathrm{H}]$. Hence abundances of F \& G stars with Solar System metallicity agree rather well with Solar System abundances as given in Table 2, which have already been compared in Fig. 7 with results of our model

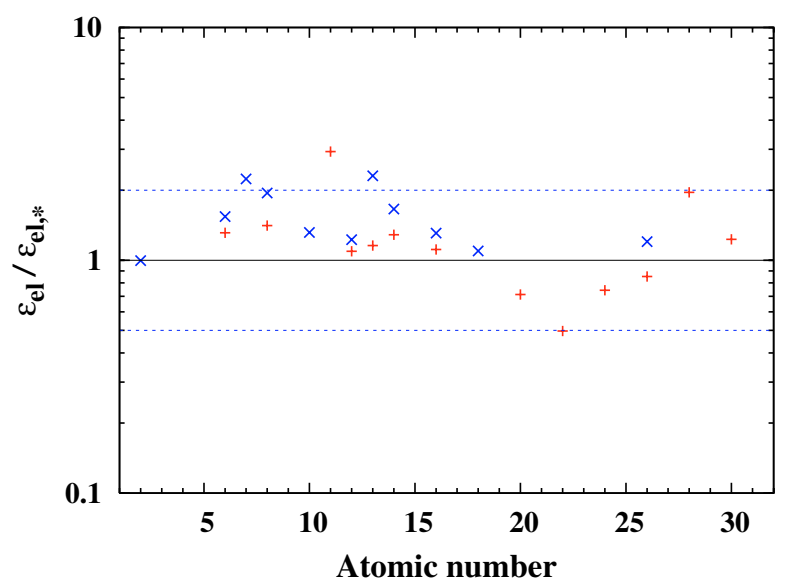

Fig. 8. Calculated element abundances relative to the abundances of $F$ \& $\mathrm{G}$ stars from the solar vicinity, with ages less than $1 \mathrm{Gyr}$ (pluses), and of B stars from the range $r_{\odot} \pm 2 \mathrm{kpc}$ of the galactocentric distances (crosses) (data according to Table 3). Thin dotted lines show the as much as twice a deviation from observed values.

calculation. Our model therefore reproduces the observed abundances of solar metallicity stars reasonably well at the solar circle.

Second we choose from the sample of Bensby et al. (2005) and Bensby \& Feltzing (2006) the thin disk stars with ages less than 1 Gyr. Despite the large uncertainties of such age determinations, it seems likely that these stars belong to the most recently born stars in the sample of thin disk stars. Their abundances should therefore sample the abundance of the ISM in the solar vicinity during the last, e.g., $1 \ldots 2 \mathrm{Gyr}$ or so. The average abundances of the elements determined by Bensby et al. (2005) and Bensby \& Feltzing (2006) for these stars are given in Table 3. Extending the sample to stars with ages less than $2 \mathrm{Gyr}$ does not result in significant changes in the average abundances; i.e., the results do not depend on the precise choice of the age 


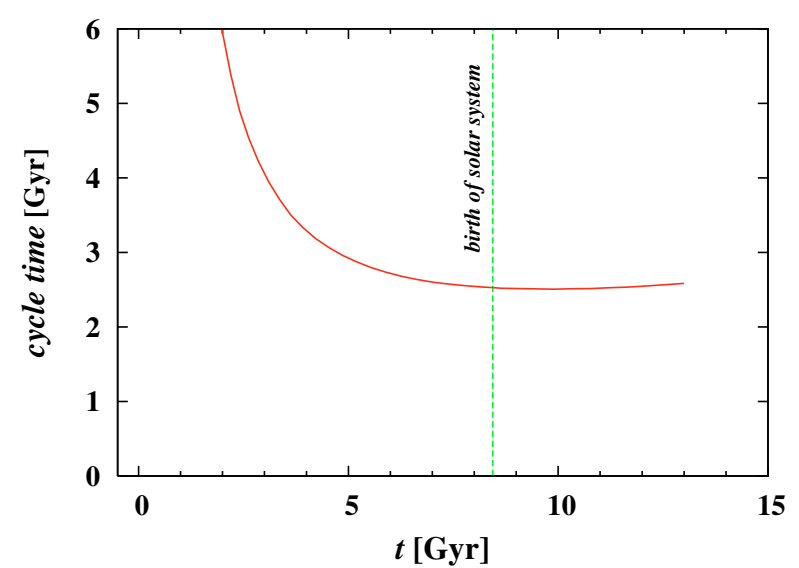

Fig. 9. Characteristic astration timescale for conversion of interstellar matter into stars at the solar circle.

limit. The typical metallicity $Z$ of the "present" ISM determined in this way (the contribution of $\mathrm{N}$ and $\mathrm{Ne}$ to $Z$ is estimated) is slightly higher than the Solar System metallicity, as one may expect from ongoing element synthesis. Our model results for the present day ISM abundances are compared in Fig. 8 with the observed abundances of F \& G stars formed within the last Gyr given in Table 3. Our model results for the present ISM are also in good agreement with observations.

\subsubsection{Abundances of $B$ dwarfs}

Stars of early spectral type B have short lifetimes, so they sample abundances from the present-day thin disk. Abundances have been determined in particular for B stars in stellar clusters and we show in Table 3 average abundances taken from the literature for B dwarfs in stellar cluster with galactocentric distances from a range of $\pm 2 \mathrm{kpc}$ around the solar circle. Despite the rather heterogeneous observational material the scattering of observed abundances around the mean is moderate; i.e., element abundances in the ring $8 \pm 2 \mathrm{kpc}$ around the galactic centre seem to be quite homogeneous. The average abundances and, thus, the metallicity $Z$, are typically slightly less than the presentday abundances found from F and G stars (see Fig. 8), as also found by Sofia \& Meyer (2001). Figure 8 compares our calculated abundances for the current ISM with the abundances of a $\mathrm{B}$ dwarf; the agreement again is reasonable, but compared to the case of F \& G stars it is worse since abundances of B dwarfs are less than for $F \& G$ stars.

\subsection{Astration time for the interstellar matter}

In the context of dust evolution, an important quantity is the timescale for conversion of interstellar matter into stars, the astration timescale. This is given by

$\tau_{\text {cycle }}=\frac{\Sigma_{\mathrm{ISM}}}{B}$.

This quantity is shown in Fig. 9. At the instant of forming of the Solar System, the astration timescale was about 2.5 Gyr.

\section{Production rates for stardust}

In the following we study the evolution of the interstellar dust in the Milky Way disk within the frame of the simple one-zone approximation for the galactic disk evolution described above. The
Table 4. Dust species considered in the model calculation.

\begin{tabular}{lcccc}
\hline \hline & Silicates & Carbon & SiC & Iron \\
\hline AGB stars & $\sqrt{ }$ & $\sqrt{ }$ & $\sqrt{ }$ & $\sqrt{ }$ \\
SN II & $\sqrt{ }$ & $\sqrt{ }$ & $\sqrt{ }$ & $\sqrt{ }$ \\
SN Ia & - & - & - & $\sqrt{ }$ \\
ISM & $\sqrt{ }$ & $\sqrt{ }$ & - & $\sqrt{ }$ \\
\hline
\end{tabular}

model follows the principles of the Dwek (1998) model, which was the first one that coupled dust evolution consistently with a full model for the chemical evolution of the Milky Way. The present model calculation concentrates on the most abundant dust components formed in stars that are also found as presolar dust grains in meteorites:

$$
\begin{aligned}
& \text { - silicate dust; } \\
& \text { - carbon dust; } \\
& \text { - silicon carbide. }
\end{aligned}
$$

It also considers

- solid iron

though that not yet has been identified as a presolar dust species. For theoretical reasons it should, however, be an abundant dust species produced in stellar outflows.

\subsection{Evolution model for the interstellar dust}

In our evolution model we consider a number of different dust species, denoted by an index $j$.

First, we differentiate between dust coming from different types of parent stars. Even if the chemical composition of a certain dust species formed in outflows or ejecta of different stellar types is the same, the individual grains of this dust species are carriers of the isotopic anomalies corresponding to the particular nuclear processes operating in their parent stars. If they are investigated in the laboratory as presolar dust grains, one can, at least in principle, identify the formation site for every grain. This makes it desirable to count dust species from different types of stars with the same overall chemical composition but with different types of isotopic anomalies as different species $j$. Presently we choose not too fine a division into stellar types and consider three different kinds of stellar sources: supernovae of type II, supernovae of type Ia, and AGB stars. Supernovae of type II and AGB stars can form all of the four chemically different types of dust considered in our model, while supernovae of type Ia can probably form only iron dust (if ever). Hence we consider in our model nine different kinds of stardust coming from three different types of parent stars.

Second, we consider dust formed in the interstellar medium itself. From the element abundances in the interstellar medium, one expects that silicate dust can be formed. Observations of the interstellar dust indicate that carbon dust can also be formed in certain regions of the interstellar medium. It seems unlikely, however, that $\mathrm{SiC}$ dust can be formed, since this requires a carbon-rich environment, which is not encountered in interstellar space. Iron dust may also be formed in the ISM, though this element is probably consumed mainly by silicate formation. Hence we consider in our model three kinds of dust formed in the interstellar medium: silicate, iron, and carbon dust. In all, our model considers the twelve different kinds of dust from stellar sources and the interstellar medium given in Table 4. 
We describe the abundance of each dust component $j$ in the interstellar medium by its surface mass density $\Sigma_{j, \mathrm{~d}}$. The evolution of the surface density is determined by the equation

$\frac{\mathrm{d} \Sigma_{j, \mathrm{~d}}}{\mathrm{~d} t}=-\frac{\Sigma_{j, \mathrm{~d}}}{\Sigma_{\mathrm{ISM}}} B+\sum_{l} R_{j, l, \mathrm{~d}}-L_{j, \mathrm{~d}}+G_{j, \mathrm{~d}}$.

The first term on the r.h.s. describes the loss of dust from the interstellar medium by star formation. It is assumed that only the dust content of the matter that is converted into stars is destroyed and that no additional dust is destroyed during this process. Also no return of freshly-formed dust from protostellar disks by winds or jets is assumed to occur, though this has been speculated possibly to be important (Tielens 2003). The second term on the r.h.s. describes the amount of dust of kind $j$ injected by stars of type $l$ into the interstellar medium. The third term on the r.h.s. describes the losses, the destruction of dust of kind $j$ in the interstellar medium, mainly by supernova shocks, and the last term describes the gain, the formation of dust of kind $j$ in the interstellar medium by growth processes in molecular clouds. We assume no contribution of dust from the infall in our model.

From Eqs. (9), one calculates the surface mass density of the different dust species $j$. Additionally, one has the set of equations for the total surface densities $\Sigma_{i}$ of each element $i$ in the ISM. The surface density $\Sigma_{i, \mathrm{~g}}$ of each element in the gas phase of the ISM then follows as the difference between its total surface density $\Sigma_{i}$ and the sum of the contributions of all dust species containing that element,

$\Sigma_{i, \mathrm{~g}}=\Sigma_{i}-\sum_{j} v_{i j} \frac{A_{i}}{A_{j, \mathrm{~d}}} \Sigma_{j, \mathrm{~d}}$.

Here $v_{i j}$ is the number of atoms of element $i$ in one formula unit ${ }^{2}$ of dust species $j, A_{i}$ and $A_{j, \mathrm{~d}}$ are the atomic weights of element $i$ and of one formula unit of dust species $j$, respectively, and the summation is over all dust species containing element $i$.

In the following we describe the details of the processes relevant for the evolution of interstellar dust abundances and how they are implemented in the model.

\subsection{Dust return by $A G B$ stars}

The main factories of dust in space are low and intermediate mass stars in the AGB stage of their evolution. These are stars with initial masses between about $0.8 M_{\odot}$ and about $8 M_{\odot}$, which end their life as White Dwarfs. The lower mass limit corresponds to that initial mass, for which the lifetime of a star corresponds to the age of the Milky Way. The upper mass limit corresponds to stars that finally explode as supernovae and do not evolve through an AGB phase ${ }^{3}$.

The initial element mixture of all stars is oxygen rich in the sense that the abundance $\epsilon_{\mathrm{O}}$ of oxygen exceeds the abundance $\epsilon_{C}$ of carbon. This does not change during their whole evolution up to the thermally pulsing AGB (TP-AGB), despite some abundance changes during the first and second dredge-ups on the Red Giant Branch and the early AGB, respectively. If the third dredge-up process starts operating, the ashes of He burning are mixed to the convective envelope of the star after each

\footnotetext{
2 The formula unit is the fictitious molecular group in the solid corresponding to the chemical formula of the condensed phase.

3 Presently we neglect the possibility that stars from the region of initial masses $8<M<12 M_{\odot}$ may become super-AGB stars (e.g. Gil-Pons \& García-Berro 2002)
}

thermal pulse, increasing the carbon abundance of the convective envelope stepwise, but only marginally changes its oxygen abundance. The resulting evolution of the carbon-to-oxygen ratio $\epsilon_{\mathrm{C}} / \epsilon_{\mathrm{O}}$ on the TP-AGB depends on the initial mass of the stars:

(1) Low mass stars with initial masses less than about $M=$ $1.5 M_{\odot}$ lose their envelope by a stellar wind before the carbon abundance exceeds the oxygen abundance. Only for low metallicities does their envelope become carbon rich prior to complete envelope loss. For very low initial masses, probably no third dredge-up occurs and the stars never become carbon stars.

(2) Stars from the range of initial masses between about $M=$ $1.5 M_{\odot}$ and about $M=4 M_{\odot}$ increase their carbon abundance over the oxygen abundance after a number of thermal pulses and become carbon rich. They evolve further as carbon stars until their envelope is lost by the stellar wind.

(3) Intermediate mass stars with initial masses between about $M=4 M_{\odot}$ and about $M=8 M_{\odot}$ convert the dredged-up carbon rapidly into ${ }^{14} \mathrm{~N}$ via the $\mathrm{CN}$-cycle, since the lower part of their convective envelope overlaps with the upper edge of the H-burning shell. The oxygen, however, is not affected by this process, and for this, the carbon abundance in the envelope of these stars is much less than the oxygen abundance. They do not become carbon stars until most of their envelope is lost, and the convection zone of the remaining envelope no longer overlaps with the H-burning shell. For a short period the stars then also become carbon stars until finally the last portion of their envelope is removed by the stellar wind.

Depending on the $\mathrm{C} / \mathrm{O}$ abundance ratio, the stars produce different dust mixtures in their outflows. Here we are only interested in the dominating dust species, which are formed from the most abundant elements, and so concentrate on the following four types of dust: silicates, carbon, silicon carbide, and iron. The dust production rate over the whole period of AGB evolution for these dust species was calculated by Ferrarotti \& Gail (2006).

The silicate dust is only produced during the oxygen-rich phase of the stellar evolution where the stars spectroscopically appear as M stars. Some minor fractions are also produced during the $\mathrm{S}$ star phase where the $\mathrm{C} / \mathrm{O}$ abundance ratio is close to unity. The silicates are a mixture of olivine- and pyroxenetype amorphous dust and, for part of the stars, also up to about $15 \%$ of nearly iron free crystalline forsterite and enstatite is observed to be formed (cf. the review of Molster \& Waters 2003). The present work does not distinguish between the two types of amorphous silicate dust since for silicate dust in the interstellar medium it is presently not possible to distinguish by observations of the dust absorption spectrum unambiguously between the two different components (cf. the contradictory results in Chiar \& Tielens 2006; Min et al. 2007). Also, crystalline Mgsilicates are not considered since they are not found in the interstellar medium (Kemper et al. 2004), possibly because they are rapidly amorphized in the ISM after their ejection by interaction with energetic electrons and ions (cf. Demyk et al. 2004; Jäger et al. 2003).

Carbon and silicon carbide dust are produced by AGB stars during their carbon rich phase of evolution on the AGB where they spectroscopically appear as C stars. Iron dust is included in the model calculation, though it has not yet been unambiguously identified as a major dust component in stellar outflows; only some hints of its existence have been found up to now (e.g. Kemper et al. 2002). This is because no readily identifiable 

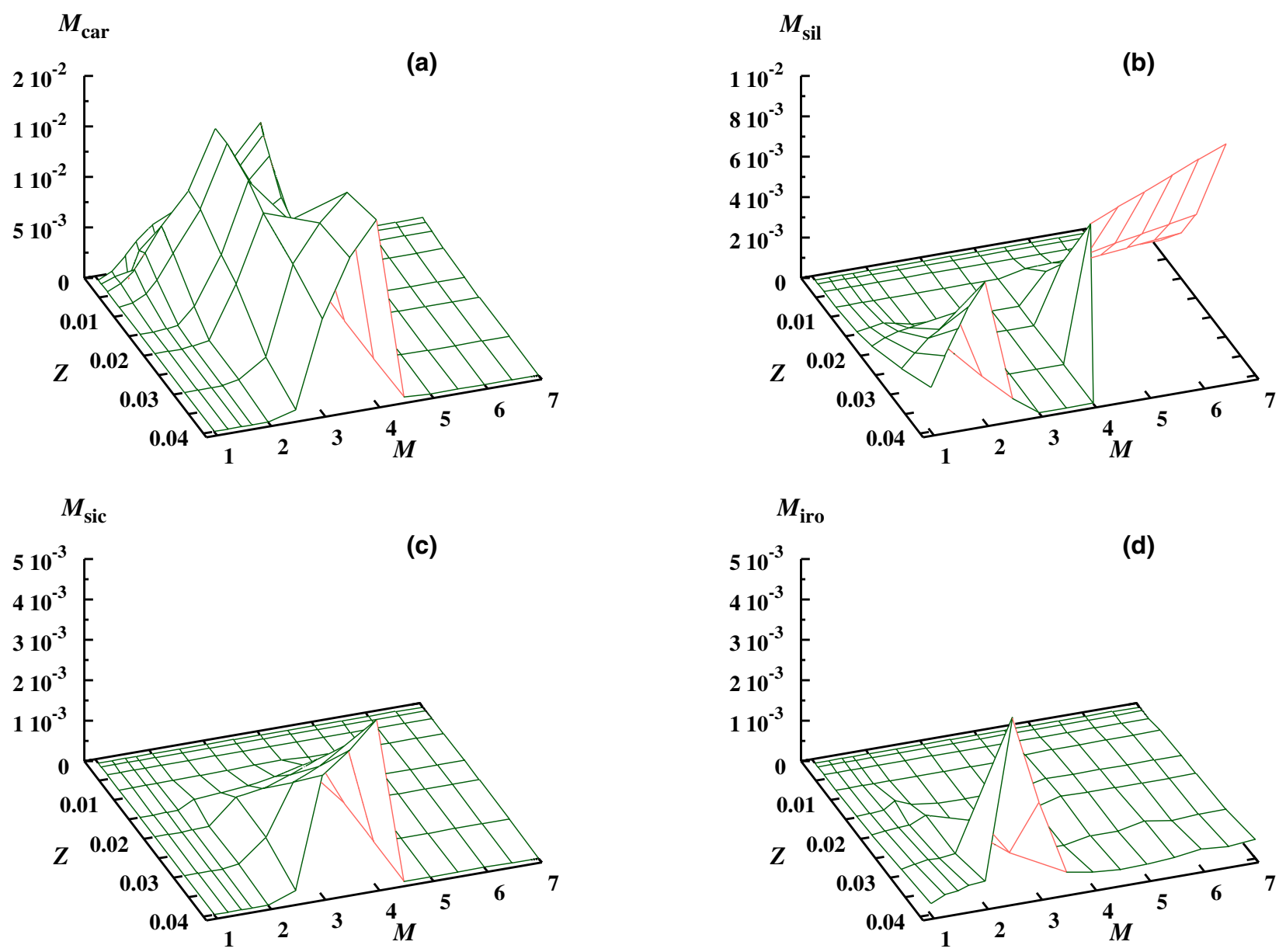

Fig. 10. Dependence of the dust masses returned by single AGB stars for the four main kinds of dust species (silicates, carbon, silicon carbide, and iron) on metallicity $Z$ and initial stellar mass $M_{*}$. All masses are in units of $M_{\odot}$. Data from Ferrarotti \& Gail (2006) with some additional models.

spectroscopic features exist for solid iron. Nevertheless, for reasons of element abundances, it should be an abundantly formed dust species in $\mathrm{S}$ stars and $\mathrm{C}$ stars, and to some extent also in M stars.

$\mathrm{MgS}$ is also observed to be an abundant dust component in many C stars (cf. Molster \& Waters 2003), but it is not included in the modelling, since it is not yet clear by which mechanism it can be formed in a stellar outflow. This, of course, presently prevents its modelling.

Figure 10 shows the calculated dust production rates for the four types of dust considered. In the model calculation of Ferrarotti \& Gail (2006), olivine- and pyroxene-type dust are treated as separate species, but their production rates are added for the reasons mentioned above $\mathrm{e}^{4}$. There is a general tendency for the stars to be a factory either mainly for silicate dust or mainly for carbon dust (cf. Fig. 12 of Ferrarotti \& Gail 2006), because most of the dust formed over the total lifetime of a star on the AGB is formed during the very last pulse cycles on the TP-AGB, where mass-loss rates are highest. If the stars are carbon stars during this phase, they mainly produce carbon dust (and $\mathrm{SiC}$ ); otherwise, they mainly produce silicate dust.

The carbon dust production, shown in Fig. 10a, is dominated by stars with initial masses between about 1.5 and $4 M_{\odot}$ and does not vary much with initial stellar metallicity, since the carbon required for carbon dust production is synthesised from He by the star itself. Stars with initial masses $M>4 M_{\odot}$ also form

\footnotetext{
4 A table with the complete data set showing the separate dust species is added as on-line material to this paper.
}

some carbon dust, but only small amounts during their very last stage of evolution when hot bottom burning is no longer active. Stars with initial masses $M<1.5 M_{\odot}$ do not form much carbon dust because the total mass returned by them on the AGB is quite small or because they do not suffer third dredge-up events (for very low initial masses) or are too few of them.

The production of the other dust species by AGB stars strongly depends on their initial metallicity because the required heavy elements - with the possible exception of $\mathrm{Mg}$ - are not fabricated by AGB stars but have to be formed in many preceding stellar generations until their abundances grow to a level where dust formation becomes possible.

Figure 10b shows the silicate dust production by AGB stars. The silicate production is efficient for stars from essentially that range of initial masses where they do not become efficient carbon dust producers; i.e., the main contribution comes from stars with initial masses $M<1.5 M_{\odot}$ or $M>4 M_{\odot}$. But also in the mass range in between, where the stars are efficient carbon dust factories, they produce some silicate dust before they become carbon stars. The silicate dust production starts to become efficient only at rather high metallicities because only then are sufficient amounts of $\mathrm{Si}, \mathrm{Mg}$, and $\mathrm{Fe}$ for silicate formation available in the stellar outflows.

Figure 10c shows the production of silicon carbide dust by AGB stars. This is produced by carbon stars, and therefore its production is limited to the same range of initial masses as for carbon production. The lack of available $\mathrm{Si}$, however, also prevents the formation of much $\mathrm{SiC}$ in low metallicity stars. 
Figure 10d shows the production of iron dust by AGB stars. Iron dust formation seems to be efficient in outflows from AGB stars only at rather high metallicities, which are not encountered in the Milky Way at the solar circle, but only close to its centre.

The dust-mass injection rate of dust species $j$ into the interstellar medium is given by

$R_{j, l, \mathrm{~d}}(t, r)=\int_{M_{\mathrm{l}}}^{M_{\mathrm{WD}}} \mathrm{d} M \Phi(M) \frac{B\left(t_{\mathrm{b}}, r\right)}{M_{\mathrm{av}}} M_{j, \mathrm{ret}}\left(M, Z_{\mathrm{ISM}}\left(t_{\mathrm{b}}, r\right)\right)$.

The index $l$ here refers to AGB stars. The quantity $\Phi$ is the initial mass function, described in Sect. 2.1.4, $M_{\mathrm{av}}$ the average mass of the stars, given by Eq. (6), and $B(t, r)$ and $Z_{\mathrm{ISM}}(t, r)$ are the stellar birthrate and the metallicity of the interstellar medium at instant $t$ and galactocentric radius $r$, respectively. Both quantities are taken from the model calculation for the evolution of the galactic disk (see Figs. 2 and 3). The instant

$t_{\mathrm{b}}=t-\tau\left(M, Z_{\mathrm{ISM}}\left(t_{\mathrm{b}}, r\right)\right)$

is the time of birth of a star ending its life at instant $t$. The quantity $\tau(M, Z)$ is the lifetime of a star with initial mass $M$ and metallicity $Z$. The metallicity of the stars equals the metallicity $Z_{\text {ISM }}$ of the interstellar medium at their birthtime $t_{\mathrm{b}}$, which is taken from our model for the Milky Ways evolution (cf. Fig. 3). Note that $t_{\mathrm{b}}$ is given by a non-linear equation that has to be solved numerically for each $M$ and $t$. Tables for the mass return in the dust species $j$ by AGB stars of different initial masses and metallicities are given in Ferrarotti \& Gail (2006); some additional models have been calculated for the present work. The integration is performed over the initial masses of the stars from the lower limit $M_{1}$, here taken to be $1 M_{\odot}$, and the upper limit $M_{\mathrm{WD}}$ up to which stars evolve into White Dwarfs, here taken to be $8 M_{\odot}$.

\subsection{Dust production by massive stars}

In principle one has four different processes contributing to the dust return by massive stars that finally explode as $\mathrm{SNe}$ :

1. Dust formed in the massive cool stellar winds of Red Supergiants, i.e., massive stars on the Red Giant Branch. This is relevant only for stars from the range of initial masses $8 \lesssim M \lesssim 40 M_{\odot}$, since only stars from this mass range enter the Red Giant stage.

2. Dust formed in massive shells of $1 \ldots 10 M_{\odot}$ ejected by repeated giant eruptions during an LBV-phase, such as observed in $\eta$ Car (cf. Smith \& Owocki 2006). This is relevant only for massive stars from the region of initial masses $M \gtrsim 40 M_{\odot}$ which evolve through a LBV phase. Most of the mass ejected by these very massive stars prior to their $\mathrm{SN}$ explosion seems to be ejected in a few such events (Smith 2006), which are accompanied by copious dust formation.

3. Dust formed in the outflows from Wolf-Rayet stars.

4. Dust formed in the ejected matter after the final supernova explosion.

The dust grains formed in outflows from Red Supergiants and giant eruptions of very massive stars carry isotopic anomalies resulting from hydrogen burning via the CNO-cycle, while the dust grains formed after a supernova explosion show the very different isotopic signatures from heavy-element synthesis. Both types would be clearly distinguishable, if investigated as presolar grains in the laboratory. Both types should be included as separate types of dust in a model calculation.

\subsubsection{Dust formed in pre-supernovae}

The dust formed in stellar winds or ejecta prior to the supernova explosion is later subjected to the shock wave from the $\mathrm{SN}$ explosion. This shock wave destroys the dust in the swept-up material if the expansion velocity exceeds $150 \mathrm{~km} \mathrm{~s}^{-1}$ (e.g. Jones et al. 1996). For a simple estimation of the importance of this process, we consider the case that the blast wave expands into a medium with constant density. At the end of the adiabatic expansion phase, the radius and the velocity of the shocked region are about (e.g. Shull \& Draine 1987)

$R_{\mathrm{sh}}=16.2 E_{51}^{2 / 7} n_{0}^{-3 / 7} \mathrm{pc}$

$V_{\mathrm{sh}}=331 E_{51}^{1 / 14} n_{0}^{1 / 7} \mathrm{~km} \mathrm{~s}^{-1}$.

The transition between the Sedov-Taylor expansion and the subsequent pressure-dominated snowplow phase occurs at

$t_{\mathrm{ST}-\mathrm{PDE}}=1.91 \times 10^{4} E_{51}^{3 / 14} n_{0}^{-4 / 7} \mathrm{yr}$.

Here $E_{51}$ is the explosion energy in units of $10^{51} \mathrm{erg}$ and $n_{0}$ the density of the ambient medium in units of $1 \mathrm{~cm}^{-3}$. Since the shock velocity drops rapidly in the snowplow phase, the dust destruction occurs mainly up to the end of the adiabatic expansion phase given by Eq. (15).

Red Supergiants: First we consider the case of Red Supergiants and let $E_{51}=n_{0}=1$. Typical expansion velocities of stellar winds of supergiants are $v_{\exp }=20 \mathrm{~km} \mathrm{~s}^{-1}$. The wind material requires a time of about $R_{\mathrm{sh}} / v_{\exp }=790000 \mathrm{yrs}$ to expand to the distance $R_{\mathrm{sh}}$. The shock strength then is sufficient to destroy all dust material ejected during a period of $7.9 \times$ $10^{5}-t_{\mathrm{ST}-\mathrm{PDE}} \approx 7.7 \times 10^{5} \mathrm{yrs}$ before the $\mathrm{SN}$ explosion. This is close to the evolution time on the Red Giant branch (e.g. Schaller et al. 1992). The main period for dust formation of such stars, however, is much shorter. Mass-loss rates of supergiants during the phase where they are enshrouded by massive dust shells are of the order of $10^{-4} \ldots 10^{-3} M_{\odot} \mathrm{yr}^{-1}$ (e.g. van Loon et al. 1999), and this phase can last at most about $10^{5} \mathrm{yrs}$; otherwise, the stellar envelope over the He core would be lost completely by the stellar wind prior to explosion, which is not observed for this mass-range.

Hence, all dust formed by Red Supergiants is expected to be destroyed by the shock wave of the subsequent supernova explosion. Even if some dust survives in some cases, Red Supergiants cannot be important sources for interstellar dust.

Luminous Blue Variables: The expansion velocity of the matter from giant eruptions is somewhat higher than for winds of Red Supergiants (cf. Lamers et al. 2001) and may be as high as $100 \mathrm{~km} \mathrm{~s}^{-1}$. Correspondingly, the supernova shock destroys all the dust that was ejected by a giant eruption if the supernova explosion follows within about $2 \times 10^{5}$ yrs after the end of the LBV phase. The LBV phase, however, seems to occur during the first transition from the blue to the red in the Hertzsprung-Russel diagram (Lamers et al. 2001) and is followed by a WR-phase that lasts about $3 \times 10^{5} \ldots 10^{6}$ yrs (Meynet \& Maeder 2005). If the star finally explodes, the velocity of the shock from the SN explosion is already too slow to destroy the dust at the instant when it catches up with the ejected LBV shell. Dust formed in giant eruptions could therefore be an important source of interstellar dust. Unfortunately, however, there is presently not enough information on the dust production by these objects to include them in the model calculation and dust production by LBVs, so they cannot be considered in our present model calculation.

Clearly, the real situation is more complex since a supernova explodes into the matter ejected by the stellar winds of the 
preceding evolutionary stages (cf. Dwarkadas 2006, for a brief discussion), or into the hot bubbles of other supernovae, and the dust ejected by one massive star may be subjected to the SN blast waves of other massive stars from the same stellar cluster. A more detailed investigation of the whole problem is required to determine the survival probability of dust formed by a star prior to its SN explosion.

Wolf-Rayet stars: Dust forming Wolf-Rayet stars are rare, with only about $10 \%$ of all stars in this evolutionary phase (Marchenko \& Moffat 2006), and their lifetime till SN explosion is only $4 \times 10^{5} \mathrm{yr}$ (van der Hucht 2003); i.e., most of the dust formed will be destroyed by the subsequent SN explosion. Therefore we neglect their contribution to dust production by massive stars.

\subsection{Dust return by supernovae}

Unfortunately, it is presently not definitely known which supernovae do form dust and in what quantities. Undoubtedly there is some dust formed by supernovae since presolar dust grains are known that bear the signatures of element synthesis in supernovae. The abundance of X-grains in the population of presolar $\mathrm{SiC}$ grains, however, is small compared to mainstream SiC grains (cf. Hoppe et al. 2000; Nittler \& Alexander 2003), which are thought to come from AGB stars. Dust formation by supernovae, therefore, seems to be an inefficient process. For supernovae of type Ia, observations even seem to indicate that they do not form dust at all (Borkowski et al. 2006). From the theoretical side also, little is known about dust condensation in $\mathrm{SNe}$; only a few model calculations for dust condensation in supernova ejecta are available (Kozasa et al. 1989; Todini \& Ferrara 2001; Nozawa et al. 2003; Schneider et al. 2004), and they are of a very qualitative nature.

Presently there are no reliable models for dust formation in supernovae available on which one can base a modelling of the contribution of supernovae to the interstellar and presolar dust population. Therefore, in the present model calculation we apply the same simplified approach as in Dwek (1998) to account for the contribution of supernovae to the dust production in the Milky Way. The dust return rate is assumed to be given by the total mass return rate of the key element required to form a particular kind of dust ${ }^{5}$ times some efficiency factor $\eta$. This efficiency factor is simply guessed or is estimated from observational quantities. It is assumed that supernovae of type II produce all types of dust considered here. From theoretical considerations, SN Ia may produce some iron dust, therefore we include iron dust from SN Ia in our model.

We therefore use the following production rates for the dust species

$$
\begin{aligned}
& R_{\mathrm{sil}, l, \mathrm{~d}}(t, r)=\eta_{\mathrm{sil,SN} \mathrm{II}} R_{\mathrm{Si}, \mathrm{SN} \text { II }}(t, r) \frac{A_{\mathrm{sil}}}{A_{\mathrm{Si}}} \\
& R_{\mathrm{car}, l, \mathrm{~d}}(t, r)=\eta_{\mathrm{car}, \mathrm{SN} \text { II }} R_{\mathrm{C}, \mathrm{SN} \text { II }}(t, r) \frac{A_{\mathrm{car}}}{A_{\mathrm{C}}} \\
& R_{\mathrm{sic}, l, \mathrm{~d}}(t, r)=\eta_{\mathrm{sic}, \mathrm{SN} \text { II }} R_{\mathrm{Si}, \mathrm{SN} \text { II }}(t, r) \frac{A_{\mathrm{sic}}}{A_{\mathrm{Si}}}
\end{aligned}
$$

\footnotetext{
5 As key element we usually choose the one of all the elements forming the considered dust species, for which the quantity $\epsilon / i$ takes the lowest value. Here $\epsilon$ is the abundance of an element by number relative to $H$, and $i$ is the number of atoms of the element in the chemical formula of the compound. The key element determines the maximum amount of dust material that can be formed for the considered species.
}

Table 5. Characteristic quantities and numerical coefficients used for calculating grain destruction and grain growth ( $\mathrm{SiC}$ does not form in the ISM).

\begin{tabular}{lcccc}
\hline \hline & Silicates & Carbon & Iron & $\mathrm{SiC}$ \\
\hline$\tau_{j, \mathrm{SNR}}(\mathrm{Gyr})$ & 0.4 & 0.6 & 0.6 & 0.6 \\
$\eta_{\mathrm{SN} \mathrm{II}}$ & 0.00035 & 0.15 & 0.001 & 0.0003 \\
$\eta_{\mathrm{SN} \mathrm{Ia}}$ & 0.0 & 0.0 & 0.005 & 0.0 \\
key element & $\mathrm{Si} \mathrm{or} \mathrm{Mg}$ & $\mathrm{C}$ & $\mathrm{Fe}$ & $\mathrm{Si}$ \\
Atomic weight $A$ & 121.41 & 12.01 & 55.85 & \\
bulk density $\rho_{\mathrm{c}}\left(\mathrm{g} \mathrm{cm}^{-3}\right)$ & 3.13 & 2.25 & 7.86 & \\
\hline
\end{tabular}

$R_{\mathrm{iro}, l, \mathrm{~d}}(t, r)=\eta_{\text {iro,SN II }} R_{\mathrm{Fe}, \mathrm{SN} \text { II }}(t, r) \frac{A_{\text {iro }}}{A_{\mathrm{Fe}}}$.

The index $l$ here refers to supernovae of type II, and $R_{\mathrm{Si}, \mathrm{SN} \text { II }}$ is the rate of mass return to the interstellar medium of element $\mathrm{Si}$ by all supernovae of type II, defined by

$$
\begin{aligned}
R_{\mathrm{Si}, \mathrm{SN} I \mathrm{I}}(t, r)=\int_{M_{\mathrm{WD}}}^{M_{\mathrm{u}}} \mathrm{d} M \Phi(M) \frac{B\left(t_{\mathrm{b}}, r\right)}{M_{\mathrm{av}}} \\
\cdot M_{\mathrm{Si}, \mathrm{ret}}\left(M, Z_{\mathrm{ISM}}\left(t_{\mathrm{b}}, r\right)\right) .
\end{aligned}
$$

The quantity $M_{\mathrm{Si}, \mathrm{ret}}(M, Z)$ is the $\mathrm{Si}$ mass returned by a supernova with initial mass $M$ and metallicity $Z$. The integration is from the lower limit $M_{\mathrm{WD}}$ to the upper limit $M_{\mathrm{u}}$, here taken to be $40 M_{\odot}$ since the tables for mass return by supernovae of Woosley \& Weaver (1995) and Nomoto et al. (2006) only extend up to this upper mass. The mass return rates for the other elements are defined correspondingly. The quantities $A_{\text {sil }}, A_{\text {car }}, A_{\text {sic }}, A_{\text {iro }}$ are the atomic weights of the dust species and $A_{\mathrm{Si}}, A_{\mathrm{car}}, A_{\mathrm{Fe}}$ the atomic weights of the key elements.

For supernovae of type Ia, the dust production rate is

$R_{\text {iro, } l, \mathrm{~d}}(t, r)=\eta_{\text {iro,SN Ia }} R_{\mathrm{Fe}, \mathrm{SN} \mathrm{Ia}}(t, r) \frac{A_{\text {iro }}}{A_{\mathrm{Fe}}}$,

where the index $l$ now refers to supernovae of type Ia. The mass return rate for iron is

$R_{\mathrm{Fe}, \mathrm{SN} \mathrm{Ia}}(t, r)=M_{\mathrm{Fe}, \text { ret }} R_{\mathrm{SN} \mathrm{Ia}}(t, r)$,

where $R_{\mathrm{SNIa}}(t, r)$ is the supernova rate of type Ia, which is taken from the galactic evolution model (see Fig. 2), and $M_{\mathrm{Fe}, \text { ret }}$ is the iron mass returned by supernovae of type Ia and is taken from the tables of Iwamoto (1999).

The quantities $\eta_{\text {sil,SN II }}, \ldots, \eta_{\text {iro,SN Ia }}$ are the efficiencies for conversion of the key elements of the different dust species into dust particles, and they refer to the amount of dust injected into the interstellar medium in relation to the total mass of the key element returned to the interstellar medium. The dust first formed in the expanding SN ejecta is later overrun by the reverse shock and part of it is destroyed again (Dwek 2005; Bianchi $\&$ Schneider 2007; Nozawa et al. 2007). The efficiencies $\eta$ as they are defined here consider the dust destruction by the reverse shock and may therefore be significantly smaller than the efficiency of the initial dust condensation.

So far, only a few attempts have been made to estimate the condensation efficiency in SNe by analysing spectroscopic data, resulting in very different dust yields, from only $5 \times 10^{-4}$ to $4 \times 10^{-3}$ for type II SN 1987A (Ercolano et al. 2007) to 0.12 for SN 2003gd (Sugerman et al. 2006). The values of $\eta$ for different types of SNe are still unknown and have to be guessed somehow. The numerical values chosen in this paper are much lower than the values assumed in Dwek (1998) and are given in Table 5 . The values for the efficiencies $\eta_{\text {sil,SN II }}, \eta_{\text {sic,SN II }}$, and 


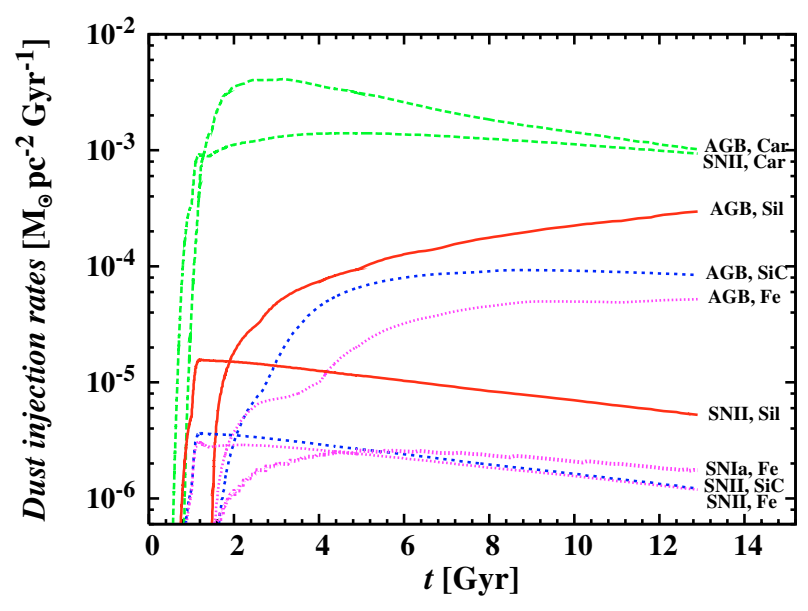

Fig. 11. Evolution of the dust injection rates at the solar circle from different stellar sources.

$\eta_{\text {car,SN II }}$ of silicate, $\mathrm{SiC}$, and carbon dust formation in SN II, respectively, are estimated from the abundances of presolar silicate grains from supernovae, of X-type SiC grains, and graphite grains from supernovae. This is discussed in Sect. 5.3. The efficiencies $\eta_{\text {iro,SN II }}$ and $\eta_{\text {iro,SN Ia }}$ for iron dust production in SNe of type II and type Ia, respectively, are arbitrarily set to a low nonzero value, but they may well be equal to zero. Tests run without SN Ia dust showed no influence on the amount of iron dust from molecular clouds, since stardust is only important as seed grains for the ISM dust production at an early time.

\subsection{Dust injection rates for the Milky Way model}

Figure 11 shows the variation with time of the dust injection rates from stellar sources into the interstellar medium at the solar circle, as calculated for our model of the evolution of the Milky Way. The dust injection rate in this model is dominated by carbon dust from AGB stars and $\mathrm{SNe}$ and by silicate dust from AGB stars, except for the very first period before the first appearance of AGB stars, where dust return from SNe dominates. The SN injection rates are very uncertain, however, since they depend on the efficiencies $\eta$, which are only badly known and in this paper are determined from abundance ratios of presolar dust grains from AGB stars and supernovae.

\section{Dust evolution in the ISM}

\subsection{Dust destruction in the interstellar medium}

The analysis of astronomical observations and presolar dust grains from meteorites indicates a high degree of dust processing in the ISM. The processes can be divided into two groups:

1. the destruction processes (thermal sputtering, evaporation in high-velocity grain-grain collisions, chemical sputtering) and the growth by accretion that changes the total dust mass;

2 . the processes modifying the grain size distribution or the phase of the grains (shattering in grain-grain collisions, coagulation, amortisation of crystalline stardust, etc.).

Detailed discussions of dust processing in the ISM can be found in Tielens (2005), for example. Since in this paper we do not study the grain size distribution, we are interested in the first group of processes only, i.e., in the destruction processes and the growth processes in the ISM.
The dominant dust destruction process is dust destruction in the ISM by sputtering in high-velocity $\mathrm{SN}$ shocks $(v>$ $150 \mathrm{~km} \mathrm{~s}^{-1}$ ) (cf. Jones et al. 1996), which results in injection of atoms into the gas phase by interaction with impinging energetic ions. This process works almost exclusively in the warm phase of the interstellar medium, which links in principle the dust destruction problem in the ISM closely to the multiphase structure of the ISM. Since we approximate the ISM in our present model by a simple one-phase model, we describe this process in the approximation of Jones et al. (1996) that describes the destruction process in terms of grain lifetimes against destruction by $\mathrm{SN}$ remnants $\tau_{j, \mathrm{SNR}}$. The change in surface density of the dust species of kind $j$ per unit time by dust destruction is

$L_{j, \mathrm{~d}}=-\frac{\Sigma_{j, \mathrm{~d}}}{\tau_{j, \mathrm{SNR}}}$.

Since we only consider equations for the total element abundances (gas+dust) in the ISM and no separate equations for the gas-phase abundance, the mass return to the gas phase needs no special treatment. This is automatically accounted for if the gas phase abundances are calculated from Eqs. (10).

The grain lifetime at any radius $r$ and instant $t$ can be expressed by the current lifetime at the solar circle according to Dwek (1998) by the approximate scaling law:

$\tau_{j, \mathrm{SNR}}(t, r)=\frac{\Sigma_{\mathrm{ISM}}(t, r)}{\sum_{\mathrm{ISM}}\left(t_{\mathrm{G}}, r_{\odot}\right)} \frac{R_{\mathrm{SN}}\left(t_{\mathrm{G}}, r_{\odot}\right)}{R_{\mathrm{SN}}(t, r)} \tau_{j, \mathrm{SNR}}\left(t_{\mathrm{G}}, r_{\odot}\right)$.

The total SN rate $R_{\mathrm{SN}}(t, r)$ can be replaced by the stellar birthrate $B(t, r)$ with the assumption of the spatial and temporal constancy of the IMF. The dust species considered in our model are listed in Table 4. For these dust species, we need to know the destruction timescales $\tau_{j, \mathrm{SNR}}(t, r)$.

For the current grain lifetimes at the solar circle against destruction in all phases of the ISM, we take the theoretical estimates (Jones et al. 1994, 1996, see also the references therein) of 0.6 and $0.4 \mathrm{Gyr}$ for carbonaceous and silicate dust, respectively. Unfortunately they did not present the corresponding timescales for iron and silicon carbide dust, but their results for the sputtered dust mass fraction for different shock velocities for iron and for silicon carbide dust are somewhat higher but similar to carbon dust. We therefore choose for both the same lifetime of $0.6 \mathrm{Gyr}$ as for carbon dust. The values of $\tau_{j, \mathrm{SNR}}\left(t_{\mathrm{G}}, r_{\odot}\right)$ used in the model calculation are also shown in Table 5.

\subsubsection{Evolution of stardust}

Stardust is only destroyed in the ISM and does not gain mass by accretion of gas phase material. All material from such grains ejected into the gas phase rapidly mixes with the existing ISM gas-phase material, and the specific isotopic anomalies carried by the stardust material are lost by mixing together eroded material of grains from many different kinds of stellar sources. If such material is later accreted by dust grains in the ISM, it shows no isotopic anomalies. Even if such material grows as mantle material on stardust cores, the differences in isotopic composition between core and mantle survive since dust grains in the ISM are not expected to ever become hot enough (>1000 K) for long enough periods for solid state diffusion to smooth out isotopic abundance differences between a stardust core and an ISM-grown mantle; hence, any accreted mantle material can be clearly distinguished (if it could be analysed in the laboratory) from cores originating from stellar sources by showing isotopic 
abundances ratios close to Solar System isotopic abundance ratios, even if the general chemical composition and mineralogical structure of an ISM-grown mantle material should resemble that of a core with a stellar origin. Therefore we treat the dust species from stellar sources in our model as separate dust components and omit the growth term for these species in Eq. (9); only the destruction and source terms are retained.

\subsection{Dust growth in the interstellar medium}

Dust grains cycle between the cloud and intercloud phase of the ISM on at timescale of $\simeq 3 \times 10^{7} \mathrm{yr}$ (e.g. Draine 1990; Tielens 1998), undergoing destruction in the warm intercloud medium. All theoretical calculations of grain lifetimes against destruction by SN shocks agree that they are much shorter than the $\sim 2$ Gyr timescale of dust injection by stars (e.g. Jones et al. 1996; Tielens et al. 2005). This requires an efficient mechanism of replenishment of the dust content of the ISM. Another proof of dust growth in the ISM is that gas abundances in the ISM of major dust-forming elements show strong depletion in comparison to solar abundances (e.g. Savage \& Sembach 1996; Jenkins 2004), which correlates with the ISM density. Also, the high dust content observed in some high-redshift objects seems to require dust growth in the interstellar medium (Dwek et al. 2007). The only possible site of grain growth in the ISM is the dense molecular clouds of the cold phase of the ISM (Draine 1990).

It is known that the density of the ISM is not high enough to allow for the formation of new dust grains, only low temperature accretion of refractory material on pre-existing stellar grains is possible. The thin mantles accreted in the ISM are likely to be more volatile than stellar dust and can be lost more readily during dust cycling between ISM phases. Besides, in dense molecular clouds the accretion will be faster, and grains will probably be formed far from equilibrium, so that one would expect the grain mantles to be amorphous and heterogeneous (Jones 2005). Thus, dust accreted in molecular clouds (the MC-grown dust) has different properties from stardust and is treated in our model as a separate component denoted by an index ISM.

Dust growth in molecular clouds by accretion on existing grains needs to be considered in our model for silicate and carbon dust, since it is known that observed depletions of the elements in the ISM are too high as that only destruction can be active (cf. Jenkins 2004, for a recent discussion). The grains that serve as growth centres for accretion of gas-phase material need not necessarily be the stardust particles, though these are needed to serve as initial growth centres for a start-up of the whole process. Also, fragments formed from shattering of MC-grown grains by SN shock waves in the warm component of the ISM may serve as growth centres for accretion of refractory elements in the gas phase if mixed into molecular cloud cores.

An unclear case for growth in the ISM is iron dust, which might be a component of the ISM dust mixture, if not all $\mathrm{Fe}$ is used up by the formation of magnesium-iron-silicates. However, metallic iron is probably unstable against oxidation in the ISM (Jones 1990), while, on the other hand, iron oxides do not seem to form a significant species in the ISM dust mixture (Chiar \& Tielens 2006). We consider iron in our model calculation as a possible MC-grown dust component since there are observational indications that not all condensed iron always resides in silicates (cf. Cartledge et al. 2006, their Fig. 10).

How the growth process works in detail is not definitely known. For interstellar carbon dust, it may proceed in the way described in Jenniskens et al. (1993) as a multistep process, initiated by deposition of ice mantles, and proceeding via canonisation and polymerisation driven by UV irradiation. The problem of growth of silicate dust in the ISM has long remained unsolved, because the formation of tetrahedral $\mathrm{SiO}_{4}$-structures probably requires higher temperatures than the 10-30 K observed in molecular clouds. At these low temperatures, ice mantles are likely formed on the grain surface, preventing further growth of silicates. The solution of the problem is possibly provided by intermittent dissipation of turbulence in molecular clouds (Falgarone et al. 2006). The large local release of nonthermal energy in the gas by short bursts of turbulent dissipation has been shown to be able to trigger a specific warm chemistry, which can be traced by the high abundances of $\mathrm{CH}^{+}, \mathrm{H}_{2} \mathrm{O}$, and $\mathrm{HCO}^{+}$observed in diffuse gas. It is shown that signatures of warm chemistry survive in the gas more then $10^{3} \mathrm{yr}$ during chemical and thermal relaxation phases, see Fig. 10 in Falgarone et al. (2006). Such a local change in the gas temperature could provide the mechanism for further silicate growth, if the grain temperature increases enough for ice mantles to evaporate. The latter is defined by equating energy from collisions with warm gas and the emitted infrared energy, and thus depends on infrared absorption coefficients of mantle and core grain material; e.g. it differs noticeably for water and organic ices.

Our preliminary estimates show that the energy released locally by turbulent dissipation in molecular clouds is sufficient to evaporate organic ice mantles from the surface of silicate grains, although detailed calculations of temperatures and residence time in the relaxation phase for grains with different compositions have to be done to make quantitative estimates. This is a separate problem that is important for understanding the physics of dust growth in molecular clouds, and will be studied in further papers.

It is assumed in the following that the silicate and carbon dust grains grow as separate species. In molecular clouds the growth of ice mantles certainly does not distinguish between carbon and silicon-bearing gas-phase species, and the mantles probably have a mixed chemistry. It is presently not known how it is possible to form either carbon or silicate dust grains under these circumstances, and there is the possibility that carbon grains grow silicate coatings and silicate grain grow carbonaceous coatings. We do not consider these possible complications here.

\subsubsection{Growth of dust grains in molecular clouds}

In calculating the growth rates for the dust species, we follow a different procedure, as in Dwek (1998). Some modifications are necessary because (1) we wish to consider specific dust components and not merely the surface density of dust forming elements residing in some not closer specified dust components, and (2) since it is assumed that growth of dust is essentially restricted to molecular clouds (cf. Draine 1990) which relates the dust growth problem, like the dust destruction problem, closely to the multiphase structure of the ISM, which has approximately to be taken into account (for a different type of approach than in this paper see Liffman \& Clayton 1989).

It is generally assumed (i) that the growth of dust grains of a specific kind $j$ is governed by some rate determining reaction step, usually by adding of that one of the elements required to form the chemical compound that has the lowest abundance in the gas phase, and (ii) that the rate of adding of all other more abundant elements adapts to the slowest process. The growth is determined in this case by some specific key element and a special atomic or molecular species from the gas phase carrying most or all of this key element, the growth species. The key 
elements for the condensed phases of interest are given in Table 5. The equation for the change in the mass $m_{j}$ of a single grain of species $j$ is

$\frac{\mathrm{d} m_{j}}{\mathrm{~d} t}=\mathcal{A} A_{j} m_{\mathrm{AMU}} \frac{v_{j, \mathrm{~m}}}{v_{j, \mathrm{c}}} \alpha_{j} v_{j, \mathrm{th}, \mathrm{gr}} n_{j, \mathrm{gr}}$.

Here $n_{j, \mathrm{gr}}$ is the particle density of the growth species, $v_{j \text {,th,gr }}$ its thermal velocity, $\alpha_{j}$ the growth coefficient, $\mathcal{A}$ the surface area of the grain, and $A_{j}$ the atomic weight of one formula unit of the dust material under consideration. Also $v_{j, \mathrm{~m}}$ and $v_{j, \mathrm{c}}$ are the number of atoms of the key element contained in the growth species and in the formula unit of the condensed phase, respectively. Evaporation is neglected since this is not important at the low temperatures in molecular clouds. The change in the mass density $\rho_{j}$ of the dust particles in a molecular cloud is obtained by multiplying the growth equation of single grains by the number density of grains and the probability distribution of grain radii (assuming spherical grains) and integrating over all grain radii $a$. One obtains

$\frac{\mathrm{d} \rho_{j}}{\mathrm{~d} t}=\alpha_{j} v_{j, \mathrm{th}, \mathrm{gr}} n_{j, \mathrm{gr}} \frac{3 V_{1, j}\left\langle a^{2}\right\rangle}{\left\langle a^{3}\right\rangle} \frac{v_{j, \mathrm{~m}}}{v_{j, \mathrm{c}}} \rho_{j}$,

where $V_{1, j}=A_{j} m_{\mathrm{AMU}} / \rho_{\mathrm{c}}$ is the volume of one formula unit in the condensed phase, $\rho_{\mathrm{c}}$ is the mass density of the condensed phase, and $\langle\ldots\rangle$ denotes averaging with respect to the size distribution of grains. We define the following average grain radius

$\langle a\rangle_{3}=\frac{\left\langle a^{3}\right\rangle}{\left\langle a^{2}\right\rangle}$.

For a MRN size distribution (Mathis et al. 1977), we have for instance

$\langle a\rangle_{3}=\sqrt{a_{0} a_{1}} \approx 0.035 \mu \mathrm{m}$,

where $a_{0}=0.005 \mu \mathrm{m}$ and $a_{1}=0.25 \mu \mathrm{m}$ are the lower and upper limits of the distribution of grain radii, respectively.

The maximum possible particle density of the growth species is

$n_{j, \mathrm{gr}, \max }=\frac{N_{\mathrm{H}} \epsilon_{j}}{v_{j, \mathrm{~m}}}$,

where $N_{\mathrm{H}}$ is the number density of $\mathrm{H}$ nuclei in the molecular cloud (usually equal to $2 n_{\mathrm{H}_{2}}$ ), and $\epsilon$ the element abundance of the key element, possibly lowered by the fraction of this element that is blocked in some unreactive molecular species. Let $f$ denote the fraction of the key element already bound in the dust species under consideration, the degree of condensation $f$. The gas-phase density of the growth species is $(1-f) n_{j, \text { gr,max }}$. Hence we obtain the following equation for the degree of condensation in the molecular cloud

$\frac{\mathrm{d} f}{\mathrm{~d} t}=\frac{1}{\tau_{j, \mathrm{gr}}} f(1-f)$,

with

$\frac{1}{\tau_{j, \mathrm{gr}}}=\alpha_{j} v_{j, \mathrm{th}, \mathrm{gr}} \frac{3 V_{1, j}}{\langle a\rangle_{3}} \frac{\epsilon}{v_{j, \mathrm{c}}} N_{\mathrm{H}}$.

Numerically, we have

$$
\begin{aligned}
\tau_{j, \mathrm{gr}}= & 46 \mathrm{Myr} \\
& \times \frac{v_{j, \mathrm{c}} A_{j, \mathrm{~m}}^{\frac{1}{2}}}{A_{j, \mathrm{c}}}\left(\frac{\rho_{\mathrm{c}}}{3 \mathrm{~g} \mathrm{~cm}^{-3}}\right)\left(\frac{3.5 \times 10^{-5}}{\epsilon}\right)\left(\frac{10^{3} \mathrm{~cm}^{-3}}{N_{\mathrm{H}}}\right),
\end{aligned}
$$

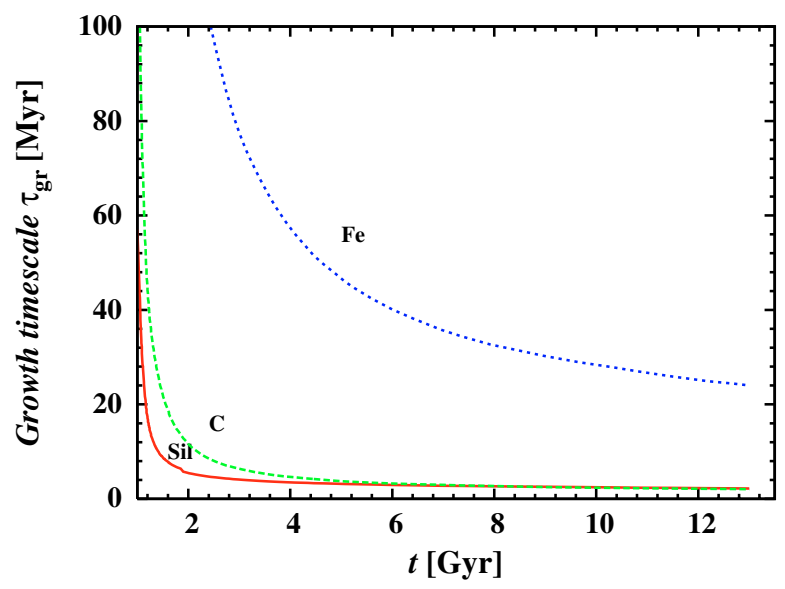

Fig. 12. Growth timescale for the dust species growing in molecular clouds for the Milky Way model at the solar circle: silicate dust (full line), carbon dust (dashed line), iron dust (dotted line).

where $\tau_{j, \mathrm{gr}}$ is evaluated with characteristic values for the physical variables. The temperature of clouds is assumed to be $10 \mathrm{~K}$, and the growth coefficient $\alpha$ at such low temperatures is assumed to be $\alpha=1$. The characteristic growth time is generally short compared to the lifetime of molecular clouds, except for very low metallicity of the ISM. As an example, Fig. 12 shows the growth timescale $\tau_{j, \text { gr }}$ calculated from our Milky Way model at the solar circle for the important dust species.

In principle, the average grain radius $\langle a\rangle_{3}$ depends on the degree of condensation $f$ ( $a \propto f^{1 / 3}$ for compact structures), but we neglect this weak dependence. In this case the equation for $f$ can immediately be integrated with the result

$f(t)=\frac{f_{0} \mathrm{e}^{t / \tau_{\mathrm{gr}}}}{1-f_{0}+f_{0} \mathrm{e}^{t / \tau_{\mathrm{gr}}}}$.

Here $f_{0}$ is the initial degree of condensation at $t=0$. For $t \gg \tau_{\mathrm{gr}}$ the degree of condensation approaches $f=1$.

\subsubsection{Source term for dust production}

Molecular clouds form in the interstellar medium by instabilities, mainly during the compression of ISM material in the snowplow phase of SN shocks. They disappear within a rather short time if active star formation starts and winds of massive stars and expanding supernova bubbles disperse the clouds. For the average lifetime of molecular clouds, we take an observationally and theoretically motivated value of $\approx 1 \times 10^{7}$ yrs (Leisawitz et al. 1989; Williams \& McKee 1997; Matzner 2002; Krumholz $\&$ McKee 2006; Blitz et al. 2007). This value for the lifetime is somewhat shorter than used in Tielens (1998) in his model of dust growth in clouds, but seems to be more appropriate for the most massive clouds, which contain nearly all of the ISM mass in clouds. The lifetimes of the clouds equals the characteristic timescale $\tau_{\text {exch }}$ by which matter is exchanged between clouds and the remaining ISM.

At the instant of cloud formation, the clouds inherit the dust content of the interstellar medium outside of clouds. The dust content of the matter outside of dense clouds is lower than within clouds, since dust destruction processes operate in this material, while in clouds the dust grows by accreting not yet condensed refractory elements. In fact, except if the metallicity of the ISM is very low, the growth timescale is much shorter than the lifetime 
of the cloud, and the condensation of the refractory elements runs into completion before the cloud disappears.

Let the initial degree of condensation of the key element for some dust species be $f_{0}$. If after a period $t$ a cloud is rapidly dispersed, the degree of condensation in the matter returned to the ISM material outside clouds is equal to the value given by Eq. (32). The effective dust mass return for species $j$ by a molecular cloud is then

$M_{j, \mathrm{~d}, \text { cloud }}=\left(f(t)-f_{0}\right) X_{j, \max } M_{\text {cloud }}$,

where

$X_{j, \text { max }}=\frac{A_{j} \epsilon}{\left(1+4 \epsilon_{\mathrm{He}}\right) v_{j, \mathrm{c}}}$

is the maximum possible mass fraction of the dust species in the material of the molecular cloud and $M_{\text {cloud }}$ is the cloud mass. In principle, one has to observe that some fraction of the cloud mass is converted into stars and not converted into other phases of the ISM. Since we describe the effect of dust consumption by star formation within the frame of our approximation by a separate term in Eq. (9), this process does not need to be accounted for in Eq. (33).

Equation (33) has to be multiplied by the probability $P(t)$ that the cloud is destroyed at some instant within the period between $t$ and $t+\mathrm{d} t$,

$P(t)=\frac{1}{\tau_{\text {exch }}} \mathrm{e}^{-t / \tau_{\text {exch }}}$,

and integrated over $t$. Here it is assumed that the cloud destruction occurs at random with a mean lifetime $\tau_{\text {exch }}$. Finally, averaging with respect to the mass spectrum of clouds and multiplying with the surface number density of clouds, one obtains for the mass return rate of $\mathrm{MC}$-grown dust per unit time and unit area of the galactic disk

$G_{j, \mathrm{~d}}=\frac{1}{\tau_{\mathrm{exch}}}\left(f_{j, \mathrm{ret}}-f_{j, 0}\right) X_{j, \max } \Sigma_{\mathrm{cloud}}$

where $\Sigma_{\text {cloud }}$ is the surface mass density of clouds, and the average degree of condensation on cloud dispersal is

$f_{j, \text { ret }}(t)=\frac{1}{\tau_{\text {exch }}} \int_{0}^{t} \mathrm{~d} x \mathrm{e}^{-x / \tau_{\text {exch }}} \frac{f_{j, 0}(t-x) \mathrm{e}^{x / \tau_{j, g r}}}{1-f_{j, 0}(t-x)\left(1-\mathrm{e}^{x / \tau_{j, \mathrm{gr}}}\right)}$.

The quantity $G_{j, \mathrm{~d}}$ is the gain term that has to be used in Eq. (9) for the evolution of the MC-grown dust component $j$.

In principle, the evaluation of this term requires considering a multiphase ISM where molecular clouds form one of the components. Since we wish to consider the simpler model of a one-phase ISM, we have to cast Eq. (36) in an appropriate form for this case. In terms of the mass fraction of clouds in the ISM $X_{\text {cloud }}=\Sigma_{\text {cloud }} / \Sigma_{\text {ISM }}$, we have

$G_{j, \mathrm{~d}}=\frac{X_{\text {cloud }}}{\tau_{\text {exch }}}\left(f_{j, \text { ret }} \Sigma_{j, \mathrm{~d}, \max }-\tilde{X}_{j, \mathrm{~d}} \Sigma_{\mathrm{ISM}}\right)$,

where

$\Sigma_{j, \mathrm{~d}, \max }=\frac{A_{j}}{v_{i, j} A_{i}} \Sigma_{i}$

is the maximum possible surface density of dust of kind $j$ if all material from the ISM that can be condensed into this dust species is really condensed, and $\tilde{X}_{j, \mathrm{~d}}$ is the mass fraction of dust of kind $j$ in that part of the ISM that is not in clouds. Here $\Sigma_{i}$ is the surface mass density of the key element for species $j$ in the ISM. For the average mass-fraction of dust in the total ISM, we have

$X_{j, \mathrm{~d}}=\tilde{X}_{j, \mathrm{~d}}\left(1-X_{\text {cloud }}\right)+f_{j, \text { ret }} X_{j, \mathrm{~d}, \max } X_{\text {cloud }}$,

which yields

$\tilde{X}_{j, \mathrm{~d}} \Sigma_{\mathrm{ISM}}=\frac{1}{1-X_{\text {cloud }}} \Sigma_{j, \mathrm{~d}}-\frac{X_{\text {cloud }}}{1-X_{\text {cloud }}} f_{j, \text { ret }} \Sigma_{j, \mathrm{~d}, \text { max }}$.

It follows that

$G_{j, \mathrm{~d}}=\frac{X_{\text {cloud }}}{\tau_{\text {exch }}\left(1-X_{\text {cloud }}\right)}\left[f_{j, \text { ret }} \Sigma_{j, \mathrm{~d}, \max }-\Sigma_{j, \mathrm{~d}}\right]$.

We define the effective exchange time

$\tau_{\text {exch,eff }}=\tau_{\text {exch }} \frac{1-X_{\text {cloud }}}{X_{\text {cloud }}}$.

This is much longer than $\tau_{\text {exch }}$, since $X_{\text {cloud }} \ll 1$, and reflects the fact that it requires many timescales $\tau_{\text {exch }}$ to cycle all ISM material through clouds where it is laden with fresh dust. Our final result for the dust production term is

$G_{j, \mathrm{~d}}=\frac{1}{\tau_{\text {exch,eff }}}\left[f_{j, \mathrm{ret}} \Sigma_{j, \mathrm{~d}, \max }-\Sigma_{j, \mathrm{~d}}\right]$

This is the appropriate dust production term for MC-grown dust in the approximation of a one-phase ISM model. In this model the mass-fraction $X_{\text {cloud }}$ of the ISM in clouds is a free parameter that does not follow from the model calculation but has to be taken from observations. We will use a value of $X_{\text {cloud }}=0.2$ (cf. Tielens 2005), which is appropriate for the ISM at the solar circle.

\subsubsection{Limit cases}

The degree of condensation $f_{j \text {,ret }}$ in the material returned from clouds at the time of their dispersal essentially depends on the ratio of the growth timescale $\tau_{\mathrm{gr}}$ to the average cloud lifetime $\tau_{\text {exch }}$. If $\tau_{\mathrm{gr}} \gg \tau_{\text {exch }}$ (slow growth at low metallicities) one expects that only small amounts of dust are added to the initial dust content; in the opposite case (rapid growth at normal metallicities), one expects complete condensation in the returned material. This can be confirmed by calculating the lowest order terms of a series expansion of the integral in Eq. (37) for the two limiting cases. In the following we assume that $t \gg \max \left(\tau_{\operatorname{exch}}, \tau_{j, \mathrm{gr}}\right)$ so that the upper limit of integration in Eq. (37) can be replaced by $\infty$ and so $f_{j, 0}$ is essentially constant over timescales of the order of $t \gg \max \left(\tau_{\text {exch }}, \tau_{j, \text { gr }}\right)$.

For slow growth $\left(\tau_{\mathrm{gr}} \gg \tau_{\text {exch }}\right)$ one introduces $t / \tau_{\text {exch }}$ as integration variable, expands $\exp \left[\left(\tau_{\operatorname{exch}} / \tau_{\mathrm{gr}}\right) t\right]$ in a series, and integrates term-by-term. The result is in the linear approximation

$f_{j, \text { ret }} \approx f_{0}\left(1+\frac{\tau_{\text {exch }}}{\tau_{\mathrm{gr}}}\right)$.

If this is inserted into Eq. (36) one recognises that $\tau_{\text {exch }}$ cancels out and, thus, the amount of dust produced during the residence time of ISM material in the cloud phase is determined by the details of the growth process.

For rapid growth $\left(\tau_{\text {exch }} \gg \tau_{\text {gr }}\right)$, one introduces $t / \tau_{\text {gr }}$ as the integration variable, expands $\exp \left[\left(\tau_{\mathrm{gr}} / \tau_{\mathrm{exch}}\right) t\right]$ in a series, and integrates term-by-term. The result is in the linear approximation

$f_{j, \text { ret }} \approx 1+\ln f_{0} \frac{\tau_{\mathrm{gr}}}{\tau_{\mathrm{exch}}}$ 


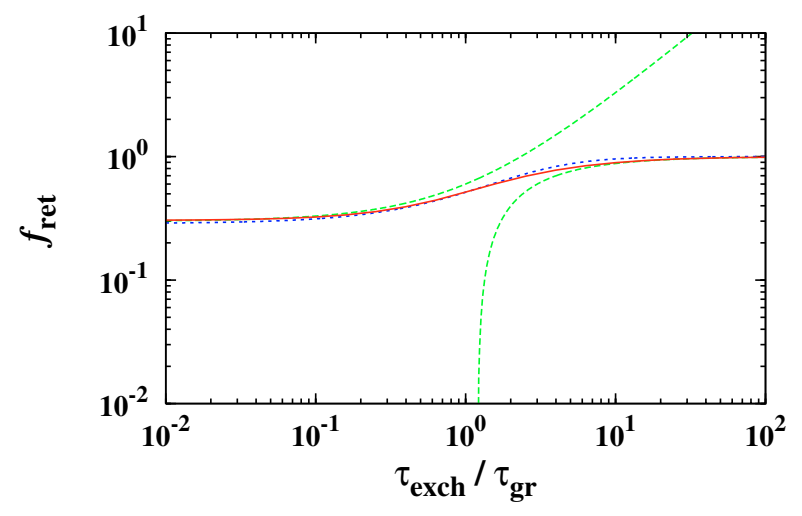

Fig. 13. Approximation for the variation of the degree of condensation $f_{\text {ret }}$ with $\tau_{\text {gr }} / \tau_{\text {exch }}$ for $f_{0}=0.3$. The full line shows the result of a numerical evaluation of the integral (37), the dashed lines the two limit cases Eqs. (43) and (44), and the dotted line the approximation (45) (the full and dashed lines nearly coincide).

If this is inserted into Eq. (36), one finds that the dust production by the clouds is nearly independent of the details of the growth process within the clouds and is (almost) exclusively determined by the cycling frequency of ISM material between the clouds and the other phases of the ISM. The composition of the dust, of course, is determined in this case by the details of growth processes.

The variation in $f_{j \text {,ret }}$ with $\tau_{\text {gr }} / \tau_{\text {exch }}$ in both limit cases for a value of $f_{0}=0.3$ are shown in Fig. 13 with the result of a numerical evaluation of the integral (37). A rather accurate analytic fit formula for the full range of $\tau_{\mathrm{gr}} / \tau_{\text {exch }}$ values is

$f_{j, \mathrm{ret}}=\left(\frac{1}{f_{j, 0}^{2}\left(1+\left(\tau_{\mathrm{exch}} / \tau_{\mathrm{gr}}\right)\right)^{2}}+1\right)^{-1 / 2}$.

The results for other values of $f_{0}$ are similar. Only for very small $f_{0}$ does the approximation becomes somewhat worse in the transition region $\tau_{\mathrm{gr}} / \tau_{\mathrm{exch}} \approx 1$, but for bigger $f_{0}$ it improves. For the purpose of model calculations, it suffices to use the approximation (45).

\subsection{The individual dust species}

Evaluation of the source term Eq. (42) for dust requires calculating the growth time scale $\tau_{j, \text { gr }}$, given by Eq. (30), $X_{j \text {, max }}$ given by Eq. (34), and the degree of condensation $f_{j, \text { ret }}$ in the returned material, which we calculate from the approximation (45), for all dust species $j$ which are formed by growth in molecular clouds.

The constants required for calculating these quantities are given in Table 5. The growth coefficient is assumed to be $\alpha=1$ for all cases since, at the low temperatures in dense molecular clouds of about $10 \mathrm{~K}$, even the weak attractive van der Waals forces lead to adsorption. The basic theory for this is discussed, e.g., in Hollenbach \& Salpeter (1970), and Watson (1975).

For calculating the average $\langle a\rangle_{3}$, we use in all cases the approximation Eq. (28) following from a MRN-size distribution (Mathis et al. 1977). This is only a crude approximation; but without attempting to calculate grain size distributions, it is hardly possible to fix this quantity with more accuracy.
The initial value $f_{j, 0}$ for calculating $f_{j, \text { ret }}$ is given by the degree of condensation in that part of the ISM matter that is not in clouds, i.e., one has

$f_{j, 0}=\frac{\tilde{X}_{j, \mathrm{~d}}}{X_{j, \max }}$

Using this and Eqs. (45) in (39) yields a non-linear equation that has to be solved for $f_{j, 0}$. For most purposes it suffices to replace the value of $f_{j, 0}$ by the approximation $f_{j, 0} \approx X_{j, \mathrm{~d}} / X_{j, \max }$ for solving Eq. (39) since the difference between $\tilde{X}_{j, \mathrm{~d}}$ and $X_{j, \mathrm{~d}}$ is not very big.

\subsubsection{Silicates}

The silicate dust in the ISM accounts for about one half of the total dust mass (e.g. Dwek 2005), but its composition is still a matter of debate. Studies of silicate composition based on interstellar depletions, modelling of extinction curve, and in situ measurements of dust in the local ISM give quite different results, although they all agree on olivine $\left(\left[\mathrm{Mg}_{x} \mathrm{Fe}_{1-\mathrm{x}}\right]_{2} \mathrm{SiO}_{4}\right.$ with $0<x<1)$ and pyroxene $\left(\mathrm{Mg}_{\mathrm{x}} \mathrm{Fe}_{1-\mathrm{x}} \mathrm{SiO}_{3}\right.$ with $\left.0<x<1\right)$ as major candidates for ISM silicates. A number of studies of depletions of $\mathrm{Mg}, \mathrm{Fe}, \mathrm{Si}$, and $\mathrm{O}$ atoms in the interstellar gas phase came to the conclusion that observed depletions indicate an olivine-type stoichiometry of dust in the diffuse ISM (Savage \& Sembach 1996; Jones 2000). A recent attempt to fit the silicate features of the interstellar extinction curve Min et al. (2007) found that the composition of the ISM silicates is consistent with a Mg-rich mixture of olivine and pyroxene with a bigger contribution from pyroxene than from olivine. Fitting of the 9 and $18 \mu \mathrm{m}$ features of the extinction curve shows that, while the $9 \mu \mathrm{m}$ feature can be fitted well by olivine dust, the position and peak strength of $18 \mu \mathrm{m}$ feature is fitted much better with a pyroxenetype stoichiometry (Demyk 1999).

An olivine-pyroxene mixture with a contribution of more pyroxene than olivine is therefore chosen for modelling the ISM silicates in the present paper. As a first approximation we adopt a fixed silicate composition to study silicate dust production by dust growth in molecular clouds. Modelling a variable silicate composition, depending on local growth conditions, is a challenge to be considered in future papers. Let $f_{\mathrm{ol}}$ be the (fixed) fraction of the silicate dust that has olivine stoichiometry; the fraction $1-f_{\mathrm{ol}}$ then has pyroxene stoichiometry. Assuming the same $\mathrm{Mg}$ fraction $x$ for both olivine and pyroxene in our model, two parameters determine the silicate properties: $f_{\mathrm{ol}}$ and $x$.

The total efficiency of dust production by molecular clouds does not show a significant dependence on the choice of the parameters $f_{\mathrm{ol}}$ and $x$. Variations of the Mg-fraction $x$ change the total dust mass on the level of $10 \%$ at most, but define the silicate-to-iron dust mass ratio. This is due to the fact that for the $\mathrm{Mg}$-rich mixtures that are considered here, $\mathrm{Mg}$ is the critical growth species. With decreasing $x$, less $\mathrm{Mg}$ is needed for silicate dust growth, but the total silicate mass increases due to a bigger contribution from the Fe-bearing component, while at the same time less Fe remains for the growth of solid iron. We fix the $\mathrm{Mg}$ fraction $x$ to a value of $x=0.8$ by fitting the present-day silicate-to-carbon dust mass ratio of the model to its observed value of 0.6 , inferred from observations of the infrared emission from the Diffuse Infrared Background Experiment (Dwek et al. 1997). 
The olivine fraction $f_{\mathrm{ol}}$ is chosen to reproduce the observed $\mathrm{Mg} / \mathrm{Si}$ ratio in dust using the simple relation for a given olivinepyroxene mixture:

$f_{\mathrm{ol}}=\frac{A_{\mathrm{Mg}}}{x A_{\mathrm{Si}}}-1$

Here $A_{\mathrm{Mg}}$ and $A_{\mathrm{Si}}$ are observed abundances for the elements $\mathrm{Si}$ and $\mathrm{Mg}$ bound in dust (in particles per million hydrogen atoms, ppm). The ratio $A_{\mathrm{Mg}} / A_{\mathrm{Si}}$ equals 1.06 or 1.07 for dust in the diffuse ISM, as given by Dwek (2005) or Whittet (2003), respectively, which results in a value of $f_{\mathrm{ol}}=0.32$. Although the $A_{\mathrm{Mg}} / A_{\mathrm{Si}}$ ratio obviously varies in different ISM phases, we use average dust abundances from the diffuse medium, since this constitutes a significant fraction of the total ISM mass, and only very little is known about the very cold dust in molecular clouds. Test calculations for different $A_{\mathrm{Mg}} / A_{\mathrm{Si}}$ ratios available from diffuse ISM studies showed no strong influence on dust masses, resulting in $4 \%$ change of total dust mass with $10 \%$ decrease of $A_{\mathrm{Mg}} / A_{\mathrm{Si}}$ ratio.

For given silicate composition, the growth species used to calculate the growth timescale, Eq. (30), is determined by the abundance of the least abundant species available for dust growth. This is either $\mathrm{Si}$ or $\mathrm{Mg}$, and we choose in Eq. (30)

$\epsilon=\left\{\begin{array}{cl}\frac{\Sigma_{\mathrm{Mg}}}{24 \Sigma_{\mathrm{H}}} & \text { for } \frac{\epsilon_{\mathrm{Mg}}}{v_{\mathrm{Mg}, c}}<\frac{\epsilon_{\mathrm{Si}}}{v_{\mathrm{Si}, c}} \\ \frac{\Sigma_{\mathrm{Si}}}{28 \Sigma_{\mathrm{H}}} & \text { for } \frac{\epsilon_{\mathrm{Mg}}}{v_{\mathrm{Mg}, c}}<\frac{\epsilon_{\mathrm{Si}}}{v_{\mathrm{Si}, c}}\end{array}\right.$,

where $v_{\mathrm{Si}, c}=1, v_{\mathrm{Mg}, c}=1.06$.

\subsubsection{Carbon dust}

The formula unit is the $\mathrm{C}$ atom, i.e., one has $v_{j, \mathrm{c}}=1$. It is assumed that $C$ is present in the gas phase in molecular clouds predominantly as free atoms or in a number of molecules bearing one $\mathrm{C}$ atom only and that these serve as growth species. Some fraction $f_{\mathrm{CO}}$ of the carbon is blocked in the $\mathrm{CO}$ molecule and is not available for carbon growth. The precise fraction cannot be fixed without calculating models for the chemistry of the molecular clouds. Observations indicate a $\mathrm{CO}$ abundance in molecular clouds of $20 \% \ldots 40 \%$ of the C abundance (e.g. Irvine et al. 1987; van Dishoek et al. 1993; van Dishoek \& Blake 1998). In the calculation we consider the two cases $\xi_{\mathrm{CO}}=0.2$ and $\xi_{\mathrm{CO}}=0.4$. The carbon abundance $\epsilon$ in Eqs. (30) and (34) is calculated as

$\epsilon=\left(1-\xi_{\mathrm{CO}}\right) \frac{\Sigma_{\mathrm{C}}-\Sigma_{\mathrm{C}, \mathrm{sic}}}{12 \Sigma_{\mathrm{H}}}$,

where $\Sigma_{\mathrm{C} \text {,sic }}$ is the surface density of $\mathrm{C}$ bound in silicon carbide dust

$\Sigma_{\mathrm{C}, \mathrm{sic}}=\frac{12}{40} \Sigma_{\text {sic }}$

\subsubsection{Iron dust}

The formula unit is the Fe atom, i.e., one has $v_{j, \mathrm{c}}=1$. It is assumed that $\mathrm{Fe}$ is present in the gas phase as free atoms, which are the growth species in this case. The iron abundance $\epsilon$ in Eqs. (30) and (34) is calculated as

$\epsilon=\frac{\Sigma_{\mathrm{Fe}}-\Sigma_{\mathrm{Fe}, \mathrm{sil}}}{56 \Sigma_{\mathrm{H}}}$

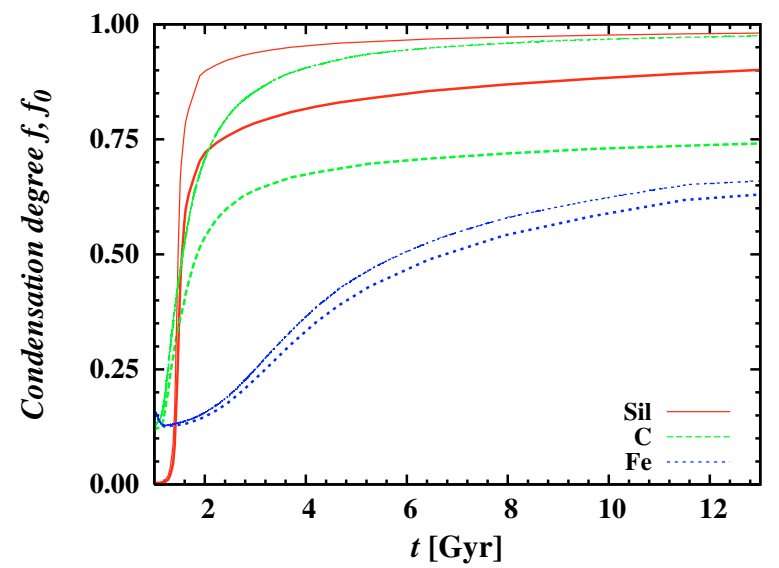

Fig. 14. Growth of dust in molecular clouds at the solar circle. Thick lines show $f$, the average degree of condensation of the key elements into dust for the dust species shown at the instant when the molecular clouds are dispersed and their material is mixed with the other phases of the ISM. Thin lines show $f_{0}$, the corresponding degree of condensation at the formation time of clouds. One always has $f_{0}<f$ since dust grains grow in molecular clouds and are partially destroyed again in the ISM outside of clouds until they enter the next cloud. Growth of iron dust in clouds starts with a significant time delay because of delayed iron production by SN Ia events. The calculation is for $\xi_{\mathrm{CO}}=0.2$; the result for $\xi_{\mathrm{CO}}=0.4$ is not shown because the corresponding curves are almost the sames.

where $\Sigma_{\mathrm{Fe} \text {,sil }}$ is the surface density of Fe bound in silicate dust species.

\section{Results of the evolution model for the solar circle}

\subsection{Evolution of MC-grown dust}

The model for the dust evolution considers silicate dust, carbon dust, and iron dust as species that grow in dense molecular clouds. The corresponding growth timescales $\tau_{\text {gr }}$ calculated from our Milky Way model at the solar circle are shown in Fig. 12.

During the first Gyr of evolution of the galactic disk, the metallicity at the solar circle is low $([\mathrm{Fe} / \mathrm{H}] \lessgtr-2$, cf. Fig. 3$)$ and the characteristic growth timescale of dust in clouds exceeds the average lifetime of dense molecular clouds of about $10 \mathrm{Myr}$ assumed in our model. Only small amounts of dust are added to the dust content of the interstellar matter during its cycling through clouds. This can be seen in Fig. 14, which shows the evolution of the initial value $f_{j, 0}$ of the degree of condensation of the key elements into dust, defined by Eq. (46), for each of the dust species $j$, and the average final degrees of condensation $f_{j}$, calculated according to Eq. (45) for the same species, if the clouds are finally dissolved. Both quantities, $f_{j, 0}$ and $f_{j}$, are calculated during the course of our model calculation for the evolution of the Milky Way at the solar circle. One always has $f_{0}<f$ since dust grains grow in molecular clouds and are partially destroyed again in the ISM outside of clouds until they enter the next cloud. Growth of iron dust in clouds starts with a significant time delay because of delayed iron production by SN Ia events.

During the first, about one Gyr the degree of condensation of refractory elements into dust increases only marginally by dust growth in molecular clouds. Therefore, the dust production in the Milky Way is almost completely determined by dust condensation in the ejecta of stars, and the dust content of the ISM is determined during this transient phase by dust injection from 


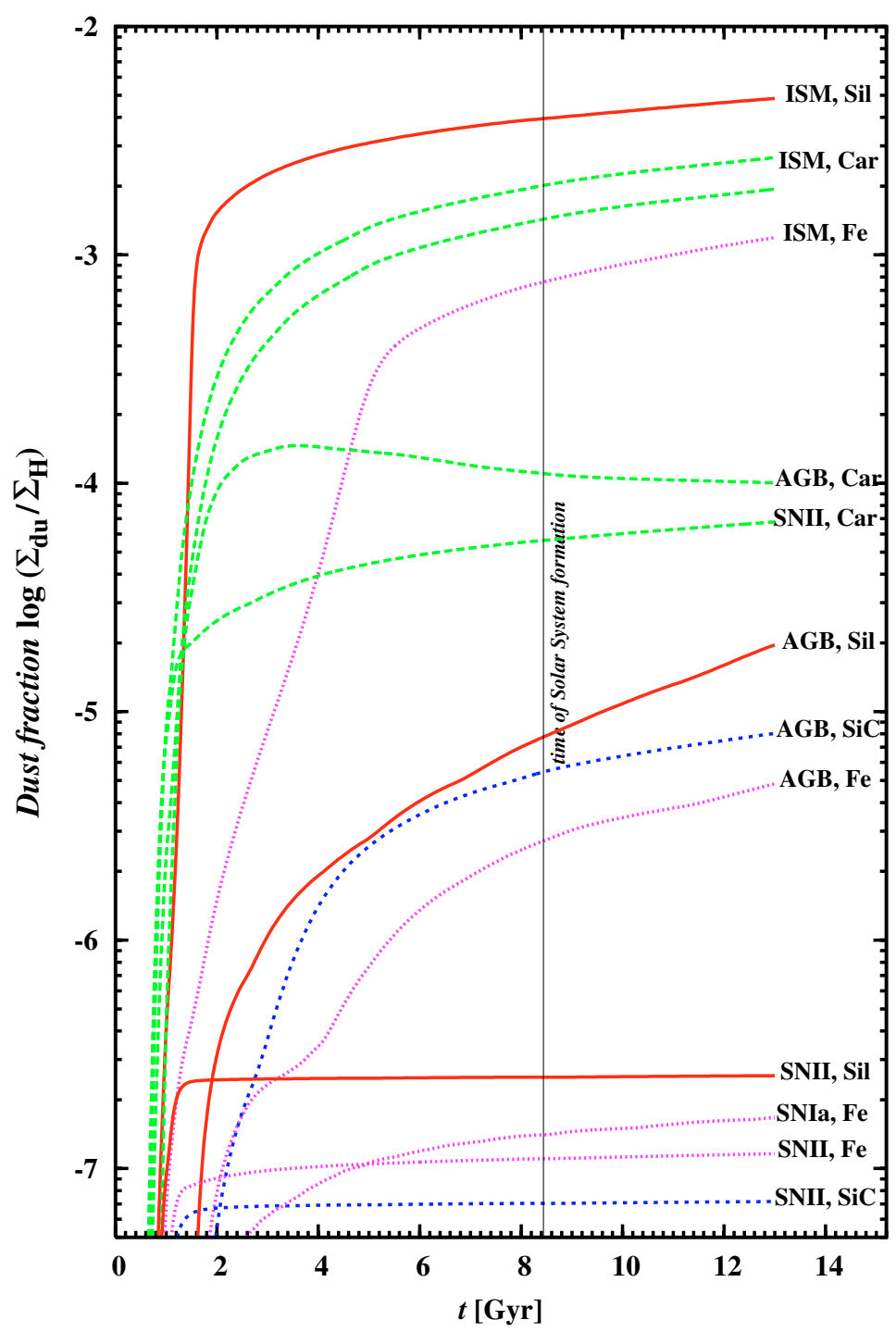

Fig. 15. Evolution of the dust mass fraction in the interstellar medium of the main interstellar dust components and of the stardust species at the solar circle. The dust grown in molecular clouds dominates the total dust mass of the interstellar medium. For carbon dust two results are shown corresponding to an assumed fraction of 0.2 resp. 0.4 of the carbon in molecular clouds blocked in the $\mathrm{CO}$ molecule. stars into the ISM and by dust destruction in the warm phase of the interstellar medium. Obviously the development would be considerably different if one has a strong starburst at early times and metallicity already becomes high before the first AGB stars appear, but this seems not to have happened in the case of our Milky Way.

Once the metallicity of the ISM has grown to a level of about $[\mathrm{Fe} / \mathrm{H}]=-2$, some dust starts to condense during the lifetime of molecular clouds, and their dust content at the instant of their dissolution somewhat exceeds their initial dust content. From this point on molecular clouds start to contribute to dust production in the galaxy.

If the metallicity has climbed after more than $2 \mathrm{Gyr}$ to a level of about $[\mathrm{Fe} / \mathrm{H}]=-1$, the degrees of condensation into dust $f_{j}$ at cloud dispersal are much higher than the degrees of condensation into dust $f_{j, 0}$ at cloud formation; in fact, dust growth almost runs into completion during the lifetime of the clouds. During each cycle step of interstellar matter through clouds, the matter is laden with fresh dust and this dust is mixed into the general ISM at cloud dispersal. The dust content of the ISM then is determined essentially by the equilibrium between dust growth in clouds and dust destruction in the warm phase of the interstellar medium.

The degree of condensation $f$ of carbon into carbon dust does not approach unity (see Fig. 14), since it is assumed that
20 to $40 \%$ of the carbon in molecular clouds forms CO and then is no longer available for dust condensation.

The iron dust abundance evolves somewhat differently from that of the silicate and carbon dust. The main reason is that most of the Fe is produced in SN Ia explosions and these turn on rather late due to the long lifetime of their low mass precursor stars. We also assumed in our model that SN Ia explosions do not start until the metallicity of the precursor stars has risen to $[\mathrm{Fe} / \mathrm{H}] \gtrsim$ -1 (see Sect. 2.1.5). A second reason is that it is assumed in our model of dust growth that the silicates grown in clouds contain a certain fraction of iron and the small fraction of iron initially produced by supernovae is then almost completely consumed by the growth of silicates with some iron content. This will change somewhat if the iron content of the silicates is not fixed, as in our present calculation, but will be determined from growth kinetics.

\subsection{Evolution of dust abundances}

Figure 15 shows the evolution of the various dust components during the 13 Gyr of evolution of the galactic disk. The dust components with index "ISM" are the isotopically normal grains grown in the interstellar medium. Surviving grains from stellar sources are characterised by an index "AGB" or "SN" if they are from AGB-stars or from supernova ejecta, respectively. The dust 
condensed in stellar ejecta (AGB stars, $\mathrm{SNe}$ ) only has a small abundance in the ISM. The condensation efficiencies of dust in supernovae used for the model calculation are given in Table 5.

The results depend on the efficiency of dust production by stars, dust condensation in molecular clouds, and dust destruction rates in the interstellar medium. The dust production by low and intermediate mass stars on the AGB is determined from the table of Ferrarotti \& Gail (2006) and the dust destruction rate from Jones et al. (1996). They are probably not too far from reality. The dust production efficiencies of massive stars are unknown. One has, however, one piece of information: the abundance ratios of the presolar dust grains from AGB stars and SNe. We have varied the supernova dust production efficiencies $\eta$ in Eqs. (16) ... (19) until the observed abundance ratios for silicate, carbon, and $\mathrm{SiC}$ dust from AGB and SNe sources is reproduced. Details are described in Sect. 5.3, the resulting efficiencies are listed in Table 5. These efficiencies are very low, probably since they also account for a number of destruction effects that prevent dust formed in SNe from escaping into the general ISM.

The dust population of the ISM in this model is dominated by dust grown in molecular clouds except for the very earliest times, where stardust dominates. The model shows that presolar dust grains with their isotopic anomalies revealing the origin of these grains are always a minor component of the interstellar dust. Most of the dust in the ISM has collected nearly all of its material from the interstellar gas phase and is isotopically inconspicuous. If new stars are formed from the ISM containing such a dust mixture, the dust in their protoplanetary accretion disks contains only a tiny fraction of presolar dust grains with isotopic anomalies. This fits well with the recent findings obtained with the nano-SIMS investigations of interplanetary dust grains by Messenger et al. (2003), which show that nearly all of the silicate grains from cometary nuclei, which should be dominated by interstellar grains, are isotopically normal ${ }^{6}$.

The population of stardust grains is dominated by grains from AGB stars because of the low efficiency of SN dust production. In our model the AGB dust is dominated by carbon dust; silicate dust and $\mathrm{SiC}$ dust are much less abundant. In meteorites presolar carbon dust in the state of graphite is the least abundant of these three components (cf. Nguyen et al. 2007). The discrepancy is certainly due (i) to the different survival properties of different kinds of dust material in the Solar System and the parent bodies of the meteorites, and (ii) the methods of laboratory investigations applied for different dust grains. This frustrates presently any comparison between abundances of different presolar species predicted by the model and observed in meteorites.

One outstanding feature of the abundance evolution of presolar dust grains is the rather late appearance of silicate and $\mathrm{SiC}$ as compared to carbon grains. This reflects that AGB stars synthesise the carbon required for soot formation from $\mathrm{He}$ and do not have to rely on external sources of heavy elements. In contrast to this, the Si-bearing dust components cannot be formed until enough $\mathrm{Si}$ is synthesised in supernova explosions and returned to the ISM, from which subsequent stellar generations inherit the $\mathrm{Si}$ required for formation of $\mathrm{Si}$-bearing species. This needs some time and additionally the precursor stars of the main sources of Si-bearing dust, the AGB stars, are rather long-lived low-mass stars (cf. Fig. 10). Presolar silicate dust grains in the ISM where a rather new phenomenon at the instant of Solar System formation.

\footnotetext{
${ }^{6}$ It is a little puzzling that the STARDUST particles analysed so far mainly seem to be material from the Solar System (see Zolensky et al. 2006; McKeegan et al. 2006).
}

The low abundance of silicate stardust may also explain the lack of crystalline silicate dust in the ISM, though a lot of crystalline dust is injected in to the interstellar medium by outflows from AGB stars. Even if there were no amorphization processes with energetic electrons and ions (cf. Demyk et al. 2004; Jäger et al. 2003), crystalline silicate dust ( $\leq 20 \%$ of the silicate dust injected by AGB stars) would be too rare compared to amorphous ISM dust to be observable by its absorption features.

\subsection{Efficiency of supernova dust production}

The dust mass produced in the ejecta of supernovae is not known. Observations indicate that only small amounts of dust condense and that only part of all SNe form dust. With the kind of model for dust evolution in the ISM we have developed, one can try to estimate the efficiency of dust production by supernovae for some dust species. This can be done by comparing the abundance ratios of supernova dust and AGB dust resulting from the model calculation with real observed abundance ratios of presolar dust grains with SN and AGB origin in meteorites. Only silicate, carbon, and $\mathrm{SiC}$ dust are presently suited for this, because the required data for presolar dust grains are available only for these dust species. Iron dust has not yet been detected as presolar dust so far, and it is unclear whether it really exists.

Such a comparison depends on some assumptions. The first one is that the production rate of dust by AGB stars is known with significantly better accuracy than the dust production rate of supernovae. The second basic assumption of a comparison between these kinds of data is that the fraction of the dust destroyed between the instant of its incorporation into the just-forming Solar System and the instant of laboratory investigation of presolar dust grains does not depend on the kind of stellar sources where the dust has formed, but only on its chemical composition. One has to assume, in other words, that the basic properties of AGB and SN dust with the same composition,

$$
\begin{aligned}
& \text { - its size spectrum, } \\
& \text { - its resilience against oxidation, and } \\
& \text { - its resilience against treatment by strong acids, }
\end{aligned}
$$

are the same.

\subsubsection{Silicon carbide}

The observed abundance ratio of X-type $\mathrm{SiC}$ grains and "mainstream" SiC grains in the presolar dust population isolated from meteorites is close to 0.01 (Hoppe et al. 2000). Fitting the efficiency $\eta_{\text {sic,SN II }}$ in Eq. (18) such that the calculated abundance ratio for SiC from supernovae of type II and AGB stars agrees with the observed abundance ratio yields $\eta_{\mathrm{sic}, \mathrm{SN} \text { II }}=5 \times 10^{-4}$. This ratio seems surprisingly low, but the low abundance of X-type $\mathrm{SiC}$ grains already shows that the efficiency of $\mathrm{SiC}$ dust formation in supernovae is low. The efficiency $\eta_{\text {sic,SN II }}$ of $\mathrm{SiC}$ dust condensation in supernova determined in this way is used for the final model calculation and is the one given in Table 5.

The abundance ratio for the $\mathrm{SiC}$ grains refers to grain abundances observed after isolating the grains from the meteorite matrix by a rather brutal treatment with oxidising agencies and by strong acids (cf. Amari et al. 1994), but it has been argued by Amari et al. $(1994,1995 a)$ that at most a small fraction of the grain material is lost during this procedure. On the other hand, the size distribution of $\mathrm{SiC}$ grains in the Murchison meteorite found by Daulton et al. (2003) shows a lack of grains smaller than $0.5 \mu \mathrm{m}$ diameter, which dominate in circumstellar 
Table 6. SN presolar dust fractions and corresponding derived efficiencies of dust production.

\begin{tabular}{lcc}
\hline \hline Dust species & Fraction of SN grains & $\eta_{j}$ \\
\hline \multirow{3}{*}{ Silicates } & 0.01 & 0.00015 \\
& 0.03 & 0.00035 \\
& 0.05 & 0.0005 \\
\hline \multirow{2}{*}{ Carbon } & 0.1 & 0.04 \\
& 0.3 & 0.15 \\
$\mathrm{SiC}$ & 0.5 & 0.20 \\
\hline
\end{tabular}

dust shells (e.g. Jura 1997), i.e., the small grains are already lost in the ISM or in the Solar System. If there were severe systematic differences in the mass fraction of sub-micron sized grains in the size distributions of $\mathrm{SiC}$ grains of $\mathrm{SN}$ and AGB origin, the abundance ratio derived from isolated $\mathrm{SiC}$ grains would be severely misleading, but presently we have no better data.

\subsubsection{Silicate dust}

The number of silicate grains from stellar sources detected in meteorites and interplanetary dust particles has been small up to now (Nguyen et al. 2007; Messenger et al. 2005). Besides the about some 100 silicate grains with isotopic anomalies attributable to an origin from AGB stars only a single grain has been detected with isotopic characteristics pointing unambiguously to an SN origin (Messenger et al. 2005). A few more have been detected that are also of likely SN origin (cf. Vollmer et al. 2007). The small numbers do not allow pinning down the abundance ratio of silicate dust from the two possible sources with any reliability, but we assume an abundance ratio of $3 \%$ as a working hypothesis. Then we can determine an efficiency of silicate dust formation in supernovae of $\eta_{\text {sil,SN II }}=3.5 \times 10^{-4}$. This is the value given in Table 5. The efficiencies for a somewhat lower and higher abundance ratio are shown for comparison in Table 6.

In contrast to the case of $\mathrm{SiC}$, the silicate grains are detected by scanning techniques from material that has not been prepared by chemical treatment. There is therefore no problem to be expected in the sense that part of the grain population is already destroyed by preparation methods before the particles are investigated.

\subsubsection{Carbon dust}

The abundance of presolar graphites from supernovae is highly uncertain. Chemically separated graphite fractions were further subdivided into low-density separates KE1 and high-density separates KFA1, KFB1, and KFC1 (Amari et al. 1994). While many - though not all - high-density graphites seem to have an AGB star origin (Croat et al. 2005), low-density graphites are ascribed to supernovae (Hoppe et al. 1995; Amari et al. 1995a; Travaglio et al. 1999), particularly inferred from isotope data of the low-density fraction KE3 (Amari et al. 1995b), which is the coarse-grained ( $>2 \mu \mathrm{m}$ ) subgroup making up $70 \%$ of KE1.

If all low-density graphites are from supernovae, this would correspond to a relative abundance of $67 \%$ (by weight). However, there is significant uncertainty which fractions of the various density separates do indeed correspond to a specific supernova or AGB star signature, so we adopt an abundance of $50 \pm 30 \%$ here (Hoppe, pers. comm.) and calculate 3 different cases for $10 \%, 30 \%$, and $50 \%$ of all graphites coming from supernovae. For the model results shown in the figures, we assumed a mass-fraction of $30 \%$. From this, one derives an efficiency of carbon dust formation in supernovae of $\eta_{\text {car,SN II }}=$ 0.15 . This is the value given in Table 5. This efficiency is much higher than in the two preceding cases and would mean that $\mathrm{SN}$ are mainly sources of carbon dust. Efficiencies for a lower (10\%) and higher (50\%) abundance ratio are shown in Table 6 for comparison.

Presolar graphite grains mainly have size $\gtrsim 1 \mu \mathrm{m}$ (e.g. Zinner 1997), while for carbon dust grains in circumstellar dust shells around AGB stars, one knows that they have sizes $\lessgtr 0.1 \mu \mathrm{m}$. Only a small fraction of grains from a large-size tail of the size distribution are found in the separates investigated in the laboratory. If the graphite grains formed in SN ejecta had systematically bigger sizes than those formed in AGB-star outflows (there is, however, no indication for this), the supernova graphite dust fraction found in the separates would overestimate the true abundance of graphite grains from supernovae, and our estimated efficiency $\eta_{\text {car,SN II }}$ would be too high.

The high condensation efficiency of carbon dust compared to that of SiC and silicate dust found in this model calculation seems likely since condensation of carbon dust only requires that carbon atoms in the carbon layer have to condense into dust particles, and no complicated mixing processes of the supernova ejecta between layers with different elemental composition are required, as for the dust species $\mathrm{SiC}$ and silicates. The formation of $\mathrm{SiC}$ requires that silicon from the layer containing the ashes of $\mathrm{O}$ burning and carbon from the layer containing the ashes of He burning are coming into contact without being completely mixed with the material from the thick $\mathrm{O}$ shell in between. This kind of incomplete mixing in a turbulent supernova shell is rather unlikely and therefore should only happen for a small fraction of the material.

\subsubsection{Need for dust accretion in the ISM}

The low efficiency of dust production by supernovae indicated by the rather low abundance of stardust of SN origin compared to stardust from AGB stars means that the supernovae cannot contribute substantially to dust in the ISM, contrary to what is frequently assumed. Therefore it is unavoidable that most of the dust mass observed in the ISM is formed in the ISM itself and not in stars. This has consequences for the dust production in young galaxies with low metallicity, where only supernovae can be sources of stardust. The high dust abundances observed in some high-redshift galaxies cannot, according to our results, result from the first generation of $\mathrm{SNe}$, but already requires additional accretion processes of heavy elements in interstellar clouds. This kind of dust evolution in young starburst galaxies will be treated in a separate paper.

\subsection{Dust composition}

Figure 16 shows our model results for the abundance evolution of the main dust-forming elements. Since we assumed a fixed composition of ISM silicates, the ratio between $\mathrm{Mg}, \mathrm{Si}$, and $\mathrm{O}$ does not change during evolution, but this is not the case for Fe, which is consumed both by silicate and iron dust-production. In the figure two lines are shown for carbon, corresponding to two different assumed fractions $\xi_{\mathrm{CO}}$ of the carbon in molecular clouds tied up in the in-reactive $\mathrm{CO}$ molecule. The upper one corresponds to $\xi_{\mathrm{CO}}=0.2$, the lower one to $\xi_{\mathrm{CO}}=0.4$, bracketing typically observed values (e.g. van Dishoek \& Blake 1998). 


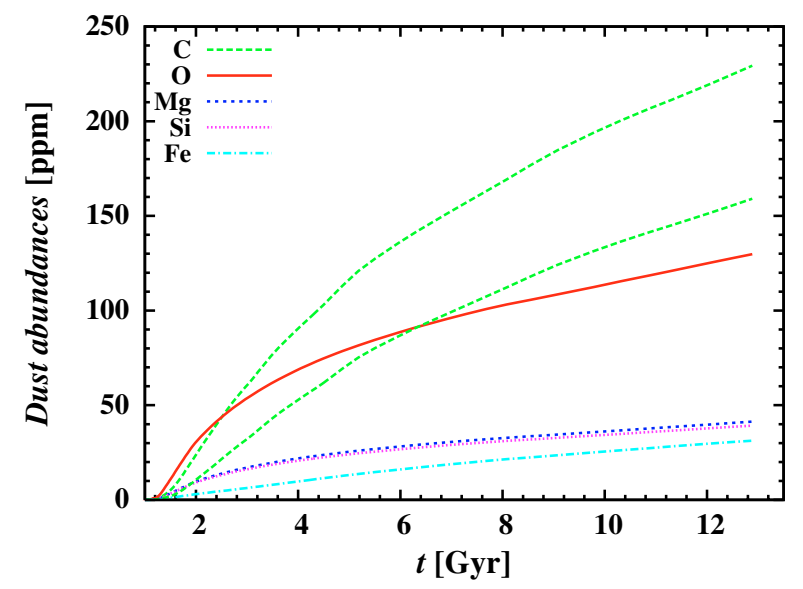

Fig. 16. Evolution of abundances in dust per million hydrogen atoms of the main dust-forming elements as predicted by the model calculation. Two lines are shown for carbon. The upper one is for the case that a fraction of $\xi_{\mathrm{CO}}=0.2$ of the carbon is bound in the in-reactive molecule $\mathrm{CO}$, the lower one for the case $\xi_{\mathrm{CO}}=0.4$.

A higher value of $\xi_{\mathrm{CO}}$ means that less carbon is available for dust formation.

The predicted dust abundances seems to be consistent with the composition of the local interstellar dust (Kimura et al. 2003b; Frisch 2006; Zubko et al. 2004). One should make such comparison with caution, since reference abundances may differ from those used in the present paper. Frisch (2006) derives the dust composition using the gas-phase abundances from the radiative transfer models of the local interstellar clouds (LIC) that are constrained by observations of ISM both inside and outside of the heliosphere. Our results are quite similar for $\mathrm{O}, \mathrm{Si}$, $\mathrm{Mg}, \mathrm{Fe}$, except $\mathrm{C}$ in carbon dust, which is missing from the LIC, possibly because it does not survive the acceleration mechanism Frisch (2006). At the same time, gas absorption measurements in lines of sight through the LIC and in situ dust measurements in Kimura et al. (2003b) indicate the same dust composition of local interstellar clouds as in warm diffuse clouds. In particular our results agree for carbon, iron, and oxygen, and are only about $10 \mathrm{ppm}$ higher for $\mathrm{Mg}, \mathrm{Si}$, which is within the accuracy of this kind of models.

The composition of the interstellar dust in the local ISM is also studied in Zubko et al. (2004) by simultaneous fitting of the interstellar extinction, diffuse IR emission, and abundance constraints. They considered different classes of models composed of silicates, graphite, PAHs, amorphous carbon, and composite particles. The main conclusion was that there is no unique dust model that fits the basic set of observational constraints, since several classes of models give equally good fits. Although a model with composite grains provides a better fit to the extinction and IR emission than a bare-grain model, the probing of interstellar dust models through small angle X-ray scattering favours models with bare silicates and graphite over those with composite particles (Dwek et al. 2004; Smith et al. 2006).

The observed gas-phase abundances of elements in diffuse interstellar clouds indicates various degrees of depletions of many of the dust-forming elements relative to their solar abundances. This is explained as resulting from their condensation in interstellar dust. The amount of the dust-forming elements locked up in interstellar dust (shown in Fig. 16), however, cannot reliably be derived from observations. The standard procedure is to instead determine gas-phase abundances of the elements and subtract these from some kind of "standard" cosmic element

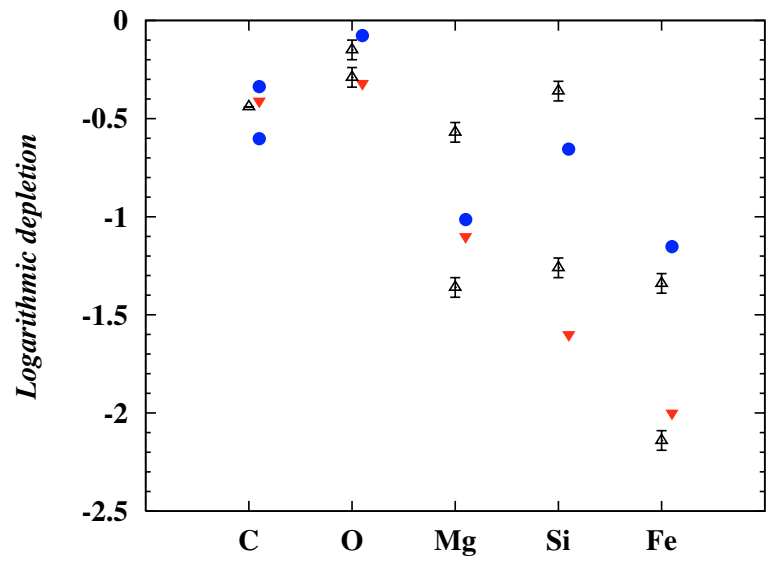

Fig. 17. Predicted average depletions of main dust-forming elements at the present time are shown with filled circles. Upper and lower points for depletions for carbon calculated with $\mathrm{CO}$ mass fraction 0.4 and 0.2 correspondingly. Upper and lower open triangles with error bars represent observed depletions in warm and cold diffuse clouds, respectively, from Welty et al. (1999) for C, Si, Fe and Cartledge et al. (2006) for O and $\mathrm{Mg}$. Filled triangles mark the average depletions in diffuse clouds (see Whittet 2003, and references therein).

abundances in order to determine how much of each element is condensed into interstellar dust (cf. Sembach \& Savage 1996, for a review). However, to draw conclusions about the dust composition from observed depletion patterns, one needs to make a decision about what set of abundances is used as the reference abundances for the elements. Frequently Solar System abundances, or abundances of nearby F \& G stars or of B stars, are adopted (cf. Tables 2 and 3), resulting in different dust compositions.

A modelling of the chemical evolution of the Galaxy including dust allows study of the evolution of the depletion of the gas abundances by dust condensation and a comparison of the model with presently observed data, since gas and dust abundances are known from calculations. However, a one-phase ISM model reflects properties of the dust averaged over the different ISM phases, so only a qualitative comparison of depletions is possible. One should notice that observed depletions are restricted to diffuse clouds, while molecular clouds are too opaque to be studied in absorption lines.

Our predicted averaged depletions, at the present time and at the solar circle in the ISM, for the 5 main dust-forming elements under consideration (C, O, Mg, Si, and Fe) are shown in Fig. 17. For comparison, observed depletions in warm and cold diffuse clouds from Welty et al. (1999) and Cartledge et al. (2006), and average depletions in diffuse clouds (see Whittet 2003) are also shown in the figure. The model calculation reasonably reproduces the observed values, except for a somewhat low calculated degree of depletion of Fe.

The degree of iron depletion cannot be increased by assigning a much longer destruction timescale $\tau_{j, \text { SNR }}$ for iron dust. A model calculation shows that this does not significantly increase the depletion because the lifetime of dust grains is limited in any case by the timescale of dust consumption by star formation, which is about $2.3 \mathrm{Gyr}$ and hence already not really long compared to the lifetime against destruction by shocks. Also a higher than assumed stability of Fe-bearing silicate does not help, since then a higher than observed depletion of $\mathrm{Si}$ is to be expected. The main reason for the low degree of depletion in the model seems to be that the fraction of $\mathrm{Fe}$ in the gas phase is not completely determined by the destruction of Fe-bearing grains by shocks in the warm phase but to a significant extent also by 


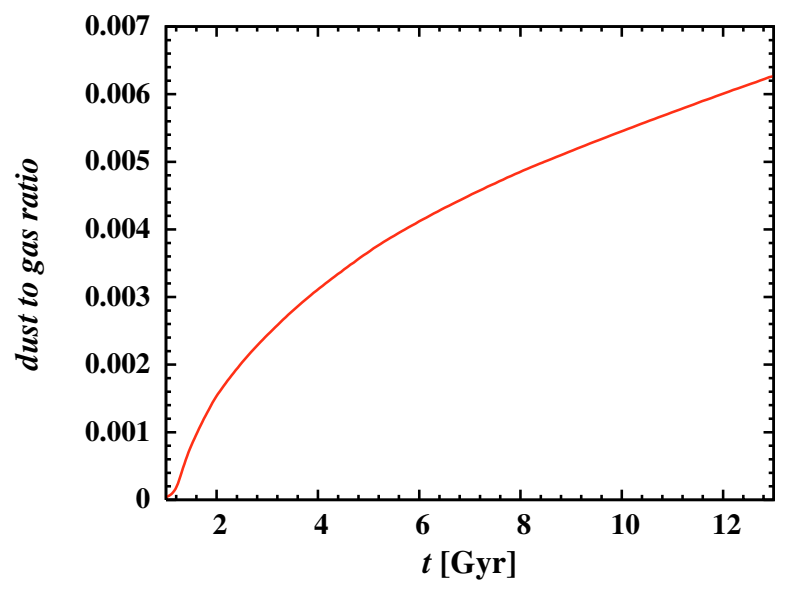

Fig. 18. Evolution of the dust-to-gas ratio at the solar circle as predicted by the model calculation.

return of gas-phase Fe by stars, which needs some time until it is cycled into clouds and depleted from the gas phase by dust growth processes. In our model, the degree of depletion of Fe (and this holds in principle for all refractory elements) is limited by the rather long time required for cycling of matter between the ISM matter not in dense clouds and the matter in dense clouds. The origin of the low degree of depletion of $\mathrm{Fe}$ in the model calculation is presently unclear, but probably bears physical significance and may indicate that, for iron, some slow accretion of $\mathrm{Fe}$ atoms from the gas phase into dust is also possible in the warm and/or cold phase of the ISM.

\subsection{Evolution of the dust to gas ratio}

Figure 18 shows the evolution of the dust-to-gas ratio according to the model calculation. The hydrogen gas-to-dust mass ratio is approximately 100 for the diffuse ISM averaged over long lines of sight passing through a number of interstellar clouds (Spitzer 1954). Recent studies of the hydrogen gas-to-dust ratio in the local interstellar cloud (Kimura et al. 2003a) also confirms the canonical value from Spitzer (1954). If one converts this to a ratio of dust mass to total gas mass, one gets a value of 0.007 . The value of the dust-to-gas ratio in our model for the present time ISM is close to the average value derived from observations. Our model therefore nicely reproduces the average dust mass fraction of the Milky Way in the solar vicinity.

\subsection{Dust input into the Solar System}

Figure 19 shows the composition of the interstellar dust mixture at the instant of Solar System formation and the present-day composition. Numerical values are given in Table 7. Both mixtures are not significantly different since the abundances of refractory elements in the ISM have changed only slightly over the past 4.56 Gyr (cf. Fig. 5). This dust mixture is clearly dominated by MC-grown dust and contains only a small fraction of stardust. The stardust is dominated by dust grains from AGB stars, meaning dust grains with SN origin form only a minor component.

The dust mixture at time of Solar System formation is the one from which the solid bodies in our planetary system formed. Relics of this dust mixture can be found in the Solar System in two types of objects: matrix material of primitive meteorites and in comets. However, until Solar System bodies formed from the dust component of the matter collapsed from some part of the
Table 7. Surface densities of different dust species at instant of Solar System formation and at the present time as predicted by the model calculation.

\begin{tabular}{llcc}
\hline \hline Dust species & Source & $\Sigma_{j, \mathrm{~d}}\left(t_{\mathrm{SSF}}\right)\left[M_{\odot} \mathrm{pc}^{-2}\right]$ & $\Sigma_{j, \mathrm{~d}}\left(t_{\mathrm{G}}\right)\left[M_{\odot} \mathrm{pc}^{-2}\right]$ \\
\hline \multirow{3}{*}{ Silicates } & AGB & $5.39 \times 10^{-5}$ & $1.12 \times 10^{-4}$ \\
& SN II & $2.51 \times 10^{-6}$ & $2.08 \times 10^{-6}$ \\
& ISM & $2.76 \times 10^{-2}$ & $2.76 \times 10^{-2}$ \\
\hline \multirow{3}{*}{ Carbon } & AGB & $7.73 \times 10^{-4}$ & $5.73 \times 10^{-4}$ \\
& SN II & $7.86 \times 10^{-5}$ & $7.73 \times 10^{-5}$ \\
& ISM & $1.43 \times 10^{-2}$ & $1.54 \times 10^{-2}$ \\
\hline \multirow{4}{*}{ Iron } & AGB & $1.89 \times 10^{-5}$ & $2.75 \times 10^{-5}$ \\
& SN II & $7.72 \times 10^{-7}$ & $6.63 \times 10^{-7}$ \\
& SN Ia & $9.83 \times 10^{-7}$ & $9.55 \times 10^{-7}$ \\
& ISM & $5.31 \times 10^{-3}$ & $6.78 \times 10^{-3}$ \\
\hline \multirow{2}{*}{ SiC } & AGB & $3.80 \times 10^{-5}$ & $4.58 \times 10^{-5}$ \\
& SN II & $8.21 \times 10^{-7}$ & $6.82 \times 10^{-7}$ \\
\hline \multirow{2}{*}{} & & &
\end{tabular}

parent molecular cloud into the protoplanetary accretion disk, the material underwent a number of alteration processes. Even the most primitive material in Solar System bodies is not simply unmodified ISM matter. For this reason meteoritic matrix material is presently not suited to a comparison with the model results, since the alteration processes on the parent bodies are presently not completely understood (cf. McSween et al. 2002). Material from comets is probably more suited; and once more detailed results from the STARDUST mission are available, it may be possible to compare the model predictions for the ISM dust composition entering the protoplanetary accretion disk of the Solar System with observations. Presently most of the analysed particles returned by the STARDUST mission seem to be material from the solar system (Zolensky et al. 2006; McKeegan et al. 2006).

Today one can only state that the dust mixture inherited by the Solar System from its parent molecular cloud and the current ISM dust mixture in the solar neighbourhood predicted by our model calculation are roughly in accord with the dust composition estimated by Pollack et al. (1994) from observations of the extinction properties of the dust material and considerations of element abundances, which is presently held to be the best estimate of the composition of the dust material from which the Solar System formed.

\section{Concluding remarks}

In the present paper we have developed a model for calculating the chemical evolution of of the Milky Way disk and the dust content of the interstellar medium in a consistent fashion, partially following the methods proposed by Tielens (1998) and in particular by Dwek (1998).

The chemical evolution part of the model for the Milky Ways disk mostly follows well-established methods. The model was checked with the standard tests applied to such models and reasonably reproduces the observational constraints. A new aspect is the use of the new tables of Nomoto et al. (2006) for the heavy element production by massive stars. This gives better agreement between the calculated evolution of the element abundances of the main dust-forming elements $(\mathrm{O}, \mathrm{Mg}, \mathrm{Si}, \mathrm{Fe})$ and the observed evolution of abundances in the ISM as witnessed by the variation in the atmospheric element abundances of main sequence $\mathrm{G}$ stars with metallicity. In particular the problem with the low $\mathrm{Mg}$ abundances disappears with the new results of Nomoto et al. (2006). A good reproduction of the abundance variations of the 

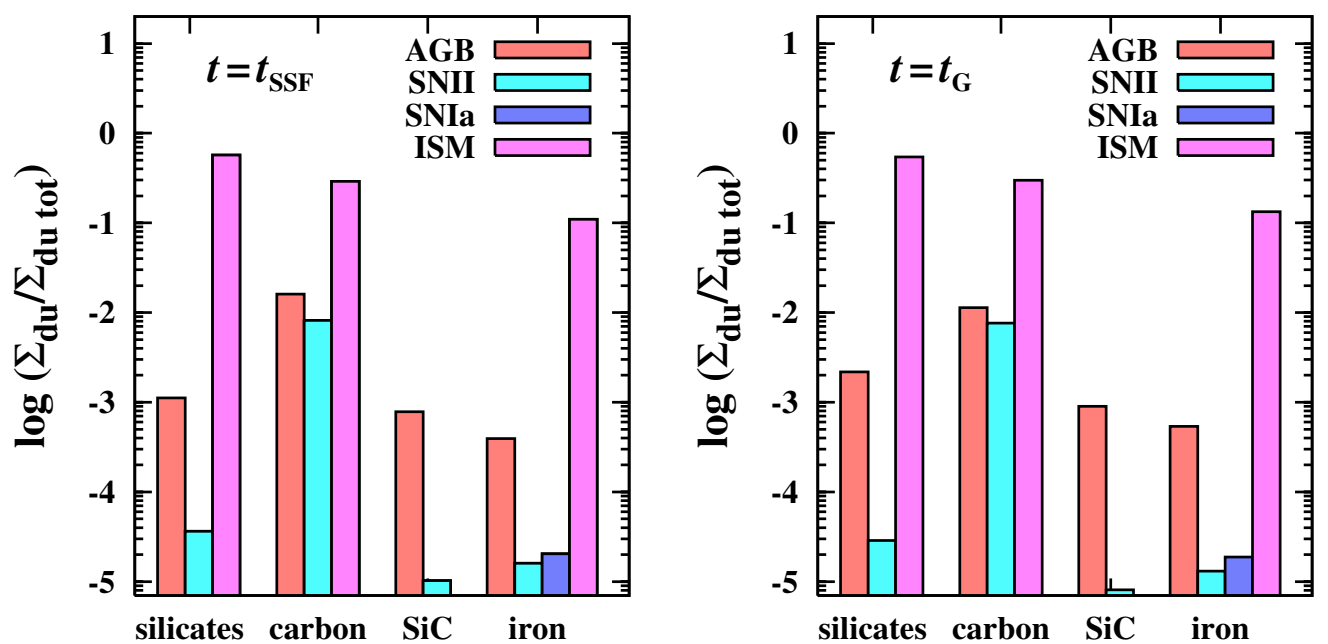

Fig. 19. Composition of the interstellar mixture of dust species grown in molecular clouds, the MC-grown dust, and of the presolar dust species from AGB stars and supernovae, the stardust, at the solar circle. Left: at the instant of Solar System formation. Right: for the present solar neighbourhood.

dust-forming elements is important if one tries to model the interstellar dust mixture.

The model for the evolution of the interstellar dust is based on three essential elements:

First, the model uses, for the dust input to the ISM by low and intermediate mass stars, the results of the model calculations of Ferrarotti \& Gail (2006), which combine synthetic AGB evolution models with models for circumstellar dust shells that include dust formation in the stellar wind. These models consistently describe for the first time the dependence of dust production by AGB stars on stellar initial mass and metallicity.

Second, the dust production by supernovae is described by a simple parametrisation, which has already been applied by Dwek (1998). A lot of information has accumulated over the years from the efforts of the meteoritic science community on studying nucleosynthetic processes in stars by analysing isotopic abundances in presolar dust grains. As a by-product, this provides us with abundance ratios of presolar dust grains from supernovae and from AGB stars. This allows the first estimates of the efficiency of dust production by supernovae by fitting calculated abundance ratios of stardust in the Solar System to observed abundance ratios of presolar dust grains from AGB stars and SNe. Applying these gauged efficiencies yields the unexpected result that dust production by massive stars is not important in the evolution of the ISM dust component, at least not for Pop II and Pop I metallicities.

Third, a simple approach is developed to include dust growth in molecular clouds into a model for the evolution of the interstellar dust. This approach is incomplete, in a sense, since the growth of dust (as its destruction) depends heavily on the phase structure of the interstellar gas (hot, warm, cold), which cannot be treated adequately within the approach generally used in chemical evolution models. So some quantities in the dust growth model, like the mass fraction of ISM matter in clouds, have to be taken from observations, but we only have data for these quantities for the current Milky Way. Since the distribution of the ISM over the phases is neither spatially nor temporal constant, the phase structure in a realistic modelling should be part of the model calculation. Nevertheless the present approach allows the first study of the evolution of the interstellar dust population, including several stardust species.
The results obtained with this model are in reasonable accord with, for instance, the observations of interstellar depletions of refractory elements. Again, one has the problem that the degree of depletion depends on the phases of the ISM, which are not adequately considered in the present model. At present we have started work on implementing our dust model in a chemodynamical evolution code (Berczik et al. 2003) that removes the shortcomings of the present model.

Acknowledgements. We acknowledge P. Hoppe for some very valuable discussions and comments on an earlier version of this paper. This work was supported by the Deutsche Forschungsgemeinschaft (DFG), Sonderforschungsbereich 439 "Galaxies in the Young Universe". S.Z. is supported in part also by the International Max-Planck Research School (IMPRS) Heidelberg

\section{References}

Allende Prieto, C., Lambert, D. L., \& Asplund, M. 2001, ApJ, 556, L63 Allende Prieto, C., Lambert, D. L., \& Asplund, M. 2002, ApJ, 573, L137

Amari, S., Lewis, R. S., \& Anders, E. 1994, Geochim. Cosmochim. Acta, 58, 459

Amari, S., Lewis, R. S., \& Anders, E. 1995a, Geochim. Cosmochim. Acta, 59, 1411

Amari, S., Zinner E., \& Anders, E. 1995b, ApJ, 447, L147

Anders, E., \& Grevesse, N. 1989, Geochim. Cosmochim. Acta, 53, 197

Asplund, M., Grevesse, N., \& Sauval, A. J. 2005, in Cosmic Abundances as Records of Stellar Evolution and Nucleosynthesis, ed. T. G. Barnes III, \& F. N. Bash (San Francisco: ASP), ASP Conf. Ser., 336, 25

Akerman, C. J., Carigi, L., Nissen, P. E., Pettini, M., \& Asplund, M. 2004 A\&A, 414,931

Alibes, A., Labay, J., \& Canal, R. 2001, A\&A, 370, 1103, 1121

Alongi, M., Bertelli, G., Bressan, A., et al. 1993, A\&AS, 97, 851

Asplund, M., Nordlund, Å, Trampedach, R., Allende Prieto, C., \& Stein, R. F. 2000, A\&A, 359, 729

Basu, S., Chaplin, W. J., Elsworth, Y., et al. 2007, ApJ, 655, 660

Bensby, T., \& Feltzing, S. 2006, MNRAS, 367, 1181

Bensby, T., Feltzing, S., Lundström, I., \& Ilyin, I. 2005, A\&A, 433, 185

Berczik, P., Hensler, G., Theis, C., \& Spurzem, R. 2003, Ap\&SS, 284, 865

Bernatowicz, T. J., \& Zinner, E. 1997, Astrophysical Implications of the

Laboratory Study of Presolar Materials (Woodbury: American Institute of Physics)

Bianchi, S., \& Schneider, R. 2007, MNRAS, 378, 973

Blitz, L., Fukui, Y., Kawamura, A., et al. 2007, in Prostars and Planets V, ed. B.

Reipurth, D. Jewitt, \& K. Keil (Tucson: University of Arizon Press), 81

Borkowski, K. J., Williams, B. J., Reynolds, S. P., et al. 2006, ApJ, 642, L141

Braun, R., \& Thilker, D. A. 2004, A\&A, 417, 421 
Bertelli, G., Bressan, A., Chiosi, C., Fagotto, F., \& Nasi, E. 1993, A\&AS, 106, 275

Bradley, J. 2003, in Astromineralogy, ed. Th. Henning, Lecture Notes in Physics, 609, 217

Bradley, J. P., Keller, L. P., Snow, T. P. et al. 1999, Science, 285, 1716

Bressan, A., Fagotto, F., Bertelli, G., \& Chiosi, C. 1993, A\&AS, 100, 647

Caffau, E., Bonifacio, P., Faraggiana, R., et al. 2005, A\&A, 441, 533

Cartledge, I. B., Lauroesch, J. T., Meyer, D. M., \& Sofia, U. J. 2006, ApJ, 641, 327

Cayrel, R., Depagne, E., Spite, M., et al. 2004, A\&A, 416, 117

Charbonnel, C., Meynet, G., Maeder, A., Schaller, G., \& Schaerer, D. 1993, A\&AS, 101, 415

Chen, Y. Q., Nissen P. E., Zhao, G., Zhang, H. W., \& Benoni, T. 2000, A\&AS, 141,491

Chiappini, C., Matteucci, F., \& Gratton, R. 1997, ApJ, 477, 765

Chiar, J. E., \& Tielens, A. G. G.M. 2006, ApJ, 637, 774

Chiosi, C. 1980, A\&A, 83, 206

Croat, T. K., Stadermann, F. J., \& Bernatowicz, T. J. 2005, ApJ, 631, 976

Cunha, K., \& Lambert, D. L. 1994, ApJ, 426, 170

Cunha, K., Hubeny, I., \& Lanz, T. 2006, ApJ, 647, L143

Daflon, S., \& Cunha, K. 2004, ApJ, 617, 1115

da Silva, L., Girardy, L., Pasquini, L., et al. 2006, A\&A, 458, 609

Daulton, T. L., Bernatowicz, T. J., Lewis, R. S., et al. 2003, Geochim. Cosmochim. Acta, 67, 4743

Delahaye, F., \& Pinsonneault, M. H. 2006, ApJ, 649, 529

Demyk, K., Jones, A. P., Dartois, E., et al. 1999, A\&A, 349, 267

Demyk, K., d'Hendecourt, L., Keroux, H., Jones, A. P., \& Borg, J. 2004, A\&A, 420, 547

Dickey, J. M. 1993, in The Minnesota Lectures on the Structure and Dynamics of the Milky Way, ed. R. M. Humphreys (San Francisco: ASP), ASP Conf. Ser., 39, 93

Dwarkadas, V. V. 2006, [arXiv: astro-ph/0612665]

Dwek, E. 1998, ApJ, 501, 643

Dwek, E. 2005, in The Spectral Energy Distributions of Gas-Rich Galaxies: Confronting Models with Data, ed. C. C. Popescu, \& R. J. Tuffs (Melville: American Institute of Physics), AIP Conf. Proc., 761, 103

Dwek, E., Arendt, R. G., Fixsen, D. J., et al. 1997, ApJ, 475, 565

Dwek, E., Zubko, V., Arendt, R., \& Smith, R. 2004, in Astrophysics of Dust, ed. A. Witt, ASP Conf. Ser., 309, 499

Dwek, E., Galliano, F., \& Jones, A. P. 2007, ApJ, 662, 927

Dopita, M. A., \& Ryder, S. D. 1994, ApJ, 430, 163

Draine, B. 1990, in The Evolution of the Insterstellar Medium, ed. L. Blitz (San Francisco: ASP), ASP Conf. Proc., 12, 193

Edmunds, M. G. 2001, MNRAS, 328, 223

Edvardsson, B., Andersen, J., Gustafsson, B., et al. 1993, A\&A, 275, 101

Elmegreen, B. G., \& Scalo, J. 2006, ApJ, 636, 149

Ercolano, B., Barlow, M. J., \& Sugerman, B. E. K. 2007, MNRAS, 375, 753

Falgarone, E., Pineau Des Forêts, G., Hily-Blant, P., \& Schilke, P. 2006, A\&A, 452,511

Feldman, U., \& Widing, K. G. 2003, Space Sci. Rev., 107, 665

Ferrarotti, A. S., \& Gail, H.-P. 2006, A\&A, 447, 553

François, P., Matteucci, F., Cayrel, R., et al. 2004, A\&A, 421, 613

Frisch, P. C. 2006, [arXiv: astro-ph/0603745]

Gratton, R. G., \& Sneden, C. 1991, A\&A, 241, 501

Grevesse, N., \& Sauval, A. J. 1998, Space Sci. Rev., 85, 161

Gil-Pons, P., \& García-Berro, E. 2002, A\&A, 396, 589

Gilmore, G., Wyse, R., \& Kuijen, K. 1989, in Evolutionary Phenomena in Galaxies, ed. J. Beckman, \& B. Pagel (Cambridge: Cambridge University Press), 172

Goswami, A., \& Prantzos, N. 2000, A\&A, 359, 191

Hachisu, I., Kato, M., \& Nomoto, K. 1996, ApJ, 470, L97

Hachisu, I., Kato, M., \& Nomoto K. 1999, ApJ, 522, 487

Helmi, A., Navarro, J. F., Nordström, B., et al. 2006, MNRAS, 365, 1309

Hirashita, H. 2000, ApJ, 531, 693

Hollenbach, D., \& Salpeter, E. E. 1979, J. Chem. Phys., 53, 79

Holmberg, J., \& Flynn, C. 2004, MNRAS, 352, 440

Holmgren, D. E., Brown, P. J. F., Dufton, P. L., \& Keenan, F. P. 1990, ApJ, 364, 657

Holweger, H. 2001, in Solar and Galactic Composition, ed. R. F. Wimmer-Schweingruber (Melville: American Institute of Physics), AIP Conf. Proc., 598, 23

Hoppe, P. 2004, in Astrophysics of Dust, ed. A. N. Witt, G. C. Clayton, \& B. T. Draine (San Francisco: ASP), ASP Conf. Ser., 309, 265

Hoppe, P., Amari, S., Zinner, E., \& Lewis, R. S. 1995, Geochim. Cosmochim. Acta, 59, 4029

Hoppe, P., Strebel, R., Eberhardt, P., Amari, S., \& Lewis, R. S. 2000, Meteoritics and Planetary Science, 35,1157

Inoue, A. K. 2003, PASJ, 55, 901
Irvine, W. M., Goldsmith, P. F., \& Hjalmarson, ̊. 1987, in Interstellar Processes, ed. D. J. Hollenbach, H. A. Thronson (Dordrecht: Reidel), 561

Iwamoto, K., Brachwitz, F., Nomoto, K., et al. 1999, ApJS, 125, 439

Jäger, C., Fabian, D., Schrempel, F., et al. 2003, A\&A, 401, 57

Jenkins, E. B. 2004, in Origin and Evolution of the Elements, ed. A. Mc William,

\& M. Rauch (Cambridge: Cambridge University Press), 336

Jenniskens, P., Baratta, G. A., Kouchi, A., et al. 1993, A\&A, 273, 583

Jones, A. P. 1990, MNRAS, 245, 331

Jones, A. P. 2000, JGR, 105, 10257

Jones, A. P. 2005, in The dusty and molecular universe: a prelude to Herschel and ALMA, ed. A. Wilson (Noordwijk: ESA), ESA SP-577, 239

Jones, A. P., Tielens, A. G. G. M., Hollenbach, D. J., \& McKee, C. F. 1994, ApJ, 433, 797

Jones A. P., Tielens, A. G. G. M., \& Hollenbach, D. J. 1996, ApJ, 469, 740

Jonsell, K., Edvardsson, B., Gustafsson, B., et al. 2005, A\&A, 440, 321

Jura, M. 1997, in Astrophysical Implications of the Laboratory Study of Presolar Materials, ed. T. J. Bernatowicz, \& E. Zinner (Woodbury: American Institute of Physics), AIP Conf. Proc., 402, 379

Karakas, A. I., \& Lattanzio, J. C. 2003, Publications of the Astronomical Society of Australia, 20, 279

Karakas, A. I., Lugaro, M., Ugalde, C., Wiescher, M., \& Görres, J. 2006, New Astron. Rev., 50, 500

Kemper, F., de Koter, A., Waters, L. F. B. M., Bouwman, J., \& Tielens, A. G. G. M. 2002, A\&A, 384, 585

Kemper, F., Vriend, W. J., \& Tielens, A. G. G. M. 2004, ApJ, 609, 826

Kennicutt, R. C. 1998, ApJ, 498, 541

Kimura, H., Mann, I., \& Jessberger, E. K. 2003a, ApJ, 582, 846

Kimura, H., Mann, I., \& Jessberger, E. K. 2003b, ApJ, 583, 314

Kobayashi, C., Umeda, H., Nomoto, K., Tominaga, N., \& Ohkubo, T. 2006, ApJ, 653,1145

Kozasa, T., Hasegawa, H., \& Nomoto, K. 1989 ApJ, 344, 325

Kroupa, P. 2002, Science, 295, 82

Kroupa, P., Tout, C. A., \& Gilmore, G. 1993, MNRAS, 262, 545

Krumholz, M. R., Matzner, C. D., \& McKee, C. F. 2006, ApJ, 653, 361

Lamers, H. J. G. L. M., Nota, A., Panagia, N., Smith, L. J., \& Langer, N. 2001, ApJ, 551, 764

Leisawitz, D., Bash, F. N., \& Thaddeus, P. 1989, ApJS, 70, 731

Liffman, K., \& Clayton, D. D. 1989, ApJ, 340, 853

Lisenfeld, U., \& Ferrara, A. 1998, ApJ, 496, 145

Lyubimkov, L. S., Rostopchin, S. I., \& Lambert, D. L. 2004, MNRAS, 351, 745

Lyubimkov, L. S., Rostopchin, S. I., Rachkovskaya, T. M., Poklad, D. B., \& Lambert, D. L. 2005, MNRAS, 358, 193

Marchenko, S. V., \& Moffat, A. F. J. 2006 [arXiv:astro-ph/0610531]

Mathis, J. S., Rumpl, W., \& Nordsieck, K. H. 1977, ApJ, 217, 425

Matteucci, F. 2003, The chemical evolution of the galaxy, (Kluwer Academic Publishers)

Matteucci, F., \& Greggio, L. 1986, A\&A, 154, 279

Matzner, C. D. 2002, ApJ, 566, 302

McKeegan, K. D., Aléon, J., Bradley, J., et al. 2006, Science, 314, 1724

McSween, H. Y., Ghosh, A., Grimm, R. E., Wilson, L., \& Young, E. D. 2002, in Asteroids III, ed. W. F. Bottke et al. (Tucson: University of Arizona Press), 559

Melendez, J., \& Barbuy, B. 2002, ApJ, 575, 474

Mera, D., Chabrier, G., \& Schaeffer, R. 1998, A\&A, 330, 937

Messenger, S., Keller, L. P., Stadermann, F. J., Walker, R. M., \& Zinner, E. 2003, Science, 300, 105

Messenger, S., Keller, L. P., \& Lauretta, D. S. 2005, Science, 309, 737

Meynet, G., \& Maeder, A. 2005, A\&A, 429, 581

Miller, G. E., \& Scalo, J. M. 1979, ApJS, 41, 513

Min, M., Waters, L. B. F. M., de Koter, A., et al. 2007, A\&A, 462, 667

Molster, F. J., \& Waters, L. B. F. M. 2003, in Astromineralogy, ed. Th. Henning, Lecture Notes in Physics, 609, 121

Morgan, H. L., \& Edmunds, M. G. 2003, MNRAS, 343, 427

Nittler, L. R., \& Alexander, C. M. O’D. 2003, Geochim. Cosmochim. Acta, 24, 4961

Nguyen, A. N., Stadermann, F. J., Zinner, E., et al. 2007, ApJ, 656, 1223

Nozawa, T., Kozasa, T., Umeda, H., Maeda, K., \& Nomoto, K. 2003, ApJ, 598, 785

Nomoto, K., Nozomu, T., Umeda, H., Kobayashi, C., \& Maeda, K. 2006, Nuclear Physics A, 777, 424

Nordström, B., Andersen, J., Holmberg, J., et al. 2004, PASA, 21, 129

Nozawa, T., Kozasa, T., Habe, A., et al. 2007 [arXiv: 0706. 0383]

Olling, R. P., \& Merrifield, M. R. 2001, MNRAS, 326, 164

Pagel, B. 1997, Nucleosynthesis and Galactic Chemical Evolution (Cambridge: Cambridge University Press)

Palme, H., \& Jones, A. 2003, in Treatise on Geochemistry, ed. H. D. Holland, \& K. K. Turekian (Amsterdam: Elsevier), 41

Pollack, J. B., Hollenbach, D. J., Beckwith, S., et al. 1994, ApJ, 421, 615 
Pont, F, \& Eyer, L. 2004, MNRAS, 351, 487

Portinari, L., Chiosi, C., \& Bressan, A. 1998, A\&A, 334, 505

Preibisch, T., Brown, A. G. A., Bridges, T., Guenther, E., \& Zinnecker, H. 2002, AJ, 124, 404

Ramírez, I., Allende Prieto, C., \& Lambert, D. L. 2007, A\&A, 465, 271

Rana, N. 1991, ARA\&A, 29, 129

Reddy, B. E., Tomkin, J., Lambert, D. L., \& Allende Prieto, C. 2003, MNRAS, 340,304

Reiteri, C. M., Villata, M., \& Navarro, J. F. 1996, A\&A, 305, 105

Reddy, B. E., Lambert, D. L., \& Allende Prieto, C. 2006, MNRAS, 367, 1329

Rocha-Pinto, H. J., \& Maciel, W. J. 1996, MNRAS, 279, 447

Rocha-Pinto, H. J., Maciel, W. J., Scalo, J., \& Flynn, C. 2000, A\&A, 358, 850

Rolleston, W. R. J., Smartt, S. J., Dufton, P. L., \& Ryans, R. S. I. 2000, A\&A, 363, 537

Salpeter, E. E. 1955 ApJ, 121, 161

Savage, B. D., \& Sembach, K. R. 1996, ARA\&A,34, 279

Scalo, J. 2005, in The Initial Mass Function 50 Years Later, ed. E. Corbelli, \& F. Palle (Dordrecht: Springer), Astrophys. Sp. Sci. Library, 327, 23

Scalo, J., \& Elmegreen, B. G. 2004, ARA\&A, 42, 275

Schaller, G., Schaerer, D., Meynet, G., \& Maeder, A. 1992, A\&AS, 96, 269

Schaerer, D., Meynet, G., Maeder, A., \& Schaller, G. 1993, A\&AS, 98, 523

Schneider, R., Ferrara, A., \& Salvaterra, R. 2004, MNRAS, 351, 1379

Shull, J. M., \& Draine, B. T. 1987, in Interstellar Processes, ed. D. J. Hollenbach, \& H. A. Thronson (Dordrecht: Reidel), 283

Smith, N. 2006, [arXiv: astro-ph/0609422]

Smith, N., \& Owocki, S. P. 2006, ApJ, 645, L45

Smith, R. K., Dame, T. M., Costantini, E., \& Predehl, P. 2006, ApJ, 648, 452

Sofia, U. J., \& Meyer, D. M. 2001, ApJ, 554, L221

Soubiran, C., \& Girard, P. 2005 A\&A, 438, 139

Spitzer, L., Jr. 1954, ApJ, 120, 1

Sugerman, B. E. K., Ercolano, B., Barlow, M., et al. 2006, Science, 313, 196

Talbot, R. J., \& Arnett, W. D. 1975, ApJ, 197, 551

Tammann, G., Löffler, W., \& Schröder, A. 1994, ApJS, 92, 487

Tielens, A. G. G. M. 1998, ApJ, 499, 267

Tielens, A. G. G. M. 2003, Science, 300, 68

Tielens, A. G. G. M. 2005, The physics and chemistry of the interstellar medium (Cambridge: Cambridge University Press)
Tielens, A. G. G. M. Waters, L. B. F. M., \& Bernatowicz, T. J. 2005, in Chondrites and the Protoplanetary Disk, ed. A. N. Krot, E. R. D. Scott, \& B. Reipurth (San Francisco: ASP), ASP Conf., 341, 605

Timmes, F. X., Woosley, S. E., \& Weaver, T. A. 1995, ApJS, 98, 617

Todini, P., \& Ferrara, A. 2001, MNRAS, 325, 736

Travaglio, C., Gallino, R., Amari, S., et al. 1999, ApJ, 510, 325

van den Hoek, L. B., \& Groenewegen, M. A. T. 1997, A\&AS, 123, 305

van der Hucht, K. A. 2003, in A Massive Star Odyssey: From Main Sequence to

Supernova, IAU Symp., 212, 441

van Dishoeck, E. F., \& Blake, G. A. 1998, ARA\&A, 36, 317

van Dishoeck, E. F., Blake, G. A., Draine, B. T., \& Lunine, J. I. 2003, in

Protostars and Planets III, ed. E. H. Levy, \& J. I. Lunine (Tucson: University of Arizona Press), 163

van Loon, J. T., Groenewegen, M. A. T., de Koter, A., et al. 1999, A\&A, 351, 559

Vollmer, C., Hoppe, P., Brenker, F. E., \& Holzapfel, C. 2007, Lunar and Planetary Institute Conference Abstracts, 38, 1262

Venn, K. A., Irwin, M., Shetrone, M. D., et al. 2004, ApJ, 128, 1177

Vorobyov, E. I., \& Shchekinov, Y. A. 2006, New Astron., 11, 240

Watson, W. D. 1975, in Atomic and Molecular Physics of the Interstellar Matter, Les Houches 1974, ed. R. Balian, P. Encrenaz, \& J. Lequex (Amsterdam: North-Holland Publ. Comp.), 177

Welty, D. E., Hobbs, L. M., Lauroesch, J. T., et al. 1999, ApJS, 124, 465

Whittet, D. C. B. 2003, Dust in the galactic environment (Institute of Physics Publishing)

Williams, J. P., \& McKee, C. F. 1997, ApJ, 476, 166

Woitke, P., Dominik, C., \& Sedlmayer, E. 1993, A\&A, 274, 451

Woosley, S. E., \& Weaver, T. A. 1995, ApJS, 101, 181

Whelan, J., \& Iben Jr., I. 1973, ApJ, 186, 1007

Zinner, E. 1998, in Astrophysical Implications of the Laboratory Study of Presolar Materials, ed. T. J. Bernatowicz, \& E. Zinner (Woodbury: American Institute of Physics), AIP Conf. Proc., 402, 3

Zinner, E. 1998, Ann. Rev. Earth \& Plan. Sci., 26, 147

Zolensky, M. E., Zega, T. J., Yano, H., et al. 2006, Science, 314, 1735

Zubko, V., Dwek, E., \& Arendt, R. G. 2004, ApJS, 152, 211 


\section{Appendix A: Dust formation by AGB stars}

Table A.1 gives dust masses returned by low and intermediate mass stars separately for all dust species considered in Ferrarotti \& Gail (2006). The calculations are done as in that paper, but a finer grid of metallicities and initial masses are used. The initial models at the begin of thermal pulsing not in the model set of the Geneva group are determind by linear interpolation between the avalable models. 
S. Zhukovska et al.: Evolution of interstellar dust and stardust, Online Material p 2

Table A.1. Ejected mass of dust by AGB stars for different chemistries of M-stars, S-stars and C-stars for different initial masses $M_{*}$ and metallicities $Z$. All masses are given in Solar mass.

\begin{tabular}{|c|c|c|c|c|c|c|c|c|c|c|c|}
\hline \multirow[b]{2}{*}{$M_{*}$} & \multirow[b]{2}{*}{ forsterite } & \multicolumn{4}{|c|}{ M stars } & \multirow[b]{2}{*}{ iron } & \multicolumn{2}{|c|}{ S stars } & \multicolumn{3}{|c|}{ C stars } \\
\hline & & fayalite & enstatite & ferrosilite & quartz & & quartz & iron & $\mathrm{SiC}$ & carbon & iron \\
\hline \multicolumn{12}{|c|}{$Z=0.001$} \\
\hline 1.00 & 0.0 & 0.0 & 0.0 & 0.0 & 0.0 & 0.0 & 0.0 & 0.0 & 0.0 & 0.0 & 0.0 \\
\hline 1.10 & 0.0 & 0.0 & 0.0 & 0.0 & 0.0 & 0.0 & 0.0 & 0.0 & 0.0 & $4.124 \times 10^{-6}$ & 0.0 \\
\hline 1.20 & 0.0 & 0.0 & 0.0 & 0.0 & 0.0 & 0.0 & 0.0 & 0.0 & 0.0 & $3.585 \times 10^{-4}$ & 0.0 \\
\hline 1.25 & 0.0 & 0.0 & 0.0 & 0.0 & 0.0 & 0.0 & 0.0 & 0.0 & 0.0 & $5.976 \times 10^{-4}$ & 0.0 \\
\hline 1.30 & 0.0 & 0.0 & 0.0 & 0.0 & 0.0 & 0.0 & 0.0 & 0.0 & 0.0 & $8.878 \times 10^{-4}$ & 0.0 \\
\hline 1.40 & 0.0 & 0.0 & 0.0 & 0.0 & 0.0 & 0.0 & 0.0 & 0.0 & 0.0 & $1.298 \times 10^{-3}$ & 0.0 \\
\hline 1.50 & 0.0 & 0.0 & 0.0 & 0.0 & 0.0 & 0.0 & 0.0 & 0.0 & 0.0 & $1.985 \times 10^{-3}$ & 0.0 \\
\hline 1.60 & 0.0 & 0.0 & 0.0 & 0.0 & 0.0 & 0.0 & 0.0 & 0.0 & 0.0 & $2.520 \times 10^{-3}$ & 0.0 \\
\hline 1.70 & 0.0 & 0.0 & 0.0 & 0.0 & 0.0 & 0.0 & 0.0 & 0.0 & 0.0 & $3.159 \times 10^{-3}$ & 0.0 \\
\hline 1.80 & 0.0 & 0.0 & 0.0 & 0.0 & 0.0 & 0.0 & 0.0 & 0.0 & $1.122 \times 10^{-9}$ & $3.827 \times 10^{-3}$ & 0.0 \\
\hline 1.90 & 0.0 & 0.0 & 0.0 & 0.0 & 0.0 & 0.0 & 0.0 & 0.0 & $1.221 \times 10^{-9}$ & $4.209 \times 10^{-3}$ & 0.0 \\
\hline 2.00 & 0.0 & 0.0 & 0.0 & 0.0 & 0.0 & 0.0 & 0.0 & 0.0 & $1.367 \times 10^{-9}$ & $4.538 \times 10^{-3}$ & 0.0 \\
\hline 2.10 & 0.0 & 0.0 & 0.0 & 0.0 & 0.0 & 0.0 & 0.0 & 0.0 & $1.483 \times 10^{-9}$ & $4.799 \times 10^{-3}$ & 0.0 \\
\hline 2.20 & 0.0 & 0.0 & 0.0 & 0.0 & 0.0 & 0.0 & 0.0 & 0.0 & $1.629 \times 10^{-9}$ & $5.096 \times 10^{-3}$ & 0.0 \\
\hline 2.30 & 0.0 & 0.0 & 0.0 & 0.0 & 0.0 & 0.0 & 0.0 & 0.0 & $1.748 \times 10^{-9}$ & $5.416 \times 10^{-3}$ & 0.0 \\
\hline 2.40 & 0.0 & 0.0 & 0.0 & 0.0 & 0.0 & 0.0 & 0.0 & 0.0 & $1.895 \times 10^{-9}$ & $5.687 \times 10^{-3}$ & 0.0 \\
\hline 2.50 & 0.0 & 0.0 & 0.0 & 0.0 & 0.0 & 0.0 & 0.0 & 0.0 & $2.035 \times 10^{-9}$ & $5.788 \times 10^{-3}$ & 0.0 \\
\hline 3.00 & 0.0 & 0.0 & 0.0 & 0.0 & 0.0 & 0.0 & 0.0 & 0.0 & $3.006 \times 10^{-9}$ & $6.997 \times 10^{-3}$ & 0.0 \\
\hline 3.50 & 0.0 & 0.0 & 0.0 & 0.0 & 0.0 & 0.0 & 0.0 & 0.0 & $3.842 \times 10^{-9}$ & $9.751 \times 10^{-3}$ & 0.0 \\
\hline 4.00 & 0.0 & 0.0 & 0.0 & 0.0 & 0.0 & 0.0 & 0.0 & 0.0 & $4.802 \times 10^{-9}$ & $1.283 \times 10^{-2}$ & 0.0 \\
\hline 4.01 & 0.0 & 0.0 & 0.0 & 0.0 & 0.0 & 0.0 & 0.0 & 0.0 & $4.825 \times 10^{-9}$ & $1.286 \times 10^{-2}$ & 0.0 \\
\hline 4.50 & $1.318 \times 10^{-9}$ & 0.0 & 0.0 & 0.0 & 0.0 & $1.308 \times 10^{-9}$ & 0.0 & 0.0 & $2.185 \times 10^{-9}$ & $5.789 \times 10^{-4}$ & 0.0 \\
\hline 5.00 & $1.744 \times 10^{-9}$ & 0.0 & $1.252 \times 10^{-9}$ & 0.0 & 0.0 & $1.699 \times 10^{-9}$ & 0.0 & 0.0 & $2.407 \times 10^{-9}$ & $5.812 \times 10^{-4}$ & 0.0 \\
\hline 5.50 & $2.203 \times 10^{-9}$ & 0.0 & $1.557 \times 10^{-9}$ & 0.0 & 0.0 & $2.100 \times 10^{-9}$ & 0.0 & 0.0 & $2.454 \times 10^{-9}$ & $5.903 \times 10^{-4}$ & 0.0 \\
\hline 6.00 & $2.698 \times 10^{-9}$ & 0.0 & $1.875 \times 10^{-9}$ & 0.0 & 0.0 & $2.525 \times 10^{-9}$ & 0.0 & 0.0 & $2.537 \times 10^{-9}$ & $5.968 \times 10^{-4}$ & 0.0 \\
\hline 6.50 & $3.234 \times 10^{-9}$ & 0.0 & $2.215 \times 10^{-9}$ & 0.0 & 0.0 & $2.981 \times 10^{-9}$ & 0.0 & 0.0 & $2.743 \times 10^{-9}$ & $6.158 \times 10^{-4}$ & 0.0 \\
\hline 7.00 & $3.795 \times 10^{-9}$ & 0.0 & $2.574 \times 10^{-9}$ & 0.0 & 0.0 & $3.467 \times 10^{-9}$ & 0.0 & 0.0 & $2.904 \times 10^{-9}$ & $6.266 \times 10^{-4}$ & 0.0 \\
\hline \multicolumn{12}{|c|}{$Z=0.002$} \\
\hline 1.00 & 0.0 & 0.0 & 0.0 & 0.0 & 0.0 & 0.0 & 0.0 & 0.0 & 0.0 & 0.0 & 0.0 \\
\hline 1.10 & 0.0 & 0.0 & 0.0 & 0.0 & 0.0 & 0.0 & 0.0 & 0.0 & 0.0 & $3.101 \times 10^{-6}$ & 0.0 \\
\hline 1.20 & 0.0 & 0.0 & 0.0 & 0.0 & 0.0 & 0.0 & 0.0 & 0.0 & $1.275 \times 10^{-9}$ & $3.620 \times 10^{-4}$ & 0.0 \\
\hline 1.25 & 0.0 & 0.0 & 0.0 & 0.0 & 0.0 & 0.0 & 0.0 & 0.0 & $1.743 \times 10^{-9}$ & $4.663 \times 10^{-4}$ & 0.0 \\
\hline 1.30 & 0.0 & 0.0 & 0.0 & 0.0 & 0.0 & 0.0 & 0.0 & 0.0 & $2.822 \times 10^{-9}$ & $7.462 \times 10^{-4}$ & 0.0 \\
\hline 1.40 & 0.0 & 0.0 & 0.0 & 0.0 & 0.0 & 0.0 & 0.0 & 0.0 & $5.096 \times 10^{-9}$ & $1.217 \times 10^{-3}$ & 0.0 \\
\hline 1.50 & 0.0 & 0.0 & 0.0 & 0.0 & 0.0 & 0.0 & $2.527 \times 10^{-9}$ & 0.0 & $7.099 \times 10^{-9}$ & $1.735 \times 10^{-3}$ & 0.0 \\
\hline 1.60 & 0.0 & 0.0 & 0.0 & 0.0 & 0.0 & 0.0 & 0.0 & 0.0 & $8.149 \times 10^{-9}$ & $2.195 \times 10^{-3}$ & 0.0 \\
\hline 1.70 & 0.0 & 0.0 & 0.0 & 0.0 & 0.0 & 0.0 & 0.0 & 0.0 & $8.617 \times 10^{-9}$ & $2.632 \times 10^{-3}$ & 0.0 \\
\hline 1.80 & 0.0 & 0.0 & 0.0 & 0.0 & 0.0 & 0.0 & 0.0 & 0.0 & $1.013 \times 10^{-8}$ & $3.188 \times 10^{-3}$ & 0.0 \\
\hline 1.90 & 0.0 & 0.0 & 0.0 & 0.0 & 0.0 & 0.0 & 0.0 & 0.0 & $1.150 \times 10^{-8}$ & $3.674 \times 10^{-3}$ & 0.0 \\
\hline 2.00 & 0.0 & 0.0 & 0.0 & 0.0 & 0.0 & 0.0 & 0.0 & 0.0 & $1.318 \times 10^{-8}$ & $4.047 \times 10^{-3}$ & 0.0 \\
\hline 2.10 & 0.0 & 0.0 & 0.0 & 0.0 & 0.0 & 0.0 & 0.0 & 0.0 & $1.415 \times 10^{-8}$ & $4.156 \times 10^{-3}$ & 0.0 \\
\hline 2.20 & 0.0 & 0.0 & 0.0 & 0.0 & 0.0 & 0.0 & 0.0 & 0.0 & $1.559 \times 10^{-8}$ & $4.610 \times 10^{-3}$ & 0.0 \\
\hline 2.30 & 0.0 & 0.0 & 0.0 & 0.0 & 0.0 & 0.0 & 0.0 & 0.0 & $1.704 \times 10^{-8}$ & $4.674 \times 10^{-3}$ & 0.0 \\
\hline 2.40 & 0.0 & 0.0 & 0.0 & 0.0 & 0.0 & 0.0 & 0.0 & 0.0 & $1.843 \times 10^{-8}$ & $4.950 \times 10^{-3}$ & 0.0 \\
\hline 2.50 & 0.0 & 0.0 & 0.0 & 0.0 & 0.0 & 0.0 & 0.0 & 0.0 & $2.024 \times 10^{-8}$ & $5.097 \times 10^{-3}$ & 0.0 \\
\hline 3.00 & 0.0 & 0.0 & 0.0 & 0.0 & 0.0 & 0.0 & 0.0 & 0.0 & $3.049 \times 10^{-8}$ & $6.147 \times 10^{-3}$ & 0.0 \\
\hline 3.50 & 0.0 & 0.0 & 0.0 & 0.0 & 0.0 & 0.0 & 0.0 & 0.0 & $4.078 \times 10^{-8}$ & $8.874 \times 10^{-3}$ & 0.0 \\
\hline 4.00 & 0.0 & 0.0 & 0.0 & 0.0 & 0.0 & 0.0 & 0.0 & 0.0 & $5.399 \times 10^{-8}$ & $1.194 \times 10^{-2}$ & 0.0 \\
\hline 4.01 & 0.0 & 0.0 & 0.0 & 0.0 & 0.0 & 0.0 & 0.0 & 0.0 & $5.429 \times 10^{-8}$ & $1.200 \times 10^{-2}$ & 0.0 \\
\hline 4.50 & $7.918 \times 10^{-9}$ & $3.261 \times 10^{-9}$ & $5.418 \times 10^{-9}$ & $1.103 \times 10^{-9}$ & $1.110 \times 10^{-9}$ & $9.549 \times 10^{-9}$ & 0.0 & 0.0 & $2.000 \times 10^{-8}$ & $5.119 \times 10^{-4}$ & $1.931 \times 10^{-9}$ \\
\hline 5.00 & $1.047 \times 10^{-8}$ & $4.022 \times 10^{-9}$ & $7.056 \times 10^{-9}$ & $1.240 \times 10^{-9}$ & $1.413 \times 10^{-9}$ & $1.219 \times 10^{-8}$ & 0.0 & 0.0 & $2.269 \times 10^{-8}$ & $5.098 \times 10^{-4}$ & $2.732 \times 10^{-9}$ \\
\hline 5.50 & $1.336 \times 10^{-8}$ & $4.815 \times 10^{-9}$ & $8.881 \times 10^{-9}$ & $1.484 \times 10^{-9}$ & $1.759 \times 10^{-9}$ & $1.531 \times 10^{-8}$ & 0.0 & 0.0 & $2.399 \times 10^{-8}$ & $5.189 \times 10^{-4}$ & $2.658 \times 10^{-9}$ \\
\hline 6.00 & $1.640 \times 10^{-8}$ & $5.590 \times 10^{-9}$ & $1.072 \times 10^{-8}$ & $1.781 \times 10^{-9}$ & $2.114 \times 10^{-9}$ & $1.855 \times 10^{-8}$ & 0.0 & 0.0 & $2.527 \times 10^{-8}$ & $5.335 \times 10^{-4}$ & $2.275 \times 10^{-9}$ \\
\hline 6.50 & $1.992 \times 10^{-8}$ & $6.466 \times 10^{-9}$ & $1.281 \times 10^{-8}$ & $2.128 \times 10^{-9}$ & $2.519 \times 10^{-9}$ & $2.229 \times 10^{-8}$ & 0.0 & 0.0 & $2.768 \times 10^{-8}$ & $5.435 \times 10^{-4}$ & $2.537 \times 10^{-9}$ \\
\hline 7.00 & $2.373 \times 10^{-8}$ & $7.391 \times 10^{-9}$ & $1.507 \times 10^{-8}$ & $2.508 \times 10^{-9}$ & $2.952 \times 10^{-9}$ & $2.634 \times 10^{-8}$ & 0.0 & 0.0 & $3.052 \times 10^{-8}$ & $5.486 \times 10^{-4}$ & $3.025 \times 10^{-9}$ \\
\hline
\end{tabular}


Table A.1. continued.

\begin{tabular}{|c|c|c|c|c|c|c|c|c|c|c|c|}
\hline \multirow[b]{2}{*}{$M_{*}$} & \multicolumn{6}{|c|}{ M stars } & \multicolumn{3}{|c|}{ S stars } & \multicolumn{2}{|l|}{ C stars } \\
\hline & forsterite & fayalite & enstatite & ferrosilite & quartz & iron & quartz & iron & $\mathrm{SiC}$ & carbon & iron \\
\hline & & & & & & $Z=0.004$ & & & & & \\
\hline 1.00 & 0.0 & 0.0 & 0.0 & 0.0 & 0.0 & 0.0 & 0.0 & 0.0 & 0.0 & 0.0 & 0.0 \\
\hline 1.10 & 0.0 & 0.0 & 0.0 & 0.0 & 0.0 & 0.0 & 0.0 & 0.0 & 0.0 & $1.486 \times 10^{-6}$ & 0.0 \\
\hline 1.20 & 0.0 & 0.0 & 0.0 & 0.0 & 0.0 & 0.0 & 0.0 & 0.0 & $2.554 \times 10^{-8}$ & $2.259 \times 10^{-4}$ & 0.0 \\
\hline 1.25 & 0.0 & 0.0 & 0.0 & 0.0 & 0.0 & 0.0 & 0.0 & 0.0 & $7.297 \times 10^{-8}$ & $5.476 \times 10^{-4}$ & 0.0 \\
\hline 1.30 & 0.0 & 0.0 & 0.0 & 0.0 & 0.0 & 0.0 & 0.0 & 0.0 & $9.196 \times 10^{-8}$ & $7.373 \times 10^{-4}$ & 0.0 \\
\hline 1.40 & 0.0 & 0.0 & 0.0 & 0.0 & 0.0 & 0.0 & 0.0 & 0.0 & $1.226 \times 10^{-7}$ & $1.164 \times 10^{-3}$ & 0.0 \\
\hline 1.50 & 0.0 & 0.0 & 0.0 & 0.0 & 0.0 & 0.0 & 0.0 & 0.0 & $1.652 \times 10^{-7}$ & $1.700 \times 10^{-3}$ & 0.0 \\
\hline 1.60 & 0.0 & 0.0 & 0.0 & 0.0 & 0.0 & 0.0 & 0.0 & 0.0 & $1.949 \times 10^{-7}$ & $2.442 \times 10^{-3}$ & 0.0 \\
\hline 1.70 & 0.0 & 0.0 & 0.0 & 0.0 & 0.0 & 0.0 & 0.0 & 0.0 & $2.053 \times 10^{-7}$ & $3.175 \times 10^{-3}$ & 0.0 \\
\hline 1.80 & 0.0 & 0.0 & 0.0 & 0.0 & 0.0 & 0.0 & 0.0 & 0.0 & $2.349 \times 10^{-7}$ & $4.090 \times 10^{-3}$ & 0.0 \\
\hline 1.90 & 0.0 & 0.0 & 0.0 & 0.0 & 0.0 & 0.0 & 0.0 & 0.0 & $2.587 \times 10^{-7}$ & $5.151 \times 10^{-3}$ & 0.0 \\
\hline 2.00 & 0.0 & 0.0 & 0.0 & 0.0 & 0.0 & 0.0 & 0.0 & 0.0 & $2.830 \times 10^{-7}$ & $5.589 \times 10^{-3}$ & 0.0 \\
\hline 2.10 & 0.0 & 0.0 & 0.0 & 0.0 & 0.0 & 0.0 & 0.0 & 0.0 & $2.881 \times 10^{-7}$ & $6.268 \times 10^{-3}$ & 0.0 \\
\hline 2.20 & 0.0 & 0.0 & 0.0 & 0.0 & 0.0 & 0.0 & 0.0 & 0.0 & $3.391 \times 10^{-7}$ & $6.850 \times 10^{-3}$ & 0.0 \\
\hline 2.30 & 0.0 & 0.0 & 0.0 & 0.0 & 0.0 & 0.0 & 0.0 & 0.0 & $3.454 \times 10^{-7}$ & $7.532 \times 10^{-3}$ & 0.0 \\
\hline 2.40 & 0.0 & 0.0 & 0.0 & 0.0 & 0.0 & 0.0 & 0.0 & 0.0 & $3.793 \times 10^{-7}$ & $7.909 \times 10^{-3}$ & 0.0 \\
\hline 2.50 & 0.0 & 0.0 & 0.0 & 0.0 & 0.0 & 0.0 & $2.350 \times 10^{-8}$ & 0.0 & $3.990 \times 10^{-7}$ & $8.647 \times 10^{-3}$ & $\begin{array}{l}3 \\
3\end{array}$ \\
\hline 3.00 & 0.0 & 0.0 & 0.0 & 0.0 & 0.0 & 0.0 & 0.0 & 0.0 & $6.012 \times 10^{-7}$ & $1.429 \times 10^{-2}$ & $2.095 \times 10^{-9}$ \\
\hline 3.50 & 0.0 & 0.0 & 0.0 & 0.0 & 0.0 & 0.0 & 0.0 & 0.0 & $1.128 \times 10^{-6}$ & $8.011 \times 10^{-3}$ & $1.799 \times 10^{-9}$ \\
\hline 4.00 & 0.0 & 0.0 & 0.0 & 0.0 & 0.0 & 0.0 & 0.0 & 0.0 & $1.595 \times 10^{-6}$ & $1.063 \times 10^{-2}$ & $2.083 \times 10^{-9}$ \\
\hline 4.01 & 0.0 & 0.0 & 0.0 & 0.0 & 0.0 & 0.0 & 0.0 & 0.0 & $1.605 \times 10^{-6}$ & $1.068 \times 10^{-2}$ & $2.087 \times 10^{-9}$ \\
\hline 4.50 & $2.032 \times 10^{-7}$ & $1.530 \times 10^{-7}$ & $1.003 \times 10^{-7}$ & $4.704 \times 10^{-8}$ & $1.900 \times 10^{-8}$ & $82.632 \times 10^{-7}$ & 0.0 & 0.0 & $3.989 \times 10^{-7}$ & $4.100 \times 10^{-4}$ & ${ }^{4} 1.686 \times 10^{-8}$ \\
\hline 5.00 & $2.709 \times 10^{-7}$ & $1.972 \times 10^{-7}$ & $1.332 \times 10^{-7}$ & $5.893 \times 10^{-8}$ & $2.452 \times 10^{-8}$ & $83.292 \times 10^{-7}$ & 0.0 & 0.0 & $4.525 \times 10^{-7}$ & $4.018 \times 10^{-4}$ & ${ }^{4} 2.418 \times 10^{-8}$ \\
\hline 5.50 & $3.042 \times 10^{-7}$ & $2.151 \times 10^{-7}$ & $1.514 \times 10^{-7}$ & $6.360 \times 10^{-8}$ & $2.778 \times 10^{-8}$ & $83.682 \times 10^{-7}$ & 0.0 & $2.681 \times 10^{-9}$ & $5.197 \times 10^{-7}$ & $4.075 \times 10^{-4}$ & $3.039 \times 10^{-8}$ \\
\hline 6.00 & $3.462 \times 10^{-7}$ & $2.398 \times 10^{-7}$ & $1.742 \times 10^{-7}$ & $7.091 \times 10^{-8}$ & $3.200 \times 10^{-8}$ & $84.256 \times 10^{-7}$ & 0.0 & 0.0 & $5.530 \times 10^{-7}$ & $4.098 \times 10^{-4}$ & $2.775 \times 10^{-8}$ \\
\hline 6.50 & $3.944 \times 10^{-7}$ & $2.700 \times 10^{-7}$ & $1.983 \times 10^{-7}$ & $7.935 \times 10^{-8}$ & $3.657 \times 10^{-8}$ & $84.867 \times 10^{-7}$ & 0.0 & $1.574 \times 10^{-9}$ & $6.357 \times 10^{-7}$ & $4.219 \times 10^{-4}$ & $3.861 \times 10^{-8}$ \\
\hline 7.00 & $4.524 \times 10^{-7}$ & $3.073 \times 10^{-7}$ & $2.275 \times 10^{-7}$ & $9.031 \times 10^{-8}$ & $4.210 \times 10^{-8}$ & ${ }^{8} 5.641 \times 10^{-7}$ & 0.0 & $2.388 \times 10^{-9}$ & $6.819 \times 10^{-7}$ & $4.251 \times 10^{-4}$ & $4.691 \times 10^{-8}$ \\
\hline & & & & & & $Z=0.008$ & & & & & \\
\hline 1.00 & 0.0 & 0.0 & 0.0 & 0.0 & 0.0 & 0.0 & 0.0 & 0.0 & 0.0 & 0.0 & 0.0 \\
\hline 1.10 & $1.974 \times 10^{-7}$ & $1.538 \times 10^{-7}$ & $1.015 \times 10^{-7}$ & $4.305 \times 10^{-8}$ & $1.423 \times 10^{-8}$ & $8 \quad 1.896 \times 10^{-7}$ & 0.0 & 0.0 & $1.876 \times 10^{-8}$ & $2.870 \times 10^{-7}$ & $72.982 \times 10^{-8}$ \\
\hline 1.20 & $1.395 \times 10^{-6}$ & $1.085 \times 10^{-6}$ & $7.034 \times 10^{-7}$ & $3.095 \times 10^{-7}$ & $1.026 \times 10^{-7}$ & $71.440 \times 10^{-6}$ & 0.0 & 0.0 & $1.265 \times 10^{-6}$ & $1.433 \times 10^{-4}$ & ${ }^{4} 8.789 \times 10^{-7}$ \\
\hline 1.25 & $7.559 \times 10^{-7}$ & $6.018 \times 10^{-7}$ & $3.728 \times 10^{-7}$ & $1.721 \times 10^{-7}$ & $5.555 \times 10^{-8}$ & ${ }^{8} 6.965 \times 10^{-7}$ & 0.0 & $2.697 \times 10^{-8}$ & $4.326 \times 10^{-6}$ & $1.766 \times 10^{-4}$ & ${ }^{4} 1.651 \times 10^{-6}$ \\
\hline 1.30 & $7.729 \times 10^{-7}$ & $6.216 \times 10^{-7}$ & $3.838 \times 10^{-7}$ & $1.832 \times 10^{-7}$ & $5.735 \times 10^{-8}$ & $87.543 \times 10^{-7}$ & 0.0 & $2.628 \times 10^{-8}$ & $5.022 \times 10^{-6}$ & $4.135 \times 10^{-4}$ & $4.041 \times 10^{-8}$ \\
\hline 1.40 & $8.243 \times 10^{-7}$ & $6.744 \times 10^{-7}$ & $3.963 \times 10^{-7}$ & $2.028 \times 10^{-7}$ & $6.196 \times 10^{-8}$ & $88.762 \times 10^{-7}$ & 0.0 & 0.0 & $5.923 \times 10^{-6}$ & $6.370 \times 10^{-4}$ & $4.760 \times 10^{-8}$ \\
\hline 1.50 & 0.0 & 0.0 & 0.0 & 0.0 & 0.0 & 0.0 & 0.0 & 0.0 & $7.079 \times 10^{-6}$ & $8.459 \times 10^{-4}$ & $4.951 \times 10^{-8}$ \\
\hline 1.60 & 0.0 & 0.0 & 0.0 & 0.0 & 0.0 & 0.0 & 0.0 & 0.0 & $8.829 \times 10^{-6}$ & $1.425 \times 10^{-3}$ & $3.255 \times 10^{-8}$ \\
\hline 1.70 & 0.0 & 0.0 & 0.0 & 0.0 & 0.0 & 0.0 & 0.0 & 0.0 & $1.006 \times 10^{-5}$ & $2.027 \times 10^{-3}$ & $1.853 \times 10^{-8}$ \\
\hline 1.80 & 0.0 & 0.0 & 0.0 & 0.0 & 0.0 & 0.0 & 0.0 & 0.0 & $1.075 \times 10^{-5}$ & $2.680 \times 10^{-3}$ & $1.647 \times 10^{-8}$ \\
\hline 1.90 & 0.0 & 0.0 & 0.0 & 0.0 & 0.0 & 0.0 & 0.0 & 0.0 & $1.181 \times 10^{-5}$ & $3.515 \times 10^{-3}$ & $1.071 \times 10^{-8}$ \\
\hline 2.00 & 0.0 & 0.0 & 0.0 & 0.0 & 0.0 & 0.0 & 0.0 & 0.0 & $1.314 \times 10^{-5}$ & $4.414 \times 10^{-3}$ & $1.057 \times 10^{-8}$ \\
\hline 2.10 & 0.0 & 0.0 & 0.0 & 0.0 & 0.0 & 0.0 & 0.0 & 0.0 & $1.430 \times 10^{-5}$ & $5.319 \times 10^{-3}$ & $9.333 \times 10^{-9}$ \\
\hline 2.20 & 0.0 & 0.0 & 0.0 & 0.0 & 0.0 & 0.0 & 0.0 & 0.0 & $1.452 \times 10^{-5}$ & $5.941 \times 10^{-3}$ & $9.407 \times 10^{-9}$ \\
\hline 2.30 & 0.0 & 0.0 & 0.0 & 0.0 & 0.0 & 0.0 & 0.0 & 0.0 & $1.586 \times 10^{-5}$ & $6.763 \times 10^{-3}$ & $9.468 \times 10^{-9}$ \\
\hline 2.40 & 0.0 & 0.0 & 0.0 & 0.0 & 0.0 & 0.0 & 0.0 & 0.0 & $1.811 \times 10^{-5}$ & $7.417 \times 10^{-3}$ & $9.564 \times 10^{-9}$ \\
\hline 2.50 & 0.0 & 0.0 & 0.0 & 0.0 & 0.0 & 0.0 & 0.0 & 0.0 & $1.867 \times 10^{-5}$ & $8.352 \times 10^{-3}$ & $9.541 \times 10^{-9}$ \\
\hline 3.00 & 0.0 & 0.0 & 0.0 & 0.0 & 0.0 & 0.0 & 0.0 & 0.0 & $2.673 \times 10^{-5}$ & $1.434 \times 10^{-2}$ & $29.258 \times 10^{-9}$ \\
\hline 3.50 & 0.0 & 0.0 & 0.0 & 0.0 & 0.0 & 0.0 & 0.0 & 0.0 & $4.243 \times 10^{-5}$ & $6.406 \times 10^{-3}$ & $2.500 \times 10^{-8}$ \\
\hline 4.00 & 0.0 & 0.0 & 0.0 & 0.0 & 0.0 & 0.0 & 0.0 & 0.0 & $5.424 \times 10^{-5}$ & $8.072 \times 10^{-3}$ & $2.810 \times 10^{-8}$ \\
\hline 4.01 & 0.0 & 0.0 & 0.0 & 0.0 & 0.0 & 0.0 & 0.0 & 0.0 & $5.448 \times 10^{-5}$ & $8.130 \times 10^{-3}$ & $2.808 \times 10^{-8}$ \\
\hline 4.50 & $1.108 \times 10^{-4}$ & $8.666 \times 10^{-5}$ & $5.988 \times 10^{-5}$ & $2.551 \times 10^{-5}$ & $7.295 \times 10^{-6}$ & ${ }^{6} 6.689 \times 10^{-5}$ & 0.0 & $2.869 \times 10^{-7}$ & $6.921 \times 10^{-6}$ & $2.101 \times 10^{-4}$ & $5.099 \times 10^{-7}$ \\
\hline 5.00 & $1.285 \times 10^{-4}$ & $9.920 \times 10^{-5}$ & $7.088 \times 10^{-5}$ & $2.931 \times 10^{-5}$ & $8.319 \times 10^{-6}$ & $67.444 \times 10^{-5}$ & 50.0 & 0.0 & $9.286 \times 10^{-6}$ & $2.276 \times 10^{-4}$ & $42.649 \times 10^{-6}$ \\
\hline 5.50 & $1.486 \times 10^{-4}$ & $1.138 \times 10^{-4}$ & $8.282 \times 10^{-5}$ & $3.350 \times 10^{-5}$ & $9.388 \times 10^{-6}$ & ${ }^{6} 8.000 \times 10^{-5}$ & $1.126 \times 10^{-8}$ & $1.659 \times 10^{-7}$ & $8.813 \times 10^{-6}$ & $2.332 \times 10^{-4}$ & $8.658 \times 10^{-7}$ \\
\hline 6.00 & $1.699 \times 10^{-4}$ & $1.288 \times 10^{-4}$ & $9.651 \times 10^{-5}$ & $3.808 \times 10^{-5}$ & $1.056 \times 10^{-5}$ & $58.725 \times 10^{-5}$ & $5 \quad 0.0$ & $1.448 \times 10^{-7}$ & $9.480 \times 10^{-6}$ & $2.357 \times 10^{-4}$ & $1.196 \times 10^{-6}$ \\
\hline 6.50 & $1.841 \times 10^{-4}$ & $1.383 \times 10^{-4}$ & $1.064 \times 10^{-4}$ & $4.086 \times 10^{-5}$ & $1.119 \times 10^{-5}$ & $58.809 \times 10^{-5}$ & $1.444 \times 10^{-7}$ & $2.065 \times 10^{-6}$ & $1.063 \times 10^{-5}$ & $2.427 \times 10^{-4}$ & $4.977 \times 10^{-6}$ \\
\hline 7.00 & $1.983 \times 10^{-4}$ & $1.477 \times 10^{-4}$ & $1.168 \times 10^{-4}$ & $4.377 \times 10^{-5}$ & $1.180 \times 10^{-5}$ & $58.890 \times 10^{-5}$ & $1.679 \times 10^{-7}$ & $2.906 \times 10^{-6}$ & $1.062 \times 10^{-5}$ & $2.441 \times 10^{-4}$ & $1.354 \times 10^{-6}$ \\
\hline
\end{tabular}


Table A.1. continued.

\begin{tabular}{|c|c|c|c|c|c|c|c|c|c|c|c|}
\hline \multirow[b]{2}{*}{$M_{*}$} & \multicolumn{6}{|c|}{ M stars } & \multicolumn{3}{|c|}{ S stars } & \multicolumn{2}{|l|}{ C stars } \\
\hline & forsterite & fayalite & enstatite & ferrosilite & quartz & iron & quartz & iron & $\mathrm{SiC}$ & carbon & iron \\
\hline \multicolumn{12}{|c|}{$Z=0.015$} \\
\hline 1.00 & $4.972 \times 10^{-5}$ & $3.838 \times 10^{-5}$ & $2.760 \times 10^{-5}$ & $1.082 \times 10^{-5}$ & $3.122 \times 10^{-6}$ & $2.627 \times 10^{-5}$ & 0.0 & 0.0 & 0.0 & 0.0 & 0.0 \\
\hline 1.10 & $7.186 \times 10^{-5}$ & $5.531 \times 10^{-5}$ & $4.116 \times 10^{-5}$ & $1.576 \times 10^{-5}$ & $4.414 \times 10^{-6}$ & $3.490 \times 10^{-5}$ & 0.0 & 0.0 & 0.0 & 0.0 & 0.0 \\
\hline 1.20 & $7.830 \times 10^{-5}$ & $6.048 \times 10^{-5}$ & $4.529 \times 10^{-5}$ & $1.742 \times 10^{-5}$ & $4.795 \times 10^{-6}$ & $3.778 \times 10^{-5}$ & 0.0 & 0.0 & 0.0 & 0.0 & 0.0 \\
\hline 1.25 & $8.571 \times 10^{-5}$ & $6.600 \times 10^{-5}$ & $5.063 \times 10^{-5}$ & $1.920 \times 10^{-5}$ & $5.182 \times 10^{-6}$ & $3.795 \times 10^{-5}$ & 0.0 & 0.0 & 0.0 & 0.0 & 0.0 \\
\hline 1.30 & $1.013 \times 10^{-4}$ & $7.811 \times 10^{-5}$ & $6.007 \times 10^{-5}$ & $2.280 \times 10^{-5}$ & $6.106 \times 10^{-6}$ & $4.413 \times 10^{-5}$ & 0.0 & 0.0 & 0.0 & 0.0 & 0.0 \\
\hline 1.40 & $1.084 \times 10^{-4}$ & $8.355 \times 10^{-5}$ & $6.393 \times 10^{-5}$ & $2.448 \times 10^{-5}$ & $6.629 \times 10^{-6}$ & $5.319 \times 10^{-5}$ & 0.0 & 0.0 & 0.0 & 0.0 & 0.0 \\
\hline 1.50 & $1.247 \times 10^{-4}$ & $9.559 \times 10^{-5}$ & $7.359 \times 10^{-5}$ & $2.804 \times 10^{-5}$ & $7.678 \times 10^{-6}$ & $6.819 \times 10^{-5}$ & 0.0 & 0.0 & $1.009 \times 10^{-5}$ & $8.351 \times 10^{-5}$ & $54.482 \times 10^{-7}$ \\
\hline 1.60 & $1.377 \times 10^{-4}$ & $1.049 \times 10^{-4}$ & $8.138 \times 10^{-5}$ & $3.094 \times 10^{-5}$ & $8.542 \times 10^{-6}$ & $8.295 \times 10^{-5}$ & 0.0 & 0.0 & $9.311 \times 10^{-7}$ & $1.147 \times 10^{-5}$ & $51.356 \times 10^{-6}$ \\
\hline 1.70 & $6.938 \times 10^{-5}$ & $3.016 \times 10^{-5}$ & $8.386 \times 10^{-5}$ & $1.832 \times 10^{-5}$ & $1.915 \times 10^{-5}$ & $2.593 \times 10^{-4}$ & 0.0 & 0.0 & $798 \times 10^{-5}$ & $2.346 \times 10^{-4}$ & $1.979 \times 10^{-7}$ \\
\hline 1.80 & $1.097 \times 10^{-5}$ & $8.462 \times 10^{-6}$ & $7.290 \times 10^{-6}$ & $2.733 \times 10^{-6}$ & $6.504 \times 10^{-7}$ & $4.265 \times 10^{-6}$ & $1.167 \times 10^{-4}$ & $3.145 \times 10^{-9}$ & $5.149 \times 10^{-5}$ & $4.325 \times 10^{-4}$ & $5.524 \times 10^{-5}$ \\
\hline 1.90 & $1.130 \times 10^{-5}$ & $8.715 \times 10^{-6}$ & $7.354 \times 10^{-6}$ & $2.783 \times 10^{-6}$ & $6.754 \times 10^{-7}$ & $4.843 \times 10^{-6}$ & 0.0 & $8.338 \times 10^{-7}$ & $9.924 \times 10^{-5}$ & $9.462 \times 10^{-4}$ & $1.389 \times 10^{-5}$ \\
\hline 2.00 & $1.143 \times 10^{-5}$ & $8.817 \times 10^{-6}$ & $7.597 \times 10^{-6}$ & $2.897 \times 10^{-6}$ & $6.928 \times 10^{-7}$ & $5.458 \times 10^{-6}$ & 0.0 & 0.0 & $2.133 \times 10^{-4}$ & $1.849 \times 10^{-3}$ & $8.450 \times 10^{-5}$ \\
\hline 2.10 & $8.374 \times 10^{-6}$ & $6.165 \times 10^{-6}$ & $6.267 \times 10^{-6}$ & $2.458 \times 10^{-6}$ & $6.379 \times 10^{-7}$ & $6.252 \times 10^{-6}$ & 0.0 & 0.0 & $2.112 \times 10^{-4}$ & $2.686 \times 10^{-3}$ & $9.150 \times 10^{-6}$ \\
\hline 2.20 & $6.073 \times 10^{-6}$ & $3.488 \times 10^{-6}$ & $5.872 \times 10^{-6}$ & $1.549 \times 10^{-6}$ & $1.066 \times 10^{-6}$ & $1.035 \times 10^{-5}$ & 0.0 & 0.0 & $2.184 \times 10^{-4}$ & $3.652 \times 10^{-3}$ & $3.750 \times 10^{-6}$ \\
\hline 2.30 & $2.114 \times 10^{-9}$ & $2.026 \times 10^{-9}$ & $1.023 \times 10^{-9}$ & 0.0 & 0.0 & $1.968 \times 10^{-9}$ & $3.523 \times 10^{-5}$ & 0.0 & $2.366 \times 10^{-4}$ & $4.655 \times 10^{-3}$ & $2.680 \times$ \\
\hline 2.40 & $1.208 \times 10^{-9}$ & $1.048 \times 10^{-9}$ & $1.113 \times 10^{-9}$ & & 0.0 & $2.327 \times 10^{-9}$ & 0.0 & & $.411 \times 10^{-4}$ & $5.677 \times 10^{-3}$ & $3.488 \times 1$ \\
\hline 2.50 & 0.0 & 0.0 & 0.0 & 0. & 0.0 & 0.0 & 0.0 & 0 . & $3^{-4}$ & $6.506 \times 10^{-3}$ & $6.127 \times 10^{-6}$ \\
\hline 3.00 & 0.0 & 0.0 & 0 & 0. & 0.0 & 0.0 & 0 & 0.0 & $.258 \times 10^{-4}$ & $1.338 \times 10^{-2}$ & $2.450 \times 10^{-7}$ \\
\hline 3.50 & 0.0 & 0.0 & 0.0 & 0.0 & 0.0 & 0.0 & 0.0 & 0.0 & $4.328 \times 10^{-4}$ & $8.747 \times 10^{-3}$ & $8.492 \times 10^{-7}$ \\
\hline 4.00 & 0.0 & 0.0 & 0.0 & 0.0 & 0.0 & $1.038 \times 10^{-9}$ & 0.0 & 0.0 & $.015 \times 10^{-4}$ & $6.385 \times 10^{-3}$ & $6.446 \times 10^{-6}$ \\
\hline 4.01 & 0.0 & 0.0 & 0.0 & 0.0 & 0.0 & $1.029 \times 10^{-9}$ & 0.0 & 0.0 & $6.030 \times 10^{-4}$ & $6.465 \times 10^{-3}$ & $6.272 \times 10^{-6}$ \\
\hline 4.50 & $5.104 \times 10^{-4}$ & $3.923 \times 10^{-4}$ & $4.112 \times 10^{-4}$ & $1.467 \times 10^{-4}$ & $2.628 \times 10^{-5}$ & $1.141 \times 10^{-4}$ & 0.0 & $3.620 \times 10^{-5}$ & $1.897 \times 10^{-5}$ & $9.433 \times 10^{-5}$ & $1.195 \times 10^{-6}$ \\
\hline 5.00 & $5.590 \times$ & $4.283 \times 10^{-4}$ & $4.723 \times 10^{-4}$ & $1.665 \times 10^{-4}$ & $2.809 \times 10^{-5}$ & $1.137 \times 10^{-4}$ & $3.314 \times 10^{-6}$ & $9.557 \times 1$ & $2.855 \times 10^{-5}$ & $8.266 \times 10^{-5}$ & $1.742 \times 1$ \\
\hline 5.50 & 6.023 & 4.596 & $5.356 \times 10^{-4}$ & $1.866 \times 10^{-4}$ & $2.972 \times 10^{-5}$ & $1.163 \times 10^{-4}$ & 0.0 & $1.836 \times$ & $2.789 \times 10^{-5}$ & $8.240 \times 10^{-5}$ & \\
\hline 6.00 & 6.412 & 4.876 & $5.970 \times 10^{-4}$ & $2.056 \times 10^{-4}$ & $3.100 \times 10^{-5}$ & $1.165 \times 10^{-4}$ & $8.729 \times 10^{-7}$ & $9.972 \times$ & & $7.776 \times 10^{-5}$ & $1.870 \times 10^{-5}$ \\
\hline 6.50 & 6.759 & $5.126 \times 10^{-4}$ & $6.566 \times 10^{-4}$ & $2.238 \times 10^{-4}$ & $3.206 \times 10^{-5}$ & $1.159 \times 10^{-4}$ & $1.395 \times 10^{-6}$ & $1.288 \times 1$ & $2.694 \times 10^{-5}$ & $8.094 \times 10^{-5}$ & $1.715 \times 10^{-5}$ \\
\hline 7.00 & $7.087 \times 10^{-4}$ & $5.357 \times 10^{-4}$ & $7.164 \times 10^{-4}$ & $2.417 \times 10^{-4}$ & $3.313 \times 10^{-5}$ & $1.166 \times 10^{-4}$ & $1.720 \times 10^{-6}$ & $1.871 \times 10^{-5}$ & $2.819 \times 10^{-5}$ & $7.782 \times 10^{-5}$ & $1.235 \times 10^{-5}$ \\
\hline \multicolumn{12}{|c|}{$Z=0.02$} \\
\hline 1.00 & $1.416 \times 10^{-4}$ & $1.103 \times 10^{-4}$ & $8.849 \times 10^{-5}$ & $3.324 \times 10^{-5}$ & $7.925 \times 10^{-6}$ & $5.077 \times 10^{-5}$ & 0.0 & 0.0 & 0.0 & 0.0 & 0.0 \\
\hline & & & $10^{-4}$ & 4.139 & $9.785 \times 10^{-6}$ & $6.522 \times 1$ & 0.0 & 0.0 & 0 & 0.0 & 0.0 \\
\hline 1.20 & $2.194 \times$ & $1.712 \times 10^{-4}$ & $1.411 \times 10^{-4}$ & $5.292 \times 10^{-5}$ & $1.209 \times 10^{-5}$ & $7.449 \times 10^{-5}$ & 0.0 & 0.0 & 0.0 & 0.0 & 0.0 \\
\hline 1.25 & $2.532 \times 10^{-4}$ & $1.979 \times 10^{-4}$ & $1.624 \times 10^{-4}$ & $6.118 \times 10^{-5}$ & $1.400 \times 10^{-5}$ & $8.641 \times 10^{-5}$ & 0.0 & 0. & 0. & 0.0 & 0.0 \\
\hline 1.30 & $2.396 \times 10^{-4}$ & $1.875 \times 10^{-4}$ & $1.554 \times 10^{-4}$ & $5.855 \times 10^{-5}$ & $1.318 \times 10^{-5}$ & $7.955 \times 10^{-5}$ & & & & 0.0 & 0.0 \\
\hline 1.40 & $2.788 \times 10^{-4}$ & $2.178 \times 10^{-4}$ & $1.849 \times 10^{-4}$ & $6.915 \times 10^{-5}$ & $1.517 \times 10^{-5}$ & $9.015 \times 10^{-5}$ & 0.0 & 0.0 & 0.0 & 0.0 & 0.0 \\
\hline 1.50 & $3.214 \times 10^{-4}$ & $2.523 \times 10^{-4}$ & $2.115 \times 10^{-4}$ & $8.031 \times 10^{-5}$ & $1.770 \times 10^{-5}$ & $1.144 \times 10^{-4}$ & 0.0 & 0.0 & 0.0 & 0.0 & 0.0 \\
\hline 1.60 & $3.297 \times 10^{-4}$ & $2.584 \times 10^{-4}$ & $2.198 \times 10^{-4}$ & $8.282 \times 10^{-5}$ & $1.802 \times 10^{-5}$ & $1.093 \times 10^{-4}$ & $9.297 \times 10^{-6}$ & $1.678 \times 10^{-6}$ & 0.0 & 0.0 & 0.0 \\
\hline 1.70 & $3.470 \times 10^{-4}$ & $2.712 \times 10^{-4}$ & $2.338 \times 10^{-4}$ & $8.786 \times 10^{-5}$ & $1.908 \times 10^{-5}$ & $1.276 \times 10^{-4}$ & 0.0 & 0.0 & $1.673 \times 10^{-5}$ & $1.200 \times 10^{-4}$ & $2.483 \times 10^{-7}$ \\
\hline 1.80 & $3.659 \times 10^{-4}$ & $2.850 \times 10^{-4}$ & $2.506 \times 10^{-4}$ & $9.347 \times 10^{-5}$ & $2.015 \times 10^{-5}$ & $1.387 \times 10^{-4}$ & 0. & 0 . & $397 \times 10^{-5}$ & $5.696 \times 10^{-5}$ & $3.038 \times 10^{-5}$ \\
\hline 1.90 & $3.516 \times 10^{-4}$ & 2.500 & $\times 10^{-4}$ & $1.118 \times 10^{-4}$ & $2.626 \times 10^{-5}$ & $2.586 \times 10^{-4}$ & 0.0 & 0.0 & $3.244 \times 10^{-5}$ & $2.813 \times 10^{-4}$ & $3.029 \times 10^{-7}$ \\
\hline 2.00 & $2.427 \times 10^{-4}$ & $1.765 \times 10^{-4}$ & $1.882 \times 10^{-4}$ & $7.070 \times 10^{-5}$ & $1.621 \times 10^{-5}$ & $1.681 \times 10^{-4}$ & 0.0 & 0.0 & $2.934 \times 10^{-5}$ & $4.076 \times 10^{-4}$ & $1.361 \times 10^{-4}$ \\
\hline 2.10 & $3.251 \times 10^{-5}$ & $2.440 \times 10^{-5}$ & $3.027 \times 10^{-5}$ & $1.111 \times 10^{-5}$ & $2.169 \times 10^{-6}$ & $1.533 \times 10^{-5}$ & 0.0 & $7.873 \times 10^{-5}$ & $2.168 \times 10^{-4}$ & $8.662 \times 10^{-4}$ & $2.598 \times 10^{-4}$ \\
\hline 2.20 & $2.498 \times 10^{-5}$ & $1.978 \times 10^{-5}$ & $1.888 \times 10^{-5}$ & $7.149 \times 10^{-6}$ & $1.366 \times 10^{-6}$ & $8.374 \times 10^{-6}$ & $8.014 \times 10^{-5}$ & $4.558 \times 10^{-6}$ & $2.580 \times 10^{-4}$ & $1.393 \times 10^{-3}$ & $1.314 \times 10^{-4}$ \\
\hline 2.30 & $7.373 \times 10^{-6}$ & $5.596 \times 10^{-6}$ & $4.535 \times 10^{-6}$ & $1.670 \times 10^{-6}$ & $4.370 \times 10^{-7}$ & $3.462 \times 10^{-6}$ & $5.662 \times 10^{-5}$ & 0.0 & $3.925 \times 10^{-4}$ & $2.465 \times 10^{-3}$ & $9.564 \times 10^{-5}$ \\
\hline 2.40 & $2.238 \times 10^{-9}$ & $2.086 \times 10^{-9}$ & $1.169 \times 10^{-9}$ & 0.0 & 0.0 & $1.875 \times 10^{-9}$ & $2.494 \times 10^{-5}$ & 0.0 & $6.101 \times 10^{-4}$ & $4.675 \times 10^{-3}$ & $1.478 \times 10^{-4}$ \\
\hline 2.50 & 0.0 & 0.0 & 0.0 & 0.0 & 0.0 & 0.0 & $2.647 \times 10^{-5}$ & 0.0 & $6.391 \times 10^{-4}$ & $5.490 \times 10^{-3}$ & $3.434 \times 10^{-5}$ \\
\hline 3.00 & 0.0 & 0.0 & 0. & 0. & 0.0 & 0.0 & 0.0 & 0.0 & $337 \times 10^{-4}$ & $1.210 \times 10^{-2}$ & $2.153 \times 10^{-6}$ \\
\hline 3.50 & 0.0 & & & & & $1.383 \times 10^{-9}$ & & 0.0 & $9.519 \times 10^{-4}$ & $1.091 \times 10^{-2}$ & $5.244 \times 10^{-6}$ \\
\hline 4.00 & $1.711 \times 10^{-9}$ & $1.486 \times 10^{-9}$ & $1.072 \times 10^{-9}$ & 0.0 & 0.0 & $2.688 \times$ & 0.0 & 0.0 & $0^{-3}$ & $9.796 \times 10^{-3}$ & $1.685 \times 1$ \\
\hline 4.01 & $1.717 \times 10^{-9}$ & & $1.075 \times 10^{-9}$ & 0.0 & 0.0 & $2.674 \times 10^{-9}$ & 0.0 & 0.0 & & $9.851 \times 10^{-3}$ & \\
\hline 4.50 & $8.636 \times 10^{-4}$ & $6.707 \times 10^{-4}$ & $9.145 \times 10^{-4}$ & $3.179 \times 10^{-4}$ & $3.820 \times 10^{-5}$ & $1.205 \times 10^{-4}$ & $7.352 \times 10^{-8}$ & $7.790 \times 10^{-5}$ & $2.049 \times 10^{-5}$ & $4.291 \times 10^{-5}$ & $1.323 \times 10^{-6}$ \\
\hline 5.00 & $9.340 \times 10^{-4}$ & $7.230 \times 10^{-4}$ & $1.045 \times 10^{-3}$ & $3.595 \times 10^{-4}$ & $4.067 \times 10^{-5}$ & $1.248 \times 10^{-4}$ & $2.058 \times 10^{-7}$ & $5.354 \times 10^{-5}$ & $2.458 \times 10^{-5}$ & $2.435 \times 10^{-5}$ & $6.630 \times 10^{-6}$ \\
\hline 5.50 & $9.913 \times 10^{-4}$ & $7.630 \times 10^{-4}$ & $1.174 \times 10^{-3}$ & $3.987 \times 10^{-4}$ & $4.318 \times 10^{-5}$ & $1.346 \times 10^{-4}$ & 0.0 & $3.654 \times 10^{-5}$ & $1.960 \times 10^{-5}$ & $4.102 \times 10^{-5}$ & $3.937 \times 10^{-6}$ \\
\hline 6.00 & $1.037 \times 10^{-3}$ & $7.982 \times 10^{-4}$ & $1.292 \times 10^{-3}$ & $4.363 \times 10^{-4}$ & $4.389 \times 10^{-5}$ & $1.256 \times 10^{-4}$ & $4.083 \times 10^{-6}$ & $3.305 \times 10^{-5}$ & $2.678 \times 10^{-5}$ & $3.825 \times 10^{-5}$ & $1.410 \times 10^{-5}$ \\
\hline 6.50 & $1.084 \times 10^{-3}$ & $8.313 \times 10^{-4}$ & $1.421 \times 10^{-3}$ & $4.753 \times 10^{-4}$ & $4.576 \times 10^{-5}$ & $1.313 \times 10^{-4}$ & $2.233 \times 10^{-6}$ & $2.861 \times 10^{-5}$ & $2.831 \times 10^{-5}$ & $3.600 \times 10^{-5}$ & $1.681 \times 10^{-5}$ \\
\hline 7.00 & $1.123 \times 10^{-3}$ & $8.611 \times 10^{-4}$ & $1.544 \times 10^{-3}$ & $5.135 \times 10^{-4}$ & $4.679 \times 10^{-5}$ & $1.285 \times 10^{-4}$ & $3.430 \times 10^{-6}$ & $2.150 \times 10^{-5}$ & $2.761 \times 10^{-5}$ & $3.458 \times 10^{-5}$ & $2.929 \times 10^{-5}$ \\
\hline
\end{tabular}


Table A.1. continued.

\begin{tabular}{|c|c|c|c|c|c|c|c|c|c|c|c|}
\hline \multirow[b]{2}{*}{$M_{*}$} & \multicolumn{6}{|c|}{ M stars } & \multicolumn{3}{|c|}{ S stars } & \multicolumn{2}{|l|}{ C stars } \\
\hline & forsterite & fayalite & enstatite & ferrosilite & quartz & iron & quartz & iron & $\mathrm{SiC}$ & & iron \\
\hline & & & & & & $Z=0.03$ & & & & & \\
\hline 1.00 & $4.386 \times 10^{-4}$ & $3.512 \times 10^{-4}$ & $2.646 \times 10^{-4}$ & $1.050 \times 10^{-4}$ & $2.456 \times 10^{-5}$ & $1.616 \times 10^{-4}$ & 0.0 & 0.0 & 0.0 & 0.0 & 0.0 \\
\hline 1.10 & $5.537 \times 10^{-4}$ & $4.427 \times 10^{-4}$ & $3.405 \times 10^{-4}$ & $1.340 \times 10^{-4}$ & $3.069 \times 10^{-5}$ & $1.965 \times 10^{-4}$ & 0.0 & 0.0 & 0.0 & 0.0 & 0.0 \\
\hline 1.20 & $6.244 \times 10^{-4}$ & $5.028 \times 10^{-4}$ & $3.879 \times 10^{-4}$ & $1.546 \times 10^{-4}$ & $3.450 \times 10^{-5}$ & $2.272 \times 10^{-4}$ & 0.0 & 0.0 & 0.0 & 0.0 & 0.0 \\
\hline 1.25 & $6.729 \times 10^{-4}$ & $5.407 \times 10^{-4}$ & $4.264 \times 10^{-4}$ & $1.681 \times 10^{-4}$ & $3.666 \times 10^{-5}$ & $2.280 \times 10^{-4}$ & 0.0 & 0.0 & 0.0 & 0.0 & 0.0 \\
\hline 1.30 & $7.104 \times 10^{-4}$ & $5.710 \times 10^{-4}$ & $4.574 \times 10^{-4}$ & $1.795 \times 10^{-4}$ & $3.827 \times 10^{-5}$ & $2.253 \times 10^{-4}$ & 0.0 & 0.0 & 0.0 & 0.0 & 0.0 \\
\hline 1.40 & $8.048 \times 10^{-4}$ & $6.461 \times 10^{-4}$ & $5.216 \times 10^{-4}$ & $2.036 \times 10^{-4}$ & $4.305 \times 10^{-5}$ & $2.456 \times 10^{-4}$ & 0.0 & 0.0 & 0.0 & 0.0 & 0.0 \\
\hline 1.50 & $836 \times 10^{-4}$ & $7.152 \times 10^{-4}$ & $5.850 \times 10^{-4}$ & $2.310 \times 10^{-4}$ & $4.695 \times 10^{-5}$ & $2.821 \times 10^{-4}$ & 0.0 & 0.0 & 0.0 & 0.0 & 0.0 \\
\hline 1.60 & $8.959 \times 10^{-4}$ & $7.084 \times 10^{-4}$ & $6.333 \times 10^{-4}$ & $2.353 \times 10^{-4}$ & $5.421 \times 10^{-5}$ & $3.397 \times 10^{-4}$ & 0.0 & 0.0 & 0.0 & 0.0 & 0.0 \\
\hline 1.70 & $9.869 \times 10^{-4}$ & $7.946 \times 10^{-4}$ & $6.949 \times 10^{-4}$ & $2.681 \times 10^{-4}$ & $5.053 \times 10^{-5}$ & $2.732 \times 10^{-4}$ & 0.0 & 0.0 & $3.828 \times 10^{-6}$ & $5.612 \times 10^{-5}$ & $51.336 \times 10^{-8}$ \\
\hline 1.80 & $1.060 \times 10^{-3}$ & $8.593 \times 10^{-4}$ & $7.994 \times 10^{-4}$ & $3.067 \times 10^{-4}$ & $5.214 \times 10^{-5}$ & $2.732 \times 10^{-4}$ & 0.0 & 0.0 & $.958 \times 10^{-5}$ & $1.531 \times 10^{-4}$ & ${ }^{4} 6.882 \times 10^{-6}$ \\
\hline 1.90 & $1.098 \times 10^{-3}$ & $8.890 \times 10^{-4}$ & $8.710 \times 10^{-4}$ & $3.315 \times 10^{-4}$ & $5.297 \times 10^{-5}$ & $2.758 \times 10^{-4}$ & 0.0 & 0.0 & $8.287 \times 10^{-5}$ & $1.541 \times 10^{-4}$ & ${ }^{4} 1.374 \times 10^{-4}$ \\
\hline 2.00 & $1.157 \times 10^{-3}$ & $9.315 \times 10^{-4}$ & $9.931 \times 10^{-4}$ & $3.703 \times 10^{-4}$ & $5.412 \times 10^{-5}$ & $3.117 \times 10^{-4}$ & 0.0 & 0.0 & $5.386 \times 10^{-5}$ & $3.387 \times 10^{-4}$ & ${ }^{4} 7.887 \times 10^{-7}$ \\
\hline 2.10 & $1.032 \times 10^{-3}$ & $7.188 \times 10^{-4}$ & $1.152 \times 10^{-3}$ & $3.470 \times 10^{-4}$ & $8.928 \times 10^{-5}$ & $7.246 \times 10^{-4}$ & 0.0 & 0.0 & $4.548 \times 10^{-5}$ & $4.676 \times 10^{-4}$ & $2.163 \times 10^{-7}$ \\
\hline 2.20 & $8.239 \times 10^{-4}$ & $6.437 \times 10^{-4}$ & $7.881 \times 10^{-4}$ & $2.941 \times 10^{-4}$ & $4.160 \times 10^{-5}$ & $2.908 \times 10^{-4}$ & 0.0 & $6.207 \times 10^{-4}$ & $3.795 \times 10^{-5}$ & $6.144 \times 10^{-4}$ & $1.149 \times 10^{-7}$ \\
\hline 2.30 & $1.190 \times 10^{-4}$ & $9.143 \times 10^{-5}$ & $1.050 \times 10^{-4}$ & $3.919 \times 10^{-5}$ & $6.678 \times 10^{-6}$ & $5.673 \times 10^{-5}$ & $1.950 \times 10^{-3}$ & $1.578 \times 10^{-8}$ & $1.523 \times 10^{-4}$ & $7.633 \times 10^{-4}$ & $8.779 \times 10^{-4}$ \\
\hline 2.40 & $4.101 \times 10^{-5}$ & $3.257 \times 10^{-5}$ & $4.915 \times 10^{-5}$ & $1.817 \times 10^{-5}$ & $2.000 \times 10^{-6}$ & $1.122 \times 10^{-5}$ & $1.833 \times 10^{-4}$ & $5.113 \times 10^{-4}$ & $3.347 \times 10^{-4}$ & $1.017 \times 10^{-3}$ & \\
\hline 2.50 & 2.80 & $1.425 \times 10^{-5}$ & $3.954 \times 10^{-5}$ & $7.333 \times 10^{-6}$ & $4.294 \times 10^{-6}$ & $3.770 \times 10^{-5}$ & 0.0 & $2.692 \times 10^{-5}$ & $5.993 \times$ & $2.126 \times 1$ & 9.256 \\
\hline 3.00 & $1.235 \times 10^{-9}$ & $1.077 \times 10^{-9}$ & 0.0 & 0.0 & 0.0 & $1.966 \times 10^{-9}$ & 0.0 & $2.250 \times 10^{-9}$ & $1.847 \times 10^{-3}$ & $1.087 \times 10^{-2}$ & $1.026 \times 10^{-4}$ \\
\hline 3.50 & $2.516 \times 10^{-9}$ & $2.222 \times 10^{-9}$ & $1.613 \times 10^{-9}$ & 0.0 & 0.0 & $4.438 \times 10^{-9}$ & 0.0 & $3.632 \times 10^{-9}$ & $2.133 \times 10^{-3}$ & $1.448 \times 10^{-2}$ & $5.793 \times 10^{-5}$ \\
\hline 4.00 & $3.752 \times 10^{-9}$ & $3.411 \times 10^{-9}$ & $2.276 \times 10^{-9}$ & $1.408 \times 10^{-9}$ & 0.0 & $6.132 \times 10^{-9}$ & 0.0 & $5.517 \times 10^{-9}$ & $2.549 \times 10^{-3}$ & $1.707 \times 10^{-2}$ & $5.820 \times 10^{-5}$ \\
\hline 4.01 & $3.815 \times 10^{-9}$ & $3.469 \times 10^{-9}$ & $2.300 \times 10^{-9}$ & $1.422 \times 10^{-9}$ & 0.0 & $6.122 \times 10^{-9}$ & 0.0 & $5.482 \times 10^{-9}$ & $2.550 \times 10^{-3}$ & $1.709 \times 10^{-2}$ & $5.885 \times 10^{-5}$ \\
\hline 4.50 & $1.763 \times 10^{-3}$ & $1.396 \times 10^{-3}$ & $2.622 \times 10^{-3}$ & $8.934 \times 10^{-4}$ & $6.470 \times 10^{-5}$ & $1.729 \times 10^{-4}$ & 0.0 & 0.0 & $2.459 \times 10^{-5}$ & $7.382 \times 10^{-5}$ & $4.483 \times 10^{-6}$ \\
\hline 5.00 & $1.834 \times 10^{-3}$ & $1.449 \times 10^{-3}$ & $2.984 \times 10^{-3}$ & $1.010 \times 10^{-3}$ & $6.763 \times 10^{-5}$ & $1.767 \times 10^{-4}$ & 0.0 & 0.0 & $7.030 \times 10^{-6}$ & $6.068 \times 10^{-5}$ & $3.297 \times 10^{-7}$ \\
\hline 5.50 & $1.877 \times 10^{-3}$ & $1.485 \times 10^{-3}$ & $3.318 \times 10^{-3}$ & $1.117 \times 10^{-3}$ & $6.937 \times 10^{-5}$ & $1.750 \times 10^{-4}$ & 0.0 & $1.354 \times 10^{-4}$ & 0.0 & 0.0 & 0.0 \\
\hline 6.00 & $1.934 \times 10^{-3}$ & $8 \times 10^{-3}$ & $3.668 \times 10^{-3}$ & $1.228 \times 10^{-3}$ & $7.167 \times 10^{-5}$ & $1.777 \times 10^{-4}$ & $1.242 \times 10^{-5}$ & & $1.469 \times 10^{-5}$ & $1.239 \times 10^{-5}$ & $7.681 \times 10^{-6}$ \\
\hline 6.50 & $1.987 \times 10^{-3}$ & $1.569 \times 10^{-3}$ & $4.024 \times 10^{-3}$ & $1.340 \times 10^{-3}$ & $7.397 \times 10^{-5}$ & $1.831 \times 10^{-4}$ & $7.084 \times 10^{-6}$ & $1.684 \times 10^{-5}$ & & $1.327 \times 10^{-5}$ & \\
\hline 7.00 & $2.028 \times 10^{-3}$ & $1.600 \times 10^{-3}$ & $4.381 \times 10^{-3}$ & $1.452 \times 10^{-3}$ & $7.638 \times 10^{-5}$ & $1.905 \times 10^{-4}$ & $5.351 \times 10^{-6}$ & $3.804 \times 10^{-5}$ & $1.579 \times 10^{-5}$ & $7.799 \times 10^{-6}$ & $2.605 \times 10^{-5}$ \\
\hline & & & & & & $Z=0.04$ & & & & & \\
\hline 1.00 & $7.962 \times 10^{-4}$ & $6.493 \times 10^{-4}$ & $4.946 \times 10^{-4}$ & $1.988 \times 10^{-4}$ & $4.265 \times 10^{-5}$ & $2.709 \times 10^{-4}$ & 0.0 & 0.0 & 0.0 & 0.0 & 0.0 \\
\hline 1.10 & $1.118 \times 10^{-3}$ & $9.171 \times 10^{-4}$ & $6.973 \times 10^{-4}$ & $2.841 \times 10^{-4}$ & $5.995 \times 10^{-5}$ & $3.824 \times 10^{-4}$ & 0.0 & 0.0 & 0.0 & 0.0 & .0 \\
\hline 1.20 & $1.312 \times 10^{-3}$ & $1.077 \times 10^{-3}$ & $8.322 \times 10^{-4}$ & $3.371 \times 10^{-4}$ & $6.966 \times 10^{-5}$ & $4.332 \times 10^{-4}$ & 0.0 & 0. & 0. & 0.0 & .0 \\
\hline 1.25 & $1.303 \times 10^{-3}$ & $1.072 \times 10^{-3}$ & $8.409 \times 10^{-4}$ & $3.411 \times 10^{-4}$ & $6.869 \times 10^{-5}$ & $4.324 \times 10^{-4}$ & 0.0 & 0.0 & 0.0 & 0.0 & 0.0 \\
\hline 1.30 & $1.405 \times 10^{-3}$ & $1.160 \times 10^{-3}$ & $9.183 \times 10^{-4}$ & $3.734 \times 10^{-4}$ & $7.339 \times 10^{-5}$ & $4.522 \times 10^{-4}$ & 0.0 & 0. & & 0.0 & 0.0 \\
\hline 1.40 & $1.576 \times 10^{-3}$ & $1.299 \times 10^{-3}$ & $1.062 \times 10^{-3}$ & $4.264 \times 10^{-4}$ & $8.021 \times 10^{-5}$ & $4.497 \times 10$ & 0.0 & 0. & & 0 & .0 \\
\hline 1.50 & $1.715 \times 10^{-3}$ & $1.415 \times 10^{-3}$ & $1.175 \times 10^{-3}$ & $4.698 \times 10^{-4}$ & $8.648 \times 10^{-5}$ & $4.710 \times 10^{-4}$ & 0.0 & 0. & 0 . & 0.0 & 0.0 \\
\hline 1.60 & $1.808 \times 10^{-3}$ & $1.496 \times 10^{-3}$ & $1.270 \times 10^{-3}$ & $5.077 \times 10^{-4}$ & $9.044 \times 10^{-5}$ & $5.005 \times 10^{-4}$ & 0.0 & 0. & 0.0 & 0.0 & 0.0 \\
\hline 1.70 & $2.038 \times 10^{-3}$ & $1.693 \times 10^{-3}$ & $1.447 \times 10^{-3}$ & $5.813 \times 10^{-4}$ & $1.014 \times 10^{-4}$ & $5.593 \times 10^{-4}$ & 0.0 & 0.0 & 0.0 & 0.0 & 0.0 \\
\hline 1.80 & $2.017 \times 10^{-3}$ & $1.657 \times 10^{-3}$ & $1.492 \times 10^{-3}$ & $5.833 \times 10^{-4}$ & $9.774 \times 10^{-5}$ & $4.895 \times 10^{-4}$ & 0.0 & 0.0 & 0.0 & 0.0 & 0.0 \\
\hline 1.90 & $117 \times 10^{-3}$ & $.740 \times 10^{-3}$ & $1.600 \times 10^{-3}$ & $6.237 \times 10^{-4}$ & $1.015 \times 10^{-4}$ & $4.975 \times 10^{-4}$ & 0. & 0. & 0.0 & 0.0 & 0.0 \\
\hline 2.00 & $2.371 \times 10^{-3}$ & $1.959 \times 10^{-3}$ & $1.788 \times 10^{-3}$ & $7.046 \times 10^{-4}$ & $1.146 \times 10^{-4}$ & $5.901 \times 10^{-4}$ & 0.0 & 0.0 & 0.0 & 0.0 & 0.0 \\
\hline 2.10 & $2.129 \times 10^{-3}$ & $1.703 \times 10^{-3}$ & $1.981 \times 10^{-3}$ & $7.579 \times 10^{-4}$ & $1.047 \times 10^{-4}$ & $5.523 \times 10^{-4}$ & 0.0 & 0.0 & $4.692 \times 10^{-5}$ & $4.709 \times 10^{-4}$ & $4.094 \times 10^{-7}$ \\
\hline 2.20 & $1.984 \times 10^{-3}$ & $1.644 \times 10^{-3}$ & $2.021 \times 10^{-3}$ & $7.606 \times 10^{-4}$ & $8.520 \times 10^{-5}$ & $4.344 \times 10^{-4}$ & 0. & $3.409 \times 10^{-4}$ & $1.256 \times 10^{-5}$ & $3.706 \times 10^{-4}$ & $2.403 \times 10^{-8}$ \\
\hline 2.30 & $1.608 \times 10^{-3}$ & $1.183 \times 10^{-3}$ & $2.429 \times 10^{-3}$ & $8.830 \times 10^{-4}$ & $8.978 \times 10^{-5}$ & $5.910 \times 10^{-4}$ & 0.0 & 0.0 & $1.390 \times 10^{-4}$ & $6.324 \times 10^{-4}$ & $5.151 \times 10^{-4}$ \\
\hline 2.40 & $4.971 \times 10^{-4}$ & $3.744 \times 10^{-4}$ & $6.920 \times 10^{-4}$ & $2.600 \times 10^{-4}$ & $2.753 \times 10^{-5}$ & $2.087 \times 10^{-4}$ & $3.967 \times 10^{-3}$ & $4.553 \times 10^{-8}$ & $5.922 \times 10^{-5}$ & $8.619 \times 10^{-4}$ & $1.399 \times 10^{-3}$ \\
\hline 2.50 & $5.073 \times 10^{-5}$ & $3.269 \times 10^{-5}$ & $1.064 \times 10^{-4}$ & $3.147 \times 10^{-5}$ & $3.924 \times 10^{-6}$ & $3.041 \times 10^{-5}$ & $1.851 \times 10^{-3}$ & $2.419 \times 10^{-3}$ & $2.513 \times 10^{-4}$ & $7.544 \times 10^{-4}$ & $3.122 \times 10^{-5}$ \\
\hline 3.00 & $5.884 \times 10^{-9}$ & $5.412 \times 10^{-9}$ & $3.584 \times 10^{-9}$ & $2.293 \times 10^{-9}$ & 0.0 & $9.373 \times 10^{-9}$ & 0.0 & $2.536 \times 10^{-8}$ & $2.981 \times 10^{-3}$ & $9.306 \times 10^{-3}$ & $2.308 \times 10^{-3}$ \\
\hline 3.50 & $5.893 \times 10^{-9}$ & $5.374 \times 10^{-9}$ & $3.850 \times 10^{-9}$ & $2.458 \times 10^{-9}$ & 0.0 & $1.178 \times 10^{-8}$ & 0.0 & $1.908 \times 10^{-8}$ & $3.484 \times 10^{-3}$ & $1.489 \times 10^{-2}$ & $4.698 \times 10^{-4}$ \\
\hline 4.00 & $9.763 \times 10^{-9}$ & $9.002 \times 10^{-9}$ & $6.214 \times 10^{-9}$ & $4.020 \times 10^{-9}$ & $1.484 \times 10^{-9}$ & $2.010 \times 10^{-8}$ & 0.0 & $2.990 \times 10^{-8}$ & $4.130 \times 10^{-3}$ & $1.816 \times 10^{-2}$ & $3.550 \times 10^{-4}$ \\
\hline 4.01 & $9.811 \times 10^{-9}$ & $9.057 \times 10^{-9}$ & $6.223 \times 10^{-9}$ & $4.032 \times 10^{-9}$ & $1.482 \times 10^{-9}$ & $2.007 \times 10^{-8}$ & 0.0 & $2.968 \times 10^{-8}$ & $4.115 \times 1$ & $1.786 \times 10^{-2}$ & $3.624 \times 10^{-4}$ \\
\hline 4.50 & $3.075 \times 10^{-3}$ & $2.464 \times 10^{-3}$ & $4.976 \times 10^{-3}$ & $1.700 \times 10^{-3}$ & $1.202 \times 10^{-4}$ & $3.029 \times 1$ & 0.0 & 0.0 & $7.947 \times 10^{-6}$ & $1.619 \times 10^{-4}$ & $3.310 \times 10^{-7}$ \\
\hline 5.00 & $3.063 \times 10^{-3}$ & $2.453 \times 10^{-3}$ & $5.778 \times 10^{-3}$ & $1.957 \times 10^{-3}$ & $1.116 \times 10^{-4}$ & $3.233 \times 10^{-4}$ & 0.0 & 0.0 & 0.0 & 0.0 & 0.0 \\
\hline 5.50 & $2.915 \times 10^{-3}$ & $2.338 \times 10^{-3}$ & $6.647 \times 10^{-3}$ & $2.234 \times 10^{-3}$ & $1.086 \times 10^{-4}$ & $3.551 \times 10^{-4}$ & 0.0 & 0.0 & $8.865 \times 10^{-6}$ & $3.758 \times 10^{-5}$ & $1.632 \times 10^{-5}$ \\
\hline 6.00 & $2.853 \times 10^{-3}$ & $2.290 \times 10^{-3}$ & $7.441 \times 10^{-3}$ & $2.485 \times 10^{-3}$ & $1.119 \times 10^{-4}$ & $3.858 \times 10^{-4}$ & 0.0 & 0.0 & $1.748 \times 10^{-5}$ & $5.776 \times 10^{-6}$ & $7.898 \times 10^{-5}$ \\
\hline 6.50 & $2.903 \times 10^{-3}$ & $2.340 \times 10^{-3}$ & $8.115 \times 10^{-3}$ & $2.707 \times 10^{-3}$ & $1.129 \times 10^{-4}$ & $3.731 \times 10^{-4}$ & $2.058 \times 10^{-5}$ & $3.143 \times 10^{-5}$ & $3.243 \times 10^{-6}$ & $1.354 \times 10^{-5}$ & $9.046 \times 10^{-6}$ \\
\hline 7.00 & $2.940 \times 10^{-3}$ & $2.377 \times 10^{-3}$ & $8.838 \times 10^{-3}$ & $2.941 \times 10^{-3}$ & $1.160 \times 10^{-4}$ & $3.678 \times 10^{-4}$ & $1.150 \times 10^{-5}$ & $9.800 \times 10^{-5}$ & 0.0 & 0.0 & 0.0 \\
\hline
\end{tabular}

